\title{
Usnic Acid Enaminone-Coupled 1,2,3-Triazoles as Antibacterial and
}

\section{Antitubercular Agents}

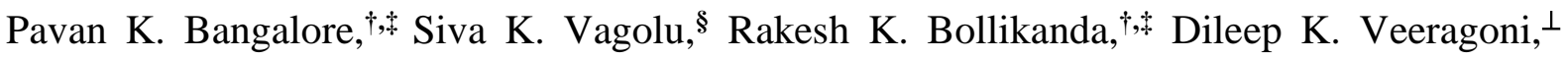

Pallavi C. Choudante, ${ }^{\perp}$ Sunil Misra, ${ }^{\perp}$ Dharmarajan Sriram, ${ }^{\S}$ Balasubramanian Sridhar, ${ }^{\|}$and Srinivas Kantevari*†

${ }^{\dagger}$ Fluoro \& Agrochemicals Division, ${ }^{\star}$ Academy of Scientific and Innovative Research (AcSIR),

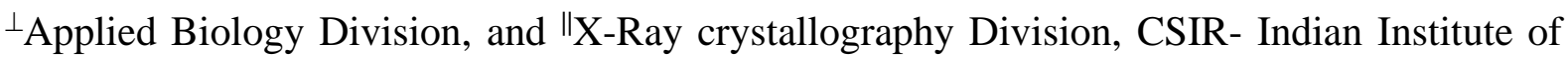
Chemical Technology, Hyderabad-500007, Telangana, India.

${ }^{\S}$ Medicinal Chemistry and Antimycobacterial Research Laboratory, Pharmacy Group, Birla Institute of Technology \& Science-Pilani, Hyderabad Campus, Jawahar Nagar, Hyderabad-500078, Telangana, India.

\section{Table of contents}

\begin{tabular}{|c|c|}
\hline \multicolumn{2}{|l|}{ CHEMISTRY } \\
\hline Scheme 1: Synthesis of $N$-Propargyl Usnic Acid Enaminone 8 & $\mathbf{S 3}$ \\
\hline X-ray crystallographic analysis of compound $\mathbf{8}$ & S4 \\
\hline Scheme 2: Synthesis of Aryl Azides A1-A26 & S5 \\
\hline Table S1: Aryl azides A1- A26 & S6-7 \\
\hline Scheme 3: Synthesis of Saccharin Azide A27 & S9 \\
\hline Scheme 4: Synthesis of Piperazine-Azides A28- A35 & S10 \\
\hline Table S2: Piperazine Azides - List of Substitutions, Codes and Yields & S11 \\
\hline Scheme 5: Synthesis of Usnic Acid Enaminone-coupled triazoles 10-44 & S13 \\
\hline Spectral data of compounds $\mathbf{1 0 - 4 4}$ & S13-36 \\
\hline${ }^{1} \mathrm{H} \&{ }^{13} \mathrm{C}$ NMR copies of New Azides, and Compounds 8, 10-44 & $\mathbf{S 4 2 - 9 5}$ \\
\hline \multicolumn{2}{|l|}{ BIOLOGY } \\
\hline In vitro $M t b$ MABA assay & S36 \\
\hline Nutrient Starvation Model & S37 \\
\hline In Vitro Antibacterial Activity and Results (Table S3) & S37-39 \\
\hline Determination of Minimum Inhibitory Concentration and Results (Table S4) & S39-40 \\
\hline In Vitro Cytotoxicity Evaluation & $\mathbf{S 4 0}$ \\
\hline Molecular Docking & S41 \\
\hline REFERENCES & S96-97 \\
\hline
\end{tabular}




\section{CHEMISTRY}

Melting points were measured by CINTEX programmable digital melting point apparatus and are reported uncorrected.Infra Red spectroscopy was performed on Thermo Nicolet nexus-670 spectrometer with reference to $\mathrm{KBr}$. Thin Layer Chromatography was performed on Merck 60 F-254 silica gel plates.NMR spectra were recorded on Avance spectrometers at 300, 400 and $500 \mathrm{MHz}$, respectively. Experiments were recorded in $\mathrm{CDCl}_{3}$ or DMSO-d $\mathrm{d}_{6}$ at $25^{\circ} \mathrm{C}$. Chemical shifts are given in parts per million (ppm) downfield from internal standard $\mathrm{Me}_{4} \mathrm{Si}$. Highresolution mass spectrometry (HRMS) [electrospray ionization (ESI)] were recorded on a highresolution QSTAR XL hybrid ms/ms system (Applied Bio systems/MDS sciex, foster city, USA), equipped with an ESI source (IICT, Hyderabad, India). Unless otherwise stated, all reagents and solvents were purchased from commercial suppliers and used without further purification. Reactions that required the use of anhydrous, inert atmosphere techniques were carried out under an atmosphere of nitrogen. Analytical high-performance liquid chromatography (HPLC) analyses were performed on a Agilent Technologies 1200 Infinity Series (1260 Infinity) using a Phenomenex C18 column $(4.6 \mathrm{~mm} \times 250 \mathrm{~mm}, 5 \mu)$ with an isocratic elution of water/acetonitrile $(20: 80 \mathrm{v} / \mathrm{v} \%)$ for $30 \mathrm{~min}$ at a flow rate of $0.5 \mathrm{~mL} / \mathrm{min}$, at $250 \mathrm{~nm}$. A purity of $>95 \%$ has been established for all tested compounds. 
Scheme 1. Synthesis of N-Propargyl Usnic Acid Enaminone 8

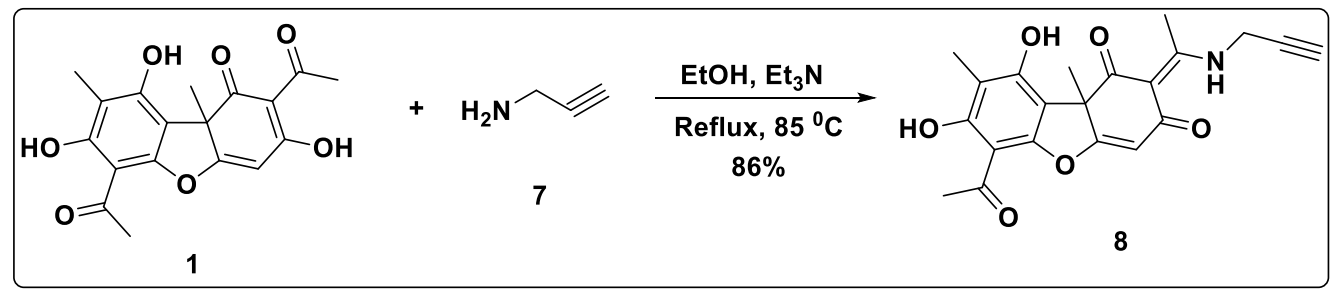

(E)-6-acetyl-7,9-dihydroxy-8,9b-dimethyl-2-(1-(prop-2-yn-1-ylamino)ethylidene)dibenzo

$[\boldsymbol{b}, \boldsymbol{d}]$ furan-1,3(2H,9bH)-dione $(\mathbf{8})$ : In a clean oven dried 500mL round bottomed flask, usnic

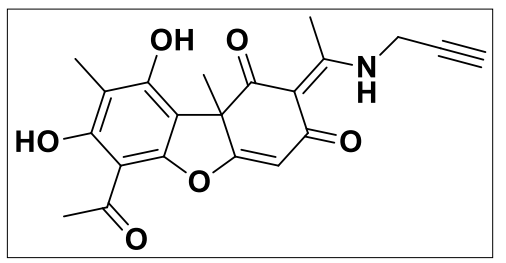

acid $(\mathbf{1}, 10.0 \mathrm{~g}, 0.03$ moles) was suspended in absolute ethanol $(200 \mathrm{~mL})$ to which triethylamine $(8.3 \mathrm{~mL}, 0.06$ moles $)$ was added and refluxed on a preheated oil bath $\left(85^{\circ} \mathrm{C}\right)$ for $5-10$ mins. Propargylamine (7) (2.0 g, 0.036 moles) was added slowly to the reaction while stirring and the contents were further refluxed for $4 \mathrm{~h}$. Following the completion of reaction, checked on TLC, the solvent was evaporated under reduced pressure and contents were extracted into ethylacetate $(100 \mathrm{~mL}$ x 3). The combined organic layers were washed with brine solution, organic layer collected and passed over a bed of sodium sulphate and evaporated under reduced pressure to give crude product. The crude was then purified by column chromatography using n-hexane-ethylacetate (7:3), to yield the desired product $\mathbf{8}$ as light yellow solid. Yield: $11.46 \mathrm{~g}$ (86.0\%); M.p.: 156-158 ${ }^{\circ} \mathrm{C}$; IR spectrum, $\left(v, \mathrm{~cm}^{-1}\right)$ : 3424.23, 3277.87, 3072.05, 2926.03, $2698.92,1691.59,1617.92,1549.21,1463.09,1417.66,1374.05,1279.68,1188.27,1135.70$, 1065.85; ${ }^{1}$ H NMR (300 MHz, CDCl $): \delta 13.68$ (s, 1H), 13.34 (s, 1H), 11.80 (s, 1H), 5.80 (s, 1H), $4.23(\mathrm{~d}, J=2.9 \mathrm{~Hz}, 2 \mathrm{H}), 2.69$ (s, 3H), $2.66(\mathrm{~s}, 3 \mathrm{H}), 2.44(\mathrm{~s}, 1 \mathrm{H}), 2.08$ (s, 3H), $1.70(\mathrm{~s}, 3 \mathrm{H})$; ${ }^{13}$ C NMR (126 MHz, CDCl3): $\delta$ 200.78, 198.78, 190.89, 175.64, 174.52, 163.62, 158.28, $155.90,108.20,105.04,102.69,102.36,101.48,76.50,74.33,57.59,33.34,31.95,31.40$, 18.48, 7.61; ESI-MS: m/z $382.20[\mathrm{M}+\mathrm{H}]^{+}$; HRMS $\left(\mathrm{C}_{21} \mathrm{H}_{19} \mathrm{NO}_{6}\right)$ ESI m/z: calcd: 382.1292 $[\mathrm{M}+\mathrm{H}]^{+}$, found: 382.1285; HPLC Rt: 16.078 min. 
X-ray Crystallography. X-ray data for the compound KA812_0m was collected at room temperature on a Bruker D8 QUEST instrument with an I $\mu$ S Mo microsource $(\lambda=0.7107 \mathrm{~A})$ and a PHOTON-100 detector. The raw data frames were reduced and corrected for absorption effects using the Bruker Apex 3 software suite programs. ${ }^{\text {a }}$ The structure was solved using intrinsic phasing method and further refined with the SHELXL ${ }^{1 b}$ program and expanded using Fourier techniques. Anisotropic displacement parameters were included for all non-hydrogen atoms. The $\mathrm{N}$-bound $\mathrm{H}$ atom and $\mathrm{O}$ bound $\mathrm{H}$ atoms were located in difference Fourier maps, and their positions and isotropic displacement parameters were refined. All $\mathrm{C}$ bound $\mathrm{H}$ atoms were positioned geometrically and treated as riding on their parent $\mathrm{C}$ atoms $[\mathrm{C}-\mathrm{H}=0.93-0.97$ $\AA$, and $\left.\mathrm{U}_{\mathrm{iso}}(\mathrm{H})=1.2 \mathrm{U}_{\text {eq }}(\mathrm{C})\right]$. In the absence of significant anomalous dispersion effects, Friedel pairs were merged. The absolute configuration has been assigned by reference to an unchanging chiral centre in the synthetic procedure.

\section{Crystal Structure Determination of Compound 8}

Crystal Data for $\mathrm{C}_{21} \mathrm{H}_{19} \mathrm{NO}_{6}(M=381.37 \mathrm{~g} / \mathrm{mol})$ : orthorhombic, space group $\mathrm{P} 2{ }_{1} 2_{1} 2_{1}$ (no. 19), $a=6.89532(9) \AA, b=12.63419(15) \AA, c=20.7068(2) \AA, V=1803.91(4) \AA^{3}, Z=4, T=$ 294.15 K, $\mu(\mathrm{MoK} \alpha)=0.104 \mathrm{~mm}^{-1}$, Dcalc $=1.404 \mathrm{~g} / \mathrm{cm}^{3}, 21931$ reflections measured $\left(5.086^{\circ}\right.$ $\left.\leq 2 \Theta \leq 61.182^{\circ}\right), 5516$ unique $\left(R_{\text {int }}=0.0322, \mathrm{R}_{\text {sigma }}=0.0302\right)$ which were used in all calculations. The final $R_{1}$ was 0.0414 (I $\left.>2 \sigma(\mathrm{I})\right)$ and $w R_{2}$ was 0.1128 (all data). CCDC 1944080 contains supplementary Crystallographic data for the structure. These data can be obtained free of charge at www.ccdc.cam.ac.uk/conts/retrieving.html [or from the Cambridge Crystallographic Data Centre (CCDC), 12 Union Road, Cambridge CB2 1EZ, UK; fax: +44(0) 1223336 033; email: deposit@ccdc.cam.ac.uk]. 
Synthesis of Azides A1-A35. The acyl bromides required for the synthesis of Azides A1-A5 and A12-A16 and A22 were prepared according to the procedure described in Method ib. Acyl bromides required for the synthesis of azides A6-A11, A17-A21 and A23-A24 were prepared by utilizing the procedure described in method ia. Similarly, acyl bromides required to prepare azides A25-A26 were synthesized according to method ic.

Scheme 2. Synthesis of Aryl Azides A1-A26

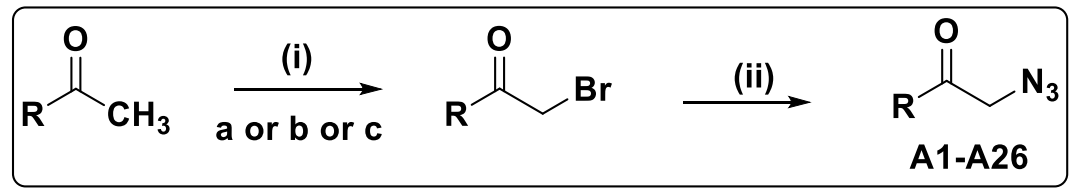

Reagents and Conditions: (i) (a) Oxone, $\mathrm{NH}_{4} \mathrm{Br}, \mathrm{MeOH}$, 1h, reflux; (b) N-bromosuccinimide, p-TSA, $\mathrm{MeOH}$, reflux, 6-10 h; (c) $\mathrm{CuBr}_{2}$, EtOAc: $\mathrm{CHCl}_{3}$ (1:1), rt, overnight; (ii) $\mathrm{NaN}_{3}$, Acetone:Ice (1:1), rt, overnight.

\section{General Procedure for Synthesis of Acyl bromides}

Method ia: A clean oven dried round bottom flask was charged with corresponding acetophenone (1.0 g, 1 eq), Oxone (1.5 eq) and ammonium bromide (1.2 eq) in methanol. The contents were refluxed for approximately one hour or till the complete conversion of acetophenone to acyl bromide as indicated on TLC. After completion of reaction, methanol was evaporated under reduced pressure and water was added to the crude mixture. The contents were extracted into chloroform (50 mL x 3), dried over sodium sulphate, evaporated under reduced pressure to get the crude product. The crude was used directly for the subsequent reaction without further purification.

Method ib: To a clean oven dried round bottom flask, corresponding acetophenone $(1.0 \mathrm{~g}, 1$ eq) and N-bromosuccinimide (1.1 eq) and $p$-toluenesulfonic acid in methanol was charged and the contents were refluxed for approximately $6-10 \mathrm{~h}$ for the complete conversion of the starting material as indicated on TLC. After completion of reaction, methanol was evaporated under reduced pressure and water was added to the crude mixture. The contents were extracted into 
chloroform or dichloromethane (50 mL x 3), dried over sodium sulphate, evaporated under reduced pressure to get the crude product. The crude was used for the next step without purification.

Method ic: In a clean dried round bottom flask, acetophenone (1.0 g, 1 equiv) was charged with copper(II)bromide (1.5 equiv) in ethylacetate-chloroform mixture (10 mL, 1:1 v/v\%) and stirred at ambient temperature overnight. The contents were filtered through a pad of celite and the solvent collected was removed under reduced pressure. The crude acyl bromides thus obtained were used as such for the synthesis of azides.

General Procedure for Synthesis of Aryl Azides A1-A26. The crude acyl bromides prepared through methods ia-c (Scheme 2) were dissolved in acetone $(10 \mathrm{~mL})$ and to it ice was added and cooled to $0{ }^{\circ} \mathrm{C}$. Sodium azide ( $3 \mathrm{eq}$ ) was added slowly, portion wise, while vigorously stirring the reaction mixture. The contents were left to stir overnight for the complete conversion of acylbromide to azide. After completion of reaction, solvent was evaporated under reduced pressure and dichloromethane or chloroform was added to the reaction mixture. The contents were partitioned between water and organic layers; organic layer collected and dried over $\mathrm{Na}_{2} \mathrm{SO}_{4}$ bed. The solvent was evaporated under reduced pressure and the product was purified using column chromatography using $n$-hexane and ethylacetate as eluent. The spectral data for few azides are in consistent with those that are available in the literature ${ }^{2-8}$ and are not given here. Data for all new azides is listed.

Table S1. Aryl Azides A1-A26

\begin{tabular}{|c|c|c|c|c|c|}
\hline Code & R/Ar- & $\begin{array}{c}\text { Overall Yield } \\
(\%)\end{array}$ & Code & R/Ar- & $\begin{array}{c}\text { Overall Yield } \\
(\%)\end{array}$ \\
\hline A1 & & 86 & A14 & & 75 \\
\hline A2 & & 83 & A15 & & 62 \\
\hline
\end{tabular}




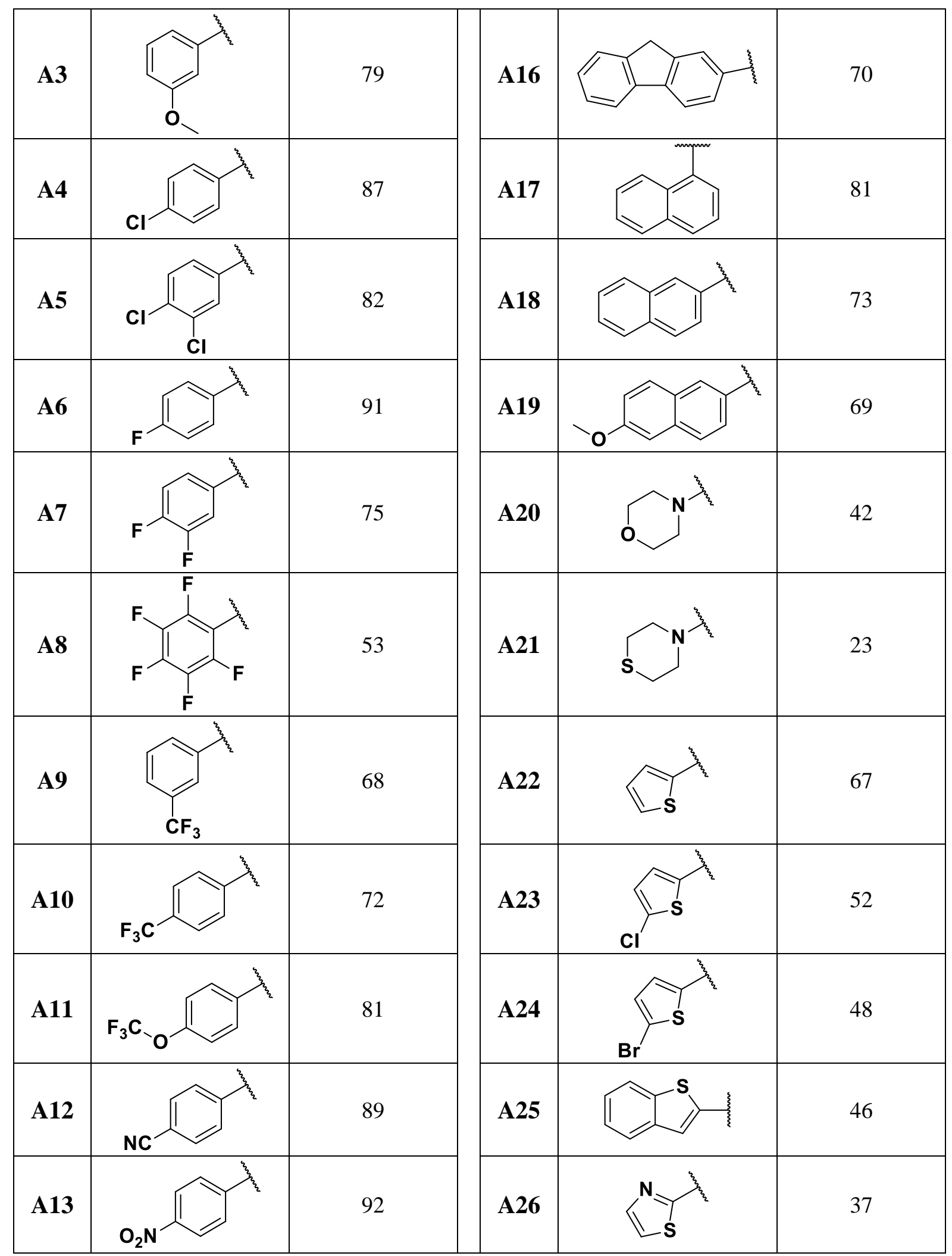


2-Azido-1-(3,4-dichlorophenyl)ethanone (A5): ${ }^{1} \mathrm{HNMR}\left(500 \mathrm{MHz}, \mathrm{CDCl}_{3}\right): \delta 8.00$ (d, $J=2.0 \mathrm{~Hz}, 1 \mathrm{H}), 7.74\left(\mathrm{dt}, J=5.6 \& 3.8 \mathrm{~Hz}, 1 \mathrm{H}, 7.61-7.57(\mathrm{~m}, 1 \mathrm{H}), 4.51(\mathrm{~s}, 2 \mathrm{H}) ;{ }^{13} \mathbf{C} \mathbf{N M R}\right.$ (126MHz, CDCl3): $\delta$ 191.38, 139.05, 134.00, 13.95, 131.29, 130.11, 127.04, 55.02.

2-Azido-1-(3,4-difluorophenyl)ethanone (A7): ${ }^{1} \mathrm{H}$ NMR (400 MHz, CDCl3): $\delta 7.78$ (ddd, $J=10.0,7.6 \& 2.1 \mathrm{~Hz}, 1 \mathrm{H}), 7.69$ (dddd, $J=8.5,4.1,2.1 \& 1.4 \mathrm{~Hz}, 1 \mathrm{H}), 7.34-7.26(\mathrm{~m}, 1 \mathrm{H}), 4.51$ (s, 2H); ${ }^{13} \mathbf{C}$ NMR (101MHz, CDCl3): $\delta$ 191.03, 153.02, 149.48, 131.53, 125.15, 118.09, $117.53,54.93$.

2-Azido-1-(3-(trifluoromethyl)phenyl)ethanone (A9): ${ }^{1} \mathrm{H}$ NMR (400 $\left.\mathrm{MHz}, \mathrm{CDCl}_{3}\right): \delta 8.16$ (s, 1H), $8.09(\mathrm{~d}, J=7.9 \mathrm{~Hz}, 1 \mathrm{H}), 7.88(\mathrm{~d}, J=7.8 \mathrm{~Hz}, 1 \mathrm{H}), 7.67(\mathrm{dd}, J=9.9 \& 5.7 \mathrm{~Hz}, 1 \mathrm{H}), 4.61(\mathrm{~s}$, 2H); ${ }^{13}$ C NMR (101MHz, CDCl3): $\delta$ 192.33, 134.95, 131.18, 130.62, 129.85, 124.91, 55.08. 1-(Anthracene-9-yl)2-azidoethanone (A15): ${ }^{1} \mathrm{H}$ NMR (400 MHz, CDCl3): $\delta 8.58$ (ddd, $J=8.9,5.0 \& 1.9 \mathrm{~Hz}, 3 \mathrm{H}), 7.86-7.77(\mathrm{~m}, 2 \mathrm{H}), 7.67-7.60(\mathrm{~m}, 3 \mathrm{H}), 7.59-7.53(\mathrm{~m}, 1 \mathrm{H}), 2.81(\mathrm{~s}$, 2H); ${ }^{13}$ C NMR (101MHz, CDCl 3$): \delta 207.64,137.42,134.24,133.64,131.19,130.22,128.78$, $128.61,128.42,127.60,127.44,127.35,124.14,124.82,123.67,34.15$.

2-Azido-1-(9H-fluoren-2-yl)ethanone (A16): ${ }^{1} \mathrm{H}$ NMR (300 MHz, CDCl 3$): \delta 8.03$ (s, 1H), $7.88(\mathrm{~d}, J=8.1 \mathrm{~Hz}, 1 \mathrm{H}), 7.81(\mathrm{dd}, J=10.3 \& 5.0 \mathrm{~Hz}, 2 \mathrm{H}), 7.61-7.55(\mathrm{~m}, 1 \mathrm{H}), 7.47-7.34(\mathrm{~m}, 2 \mathrm{H})$, $4.56(\mathrm{~s}, 2 \mathrm{H}), 3.90(\mathrm{~s}, 2 \mathrm{H}) ;{ }^{13} \mathbf{C}$ NMR (126MHz, CDCl3): $\delta$ 192.95, 147.50, 144.62, 143.67, $140.21,132.68,128.53,127.28,125.41,124.64,121.14,120.05,55.02,36.95$.

2-Azido-1-thiomorpholinoethanone (A21): ${ }^{1} \mathrm{H}$ NMR (400 MHz, CDCl3): $\delta 3.88$ (dd, $J=13.4$ \& 7.3 Hz, 4H), $3.60(\mathrm{~s}, 2 \mathrm{H}), 2.61(\mathrm{dd}, J=6.0 \& 4.0 \mathrm{~Hz}, 4 \mathrm{H}) ;{ }^{13} \mathbf{C}$ NMR $\left(\mathbf{1 0 1 M H z}, \mathbf{C D C l}_{3}\right): \delta$ $165.44,50.55,47.57,44.45,27.55,27.06$.

2-Azido-1-(5-chlorothiophen-2-yl)ethanone (A23): ${ }^{1} \mathrm{H}$ NMR (500 MHz, $\left.\mathbf{C D C l}_{3}\right): \delta 7.52(\mathrm{~d}$, $J=4.1 \mathrm{~Hz}, 1 \mathrm{H}), 6.99(\mathrm{~d}, J=4.1 \mathrm{~Hz}, 1 \mathrm{H}), 4.38(\mathrm{~s}, 2 \mathrm{H}) ;{ }^{13} \mathbf{C} \mathbf{N M R}\left(\mathbf{1 0 1 M H z}, \mathbf{C D C l}_{3}\right): \delta 185.57$, $141.40,139.40,132.32,128.00,54.55$. 
2-Azido-1-(5-bromothiophen-2-yl)ethanone (A24): ${ }^{1} \mathrm{H}$ NMR (400 MHz, $\left.\mathbf{C D C l}_{3}\right): \delta 7.47$ (d, $J=4.1 \mathrm{~Hz}, 1 \mathrm{H}), 7.14(\mathrm{~d}, J=4.1 \mathrm{~Hz}, 1 \mathrm{H}), 4.38(\mathrm{~s}, 2 \mathrm{H}) ;{ }^{13} \mathbf{C} \mathbf{N M R}\left(\mathbf{1 0 1 M H z}, \mathbf{C D C l}_{3}\right): \delta 185.39$, $142.27,132.87,131.65,124.53,54.67$.

2-Azido-1-(thiazol-2-yl)ethanone(A26): ${ }^{1} \mathrm{H}$ NMR (400 MHz, CDCl3): $\delta 7.41(\mathrm{~d}, J=4.0 \mathrm{~Hz}$, 1H), $7.08(\mathrm{~d}, J=4.0 \mathrm{~Hz}, 1 \mathrm{H}), 2.49$ (s, 2H); ${ }^{13} \mathbf{C}$ NMR (101MHz, CDCl3): $\delta$ 189.66, 132.63, $131.32,122.86,26.30$.

Scheme 3. Synthesis of Saccharin Azide A27

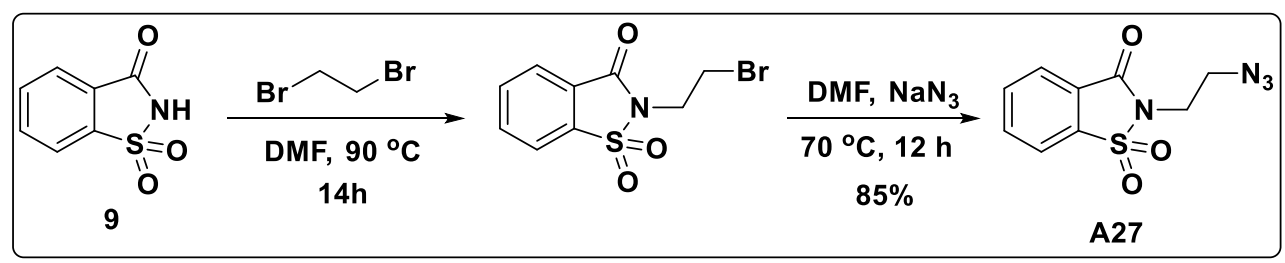

Procedure for Synthesis of Saccharin Azide A27. To a solution of saccharin sodium salt (1.0 $\mathrm{g}, 5.5 \mathrm{mmol})$ in $\mathrm{N}, \mathrm{N}$-Dimethylformamide $(15 \mathrm{~mL})$ was added 1,2-dibromoethane $(1.15 \mathrm{~g}, 6.1$ mmol) and stirred at $90{ }^{\circ} \mathrm{C}$ for $14 \mathrm{~h}$. After complete conversion of saccharin, the insoluble solids were filtered through celite bed, organic solvent was evaporated under reduced pressure and crude was washed with n-hexane and dried in vacuum. The crude content thus obtained was further dissolved in DMF (5 mL) and was charged with sodium azide (1.12 g, $16.5 \mathrm{mmol})$ dissolved in distilled water slowly. The contents were refluxed on a preheated oil bath (70-80 ${ }^{0} \mathrm{C}$ ) till the halide was completely converted to azide (periodically checked on TLC, $12 \mathrm{~h}$ ). After completion of the reaction, the contents were poured onto crushed ice and the product was extracted into ethylacetate. The organic layer was evaporated in vacuum and short silica gel column purification yielded the required azide $\mathbf{A 2 7}$ as pure solid product.

2-(2-azidoethyl)benzo[d]isothiazol-3(2H)-one 1,1-dioxide (A27): ${ }^{1} \mathrm{H}$ NMR (500 MHz, CDCl3): $\delta 8.07(\mathrm{~d}, J=7.4 \mathrm{~Hz}, 1 \mathrm{H}), 7.94(\mathrm{~d}, J=7.4 \mathrm{~Hz}, 1 \mathrm{H}), 7.87(\mathrm{dt}, J=20.8 \& 7.3 \mathrm{~Hz}, 2 \mathrm{H}), 3.96$ (t, J=6.3 Hz, 2H), $3.70(\mathrm{t}, J=6.3 \mathrm{~Hz}, 2 \mathrm{H}) ;{ }^{13} \mathbf{C}$ NMR (126MHz, CDCl$): \delta 159.01,137.60$, $135.16,134.64,127.13,125.50,121.19,48.89,38.14$. 
General Procedure for the Synthesis of Piperazine-Azides A28-A35. In an oven dried clean round bottomed flask, piperazines $(1.0 \mathrm{mmol})$ were taken in dichloromethane and cooled to 0 ${ }^{\circ} \mathrm{C}$. Triethylamine $\left(\mathrm{Et}_{3} \mathrm{~N}\right)(1.5 \mathrm{mmol})$ was added to the contents while stirring the contents and chloroacetyl chloride $(1.5 \mathrm{mmol})$ dissolved in DCM was added dropwise. After complete addition of chloroacetylchloride, the reaction was stirred at room temperature for the complete conversion of starting materials (as indicated on TLC) typically for 4-6 h. After completion of reaction, the solvent was removed under reduced pressure and the contents were washed with water. The crude residue was extracted into dichloromethane $(3 \times 50 \mathrm{~mL})$ and washed with brine solution, dried over sodium sulphate and solvent was removed invacuum. The crude thus obtained was further dissolved in acetone-ice cold water mixture $(20 \mathrm{~mL})$ and was cooled again to $0{ }^{\circ} \mathrm{C}$. This reaction mixture was charged with sodium iodide, stirred for 5-10 mins and sodium azide (1 mmol) was added portion wise, carefully, and stirred overnight for the complete conversion of the acylchloride to azide. After due completion, as checked on TLC, acetone was evaporated on rotary evaporator and the contents were partitioned between water and ethylacetate $(3 \times 50 \mathrm{~mL})$. The organic layer was washed with brine solution, passed over sodium sulphate bed and solvent was removed under reduced pressure. The crude azides were purified via silica gel column chromatography using $n$-hexane:ethylacetate as column eluent (ethylacetate 0 to $60 \%$ ).

Scheme 4. Synthesis of Piperazine-Azides A28- A35

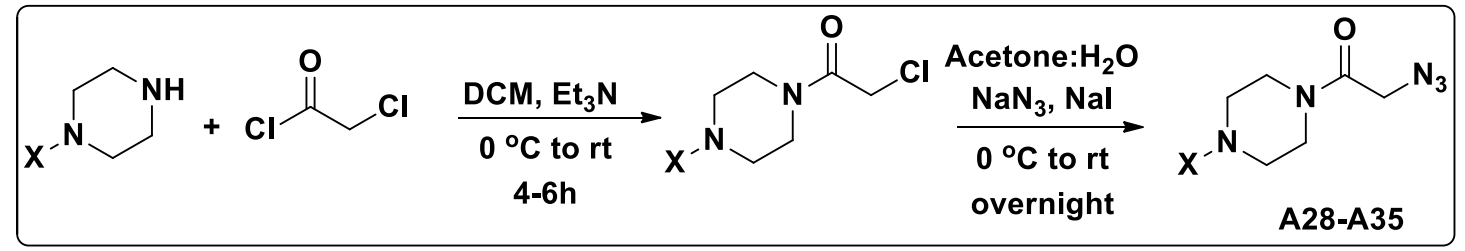

Table S2. Piperazine-Azides - List of Substitutions, Codes and Yields

\begin{tabular}{|c|c|c|c|c|c|c|}
\hline Code & X- & $\begin{array}{c}\text { Overall Yield } \\
(\%)\end{array}$ & Code & X- & $\begin{array}{c}\text { Overall Yield } \\
(\%)\end{array}$ \\
\hline
\end{tabular}




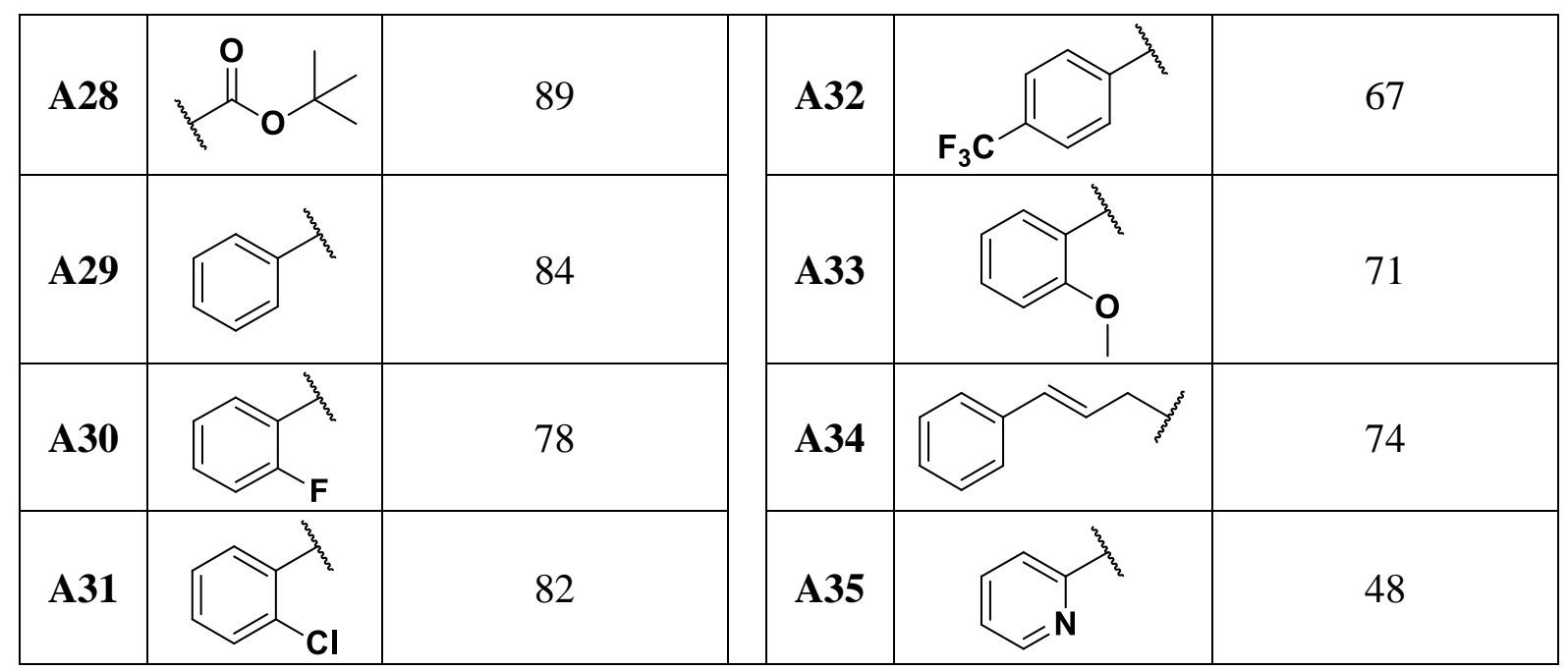

t-Butyl 4-(2-azidoacetyl)piperazine-1-carboxylate (A28): ${ }^{1} \mathrm{H}$ NMR (500 MHz, CDCl3): $\delta$ 3.94 (s, 2H), 3.60 (d, J=5.0 Hz, 2H), 3.46-3.44 (m, 4H), 3.35 (d, J=4.9 Hz, 2H), 1.46 (s, 9H); ${ }^{13}$ C NMR (101MHz, CDCl3): $\delta$ 165.94, 154.55, 80.70, 50.92, 45.12, 28.48.

2-Azido-1-(4-phenylpiperazin-1-yl)ethanone (A29): ${ }^{1} \mathrm{H}$ NMR (400 $\left.\mathrm{MHz}, \mathrm{CDCl}_{3}\right): \delta 7.32$ $7.27(\mathrm{~m}, 2 \mathrm{H}), 6.94-6.91(\mathrm{~m}, 3 \mathrm{H}), 3.98(\mathrm{~s}, 2 \mathrm{H}), 3.80(\mathrm{dd}, J=8.6 \& 3.6 \mathrm{~Hz}, 2 \mathrm{H}), 3.56-3.53(\mathrm{~m}$, 2H), 3.20-3.16 (m, 2H); ${ }^{13}$ C NMR (126MHz, CDCl3): $\delta 165.75,150.85,129.42,120.98$, $116.93,50.85,49.84,49.53,45.23,42.13$.

2-Azido-1-(4-(2-fluorophenyl)piperazin-1-yl)ethanone (A30): ${ }^{1} \mathrm{H}$ NMR (400 MHz, CDCl 3$): \delta$ 7.10-7.02 (m, 2H), 7.01-6.96 (m, 1H), 6.95-6.91 (m, 1H), $3.98(\mathrm{~s}, 2 \mathrm{H}), 3.80(\mathrm{~d}$, $J=16.1 \mathrm{~Hz}, 2 \mathrm{H}), 3.56(\mathrm{~s}, 2 \mathrm{H}), 3.10(\mathrm{t}, J=11.1 \mathrm{~Hz}, 2 \mathrm{H}) ;{ }^{13} \mathbf{C}$ NMR $\left(\mathbf{1 0 1 M H z}, \mathbf{C D C l}_{3}\right): \delta$ 165.77, $154.67,139.39,124.71,123.51,119.40,116.55,116.34,50.85,50.39,45.47,42.30$.

2-Azido-1-(4-(2-chlorophenyl)piperazin-1-yl)ethanone (A31): ${ }^{1} \mathrm{H}$ NMR $(500 \mathrm{MHz}$, CDCl3): $\delta$ 7.40-7.38 (m, 1H), 7.26-7.22 (m, 1H), 7.05-7.00 (m, 2H), 3.99 (s, 2H), 3.84-3.82 (m, 2H), 3.58-3.56 (m, 2H), $3.06(\mathrm{~s}, 4 \mathrm{H}) ;{ }^{13} \mathbf{C}$ NMR (101MHz, CDCl3): $\delta$ 165.86, 148.52, $130.90,129.11,127.88,124.64,120.70,51.57,51.06,50.90,45.61,42.49$.

2-Azido-1-(4-(4-(trifluoromethyl)phenyl)piperazin-1-yl)ethanone (A32): ${ }^{1} \mathrm{H}$ NMR (400 MHz, CDCl $): \delta 7.51(\mathrm{~d}, J=8.5 \mathrm{~Hz}, 2 \mathrm{H}), 6.93$ (d, J=8.6 Hz, 2H), 3.99 (s, 2H), 3.82-3.80 (m, 
2H), 3.58-3.55 (m, 2H), $3.30(\mathrm{~d}, J=4.8 \mathrm{~Hz}, 4 \mathrm{H}) ;{ }^{13} \mathbf{C}$ NMR (101MHz, CDCl 3$): \delta 165.85$, $152.84,126.72,125.99,123.30,121.97,121.65,115.35,50.87,48.47,48.22,44.89,41.78$.

2-Azido-1-(4-(2-methoxyphenyl)piperazin-1-yl)ethanone(A33): ${ }^{1} \mathrm{H}$ NMR (400 MHz, CDCl3): $\delta 7.03(\mathrm{ddd}, J=8.0,7.0 \& 2.1 \mathrm{~Hz}, 1 \mathrm{H}), 6.95-6.85(\mathrm{~m}, 4 \mathrm{H}), 3.97(\mathrm{~s}, 2 \mathrm{H}), 3.86(\mathrm{~s}, 3 \mathrm{H})$, 3.82-3.80 (m, 2H), 3.55-3.53 (m, 2H), $3.05(\mathrm{dd}, J=9.6 \& 4.5 \mathrm{~Hz}, 4 \mathrm{H}) ;{ }^{13} \mathbf{C}$ NMR $(\mathbf{1 0 1 M H z}$ CDCl$\left._{3}\right): \delta 165.65,152.27,140.39,123.80,121.10,118.54,111.41,55.49,50.83,50.68,50.46$, $45.43,42.34$.

(E)-2-Azido-1-(4-cinnamylpiperazin-1-yl)ethanone (A34): ${ }^{1} \mathrm{H}$ NMR (500 $\left.\mathrm{MHz}, \mathrm{CDCl}_{3}\right)$ : $\delta$ $7.38(\mathrm{dd}, J=5.2 \& 3.5 \mathrm{~Hz}, 2 \mathrm{H}), 7.33-7.29(\mathrm{~m}, 2 \mathrm{H}), 7.26-7.22(\mathrm{~m}, 1 \mathrm{H}), 6.53(\mathrm{~d}, J=15.3 \mathrm{~Hz}, 1 \mathrm{H})$, $6.23(\mathrm{dt}, J=15.8 \& 6.8 \mathrm{~Hz}, 1 \mathrm{H}), 3.92(\mathrm{~s}, 2 \mathrm{H}), 3.69-3.67(\mathrm{~m}, 2 \mathrm{H}), 3.41-3.39(\mathrm{~m}, 2 \mathrm{H}), 3.18(\mathrm{dd}$, $\mathrm{J}=6.8 \& 1.3 \mathrm{~Hz}, 2 \mathrm{H}), 2.52-2.50(\mathrm{~m}, 4 \mathrm{H}) ;{ }^{13} \mathbf{C} \mathbf{N M R}(\mathbf{1 2 6 M H z}, \mathbf{C D C l}): \delta 165.44,136.54$ $133.56,128.54,127.64,126.27,125.58,60.69,52.81,52.51,50.49,44.92,41.96$.

2-Azido-1-(4-(pyridin-2-yl)piperazin-1-yl)ethanone (A35): ${ }^{1} \mathrm{H}$ NMR (400 $\left.\mathrm{MHz}, \mathrm{CDCl}_{3}\right)$ : $\delta$ $8.19(\mathrm{~d}, \mathrm{~J}=4.9 \mathrm{~Hz}, 1 \mathrm{H}), 7.54-7.49(\mathrm{~m}, 1 \mathrm{H}), 6.67$ (dd, J=13.0 \& $7.7 \mathrm{~Hz}, 2 \mathrm{H}), 3.98$ (s, 2H), 3.78-

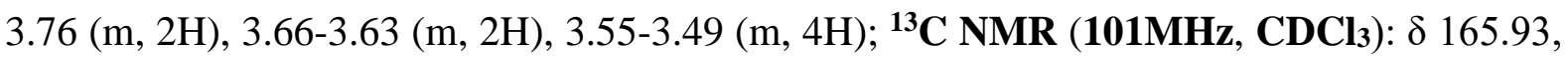
158.92, 148.07, 137.92, 114.28, 107.44, 50.86, 45.33, 45.12, 44.96, 41.76.

General Procedure for Synthesis of Triazoles 10-44. Method A: To a clean, oven dried $50 \mathrm{~mL}$ round bottomed flask, alkyne 8 (1.0 eq), corresponding azide (1.2 eq), $\mathrm{CuSO}_{4} .5 \mathrm{H}_{2} \mathrm{O}(2.0$ eq), sodium-L-ascorbate (2.0 eq) were added to a solution of $t-\mathrm{BuOH}-\mathrm{H}_{2} \mathrm{O}(1: 1, \mathrm{v} / \mathrm{v})$ mixture and stirred overnight at room temperature. The solvent was evaporated on rotary evaporator and the contents were partitioned between water and ethyl acetate. The ethyl acetate layer was collected, passed through sodium sulphate layer and evaporated under reduced pressure. The crude product was then purified using column chromatography to obtain the desired triazoles using $n$-hexane:ethylacetate solvent mixture as column eluent. The corresponding azides and weights taken are mentioned below at the respective compounds data. 
Scheme 5. Synthesis of Usnic Acid Enaminone-coupled Triazoles 10-44

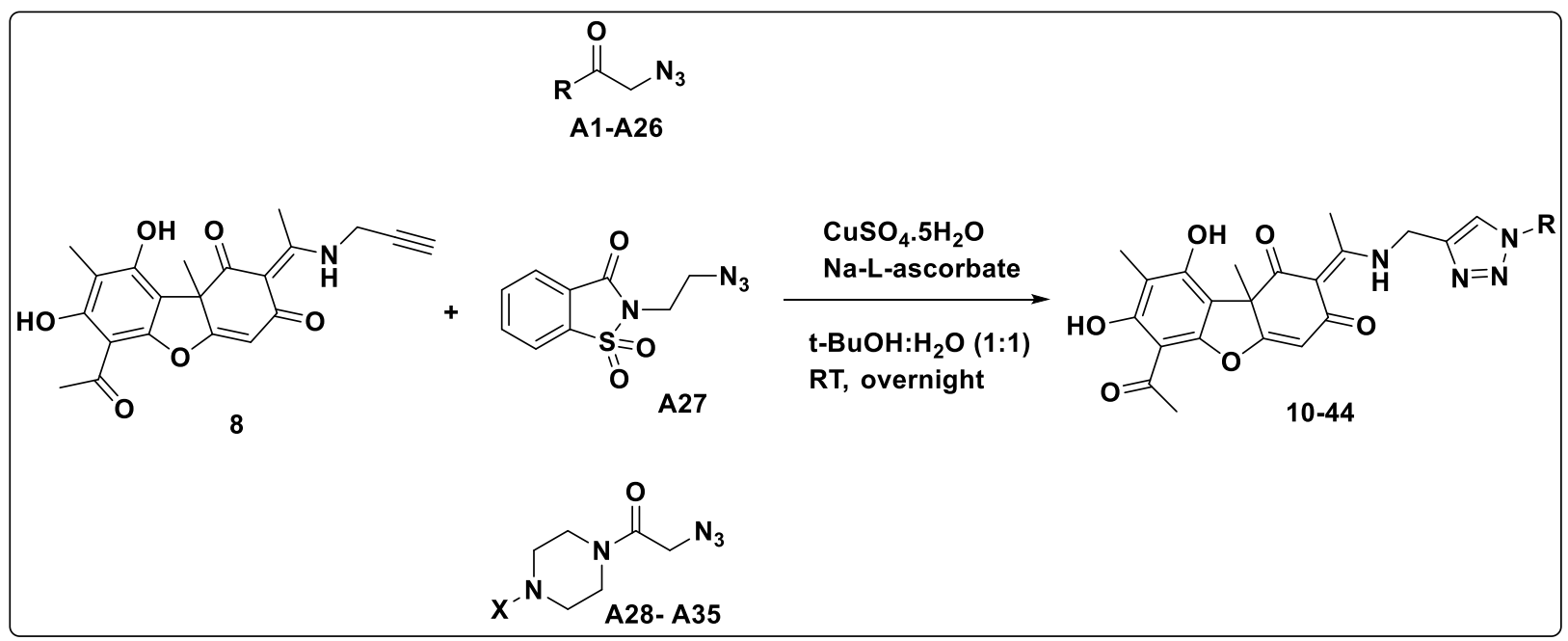

Method B: To a clean, oven dried $50 \mathrm{~mL}$ round bottomed flask, alkyne 8 (1.0 eq), corresponding (thio)mopholine or piperazine azides (1.2 eq), $\mathrm{CuSO}_{4} .5 \mathrm{H}_{2} \mathrm{O}$ (2.0 eq), sodiumL-ascorbate (2.0 eq) were added to a solution of $t-\mathrm{BuOH}-\mathrm{H}_{2} \mathrm{O}(1: 1$ ratio) mixture and stirred for $14-24 \mathrm{~h}$ at room temperature. The solvent was evaporated on rotary evaporator and the contents were partitioned between water and ethyl acetate. The ethyl acetate layer was collected, passed through sodium sulphate layer and evaporated under reduced pressure. The crude product was then purified using column chromatography to obtain the desired triazoles using $n$-hexane:ethylacetate solvent mixture as column eluent (ethylacetate- 0 to $80 \%$ ). The corresponding azides and weights taken are mentioned below at the respective compounds.

(E)-6-Acetyl-7,9-dihydroxy-8,9b-dimethyl-2-(1-(((1-(2-oxo-2-phenylethyl)-1H-1,2,3triazol-4-yl)methyl)amino)ethylidene)dibenzo[b,d]furan-1,3(2H,9bH)-dione $\quad(10)$ : The

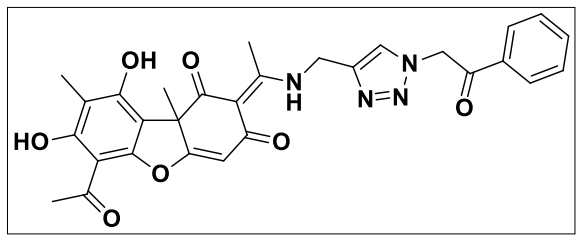
titled compound was synthesized according to the aforementioned general procedure Method A by reacting alkyne 8 (100 mg, $0.26 \mathrm{mmol})$ with azide $\mathbf{A 1}(51 \mathrm{mg}$,

$0.314 \mathrm{mmol})$. The product was obtained as colourless solid. Yield: $61 \mathrm{mg}$ (43\%); M.p.: 114$116{ }^{\circ} \mathrm{C}$; IR spectrum, $\left(v, \mathrm{~cm}^{-1}\right): 3448.04,2928.14,1700.72,1626.82,1556.62,1457.53$, 1370.48, 1318.10, 1285.86, 1229.93, 1189.05, 1139.47, 1064.22; ${ }^{\mathbf{1}} \mathbf{H}$ NMR (400 MHz, 
CDCl3): $\delta 13.86(\mathrm{~s}, 1 \mathrm{H}), 13.35(\mathrm{~s}, 1 \mathrm{H}), 11.87(\mathrm{~s}, 1 \mathrm{H}), 8.00(\mathrm{~d}, J=7.4 \mathrm{~Hz}, 2 \mathrm{H}), 7.80(\mathrm{~s}, 1 \mathrm{H}), 7.69$ (t, J=7.5 Hz, 1H), $7.55(\mathrm{t}, J=7.7 \mathrm{~Hz}, 2 \mathrm{H}), 5.90(\mathrm{~s}, 2 \mathrm{H0}, 5.76(\mathrm{~s}, 1 \mathrm{H}), 4.87$ (s, 2H), $2.76(\mathrm{~s}, 3 \mathrm{H})$, 2.66 (s, 3H), 2.08 (s, 3H), 1.69 (s, 3H); ${ }^{13}$ C NMR (101 MHz, CDCl3): $\delta$ 200.80, 198.64, $190.08,163.62,158.33,155.96,134.97,133.85,129.40,128.75,128.63,128.27,124.15$, 108.19, 105.13, 101.50, 55.71, 39.71, 32.09, 31.42, 18.81, 7.63; ESI-MS: m/z 543.20 [M+H] HRMS $\left(\mathrm{C}_{29} \mathrm{H}_{26} \mathrm{~N}_{4} \mathrm{O}_{7}\right)$ ESI m/z: calcd: $543.1884[\mathrm{M}+\mathrm{H}]^{+}$, found: 543.1874; HPLC Rt: 11.919 $\min$.

(E)-6-Acetyl-7,9-dihydroxy-2-(1-(((1-(2-(4-methoxyphenyl)-2-oxoethyl)-1H-1,2,3-triazol4-yl)methyl)amino)ethylidene)-8,9b-dimethyldibenzo $[b, d]$ furan-1,3(2H,9bH)-dione (11):

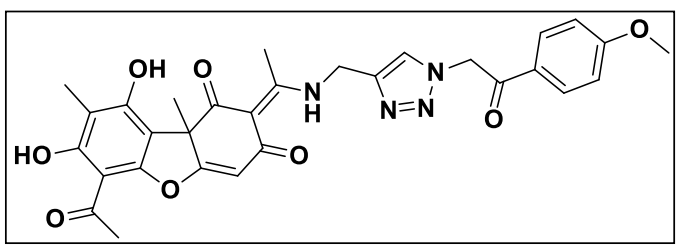

The titled compound was synthesized according to the aforementioned general procedure Method A by reacting alkyne $\mathbf{8}(100 \mathrm{mg}, 0.26 \mathrm{mmol})$ with azide A2 (60 mg, $0.314 \mathrm{mmol})$. The product was obtained as colourless solid. Yield: $110 \mathrm{mg}$ (73\%); M.p.: $116-118{ }^{\circ} \mathrm{C}$; IR spectrum, $\left(v, \mathrm{~cm}^{-1}\right): 3446.59,2928.01,1695.94,1625.01,1555.24$, 1464.41, 1369.58, 1317.81, 1283.67, 1238.48, 1183.22, 1139.38, 1060.97; ${ }^{\mathbf{1}} \mathbf{H}$ NMR (500 MHz, CDCl3): $\delta 13.90$ (s, 1H), 13.35 (s, 1H), 11.90 (s, 1H), 7.97 (d, J=8.8 Hz, 2H), 7.78 (s, 1H), 6.99 (d, J=8.8 Hz, 2H), $5.83(\mathrm{~s}, 2 \mathrm{H}), 5.77(\mathrm{~s}, 1 \mathrm{H}), 4.86(\mathrm{~s}, 2 \mathrm{H}), 3.90(\mathrm{~s}, 3 \mathrm{H}), 2.77$ (s, 3H), 2.66 (s, 3H), 2.09 (s, 3H), $1.70(\mathrm{~s}, 3 \mathrm{H}) ;{ }^{13} \mathbf{C}$ NMR (126 MHz, CDCl 3$): \delta$ 200.81, 198.66, $188.39,175.57,164.94,163.63,158.36,155.98,142.51,130.70,126.81,124.13,114.60$, $108.19,105.15,102.66,102.54,102.39,101.51,55.81,55.34,39.74,32.07,31.41,18.80,7.63$; ESI-MS: m/z $573.35[\mathrm{M}+\mathrm{H}]^{+}$; HRMS $\left(\mathrm{C}_{30} \mathrm{H}_{28} \mathrm{~N}_{4} \mathrm{O}_{8}\right)$ ESI m/z: calcd: $573.1952[\mathrm{M}+\mathrm{H}]^{+}$, found: 573.1978; HPLC Rt: 13.558 min. 
(E)-6-Acetyl-7,9-dihydroxy-2-(1-(((1-(2-(3-methoxyphenyl)-2-oxoethyl)-1H-1,2,3-triazol4-yl)methyl)amino)ethylidene)-8,9b-dimethyldibenzo[b,d]furan-1,3(2H,9bH)-dione (12):

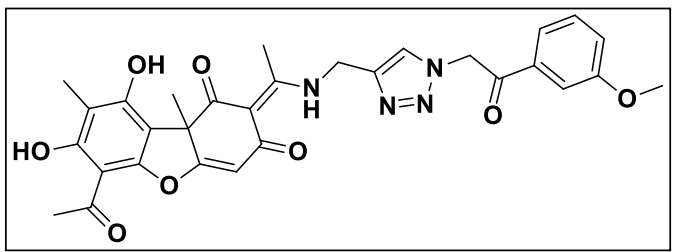

The titled compound was synthesized according to the aforementioned general procedure Method A by reacting alkyne $\mathbf{8}(100 \mathrm{mg}, 0.26 \mathrm{mmol})$ with azide

A3 (60.4 mg, $0.314 \mathrm{mmol})$. The product was obtained as colourless solid. Yield: $93 \mathrm{mg}(62 \%)$; M.p.: $124-126{ }^{\circ} \mathrm{C}$; IR spectrum, $\left(v, \mathrm{~cm}^{-1}\right): 3445.41,2928.57,2362.25,1700.92,1625.82$, 1555.84, 1464.12, 1369.26, 1285.86, 1189.45, 1139.17, 1058.68; ${ }^{1} \mathbf{H}$ NMR (500 MHz, CDCl $): \delta 13.81(\mathrm{~s}, 1 \mathrm{H}), 13.28(\mathrm{~s}, 1 \mathrm{H}), 11.80(\mathrm{~s}, 1 \mathrm{H}), 7.72(\mathrm{~s}, 1 \mathrm{H}), 7.49(\mathrm{~d}, J=7.6 \mathrm{~Hz}, 1 \mathrm{H}), 7.39$ (dd, J=17.2 \& $9.1 \mathrm{~Hz}, 2 \mathrm{H}), 7.14(\mathrm{~d}, J=8.2 \mathrm{~Hz}, 1 \mathrm{H}), 5.81(\mathrm{~s}, 2 \mathrm{H}), 5.70(\mathrm{~s}, 1 \mathrm{H}), 4.80(\mathrm{~s}, 2 \mathrm{H}), 3.80$ (s, 3H), 2.70 (s, 3H), 2.59 (s, 3H), 2.01 (s, 3H), 1.63 (s, 3H); ${ }^{13}$ C NMR (101 MHz, CDCl3): $\delta$ $200.80,198.66,189.96,175.55,163.63,160.36,158.34,155.97,142.61,135.12,130.40$, $124.08,121.28,120.62,112.70,108.20,105.13,101.51,55.79,55.72,39.72,32.09,31.41$, 29.83, 18.80, 7.63; ESI-MS: m/z 573.20 [M+H] $]^{+}$; HRMS $\left(\mathrm{C}_{30} \mathrm{H}_{28} \mathrm{~N}_{4} \mathrm{O}_{8}\right)$ ESI m/z: calcd: 573.1968 [M+H] $]^{+}$, found: 573.1981; HPLC Rt: 14.195 min.

(E)-6-Acetyl-2-(1-(((1-(2-(4-chlorophenyl)-2-oxoethyl)-1H-1,2,3-triazol-4yl)methyl)amino)ethylidene)-7,9-dihydroxy-8,9b-dimethyldibenzo[b,d]furan1,3(2H,9bH)-dione (13): The titled compound was synthesized according to the

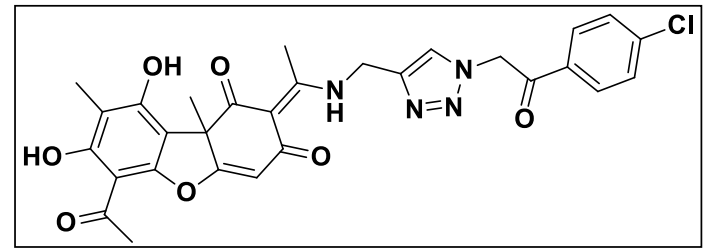
aforementioned general procedure Method A by reacting alkyne $\mathbf{8}(100 \mathrm{mg}, 0.26 \mathrm{mmol})$ with azide A4 (61.5 $\mathrm{mg}, 0.314 \mathrm{mmol})$. The product was obtained as colourless solid. Yield: $97 \mathrm{mg}(64 \%)$; M.p.: $119-121{ }^{\circ} \mathrm{C}$; IR spectrum, $\left(v, \mathrm{~cm}^{-1}\right)$ : $3442.87,2927.63,1701.34,1624.61,1555.95,1464.20,1369.73,1283.98,1230.52,1188.76$, 1138.50, 1061.19; ${ }^{1} \mathbf{H}$ NMR (400 MHz, CDCl $): \delta 13.90$ (s, 1H), 13.35 (s, 1H), 11.86 (s, 1H), $7.95(\mathrm{~d}, J=8.6 \mathrm{~Hz}, 2 \mathrm{H}), 7.78$ (s, 1H), 7.54 (d, J=8.6 Hz, 2H), 5.86 (s, 2H), 5.77 (s, 1H), 4.92- 
$4.81(\mathrm{~m}, 2 \mathrm{H}), 2.77(\mathrm{~s}, 3 \mathrm{H}), 2.66(\mathrm{~s}, 3 \mathrm{H}), 2.09(\mathrm{~s}, 3 \mathrm{H}), 1.70(\mathrm{~s}, 3 \mathrm{H}) ;{ }^{13} \mathrm{C}$ NMR (101 MHz, CDCl$\left._{3}\right): \delta 200.80,198.68,189.01,163.65,158.33,156.03,155.96,142.71,141.72,132.14$, $129.82,129.66,124.05,108.22,105.11,101.52,55.59,39.70,32.09,31.42,18.81,7.64$; ESIMS: m/z 577.20 [M+H] $]^{+}$; HRMS $\left(\mathrm{C}_{29} \mathrm{H}_{25} \mathrm{ClN}_{4} \mathrm{O}_{7}\right)$ ESI m/z: calcd: $577.1469[\mathrm{M}+\mathrm{H}]^{+}$, found: 577.1485; HPLC Rt: 15.526 min.

(E)-6-Acetyl-2-(1-(((1-(2-(3,4-dichlorophenyl)-2-oxoethyl)-1H-1,2,3-triazol-4yl)methyl)amino)ethylidene)-7,9-dihydroxy-8,9b-dimethyldibenzo[b,d]furan1,3(2H,9bH)-dione (14): The titled compound was synthesized according to the

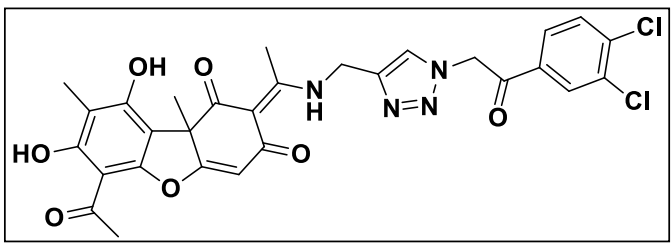
aforementioned general procedure Method A by reacting alkyne $\mathbf{8}(100 \mathrm{mg}, 0.26 \mathrm{mmol})$ with azide A5 (72 mg, $0.314 \mathrm{mmol})$. The product was obtained as colourless solid. Yield: $106 \mathrm{mg}(66 \%)$; M.p.: $128-130{ }^{\circ} \mathrm{C}$; IR spectrum, $\left(v, \mathrm{~cm}^{-1}\right)$ : 3447.33, 2926.92, 1702.93, 1625.66, 1556.35, 1465.08, 1371.56, 1318.32, 1285.14, 1219.17, 1189.02, 1139.25, 1061.11; ${ }^{1} \mathbf{H}$ NMR (400 MHz, CDCl $)$ ) $\delta 13.92$ (s, 1H), 13.35 (s, 1H), 11.87 (s, 1H), $8.08(\mathrm{~d}, J=2.0 \mathrm{~Hz}, 1 \mathrm{H}), 7.83(\mathrm{dd}, J=8.4 \& 2.1 \mathrm{~Hz}, 1 \mathrm{H}), 7.77(\mathrm{~s}, 1 \mathrm{H}), 7.65(\mathrm{~d}, J=8.4 \mathrm{~Hz}, 1 \mathrm{H})$, $5.85(\mathrm{~s}, 2 \mathrm{H}), 5.78(\mathrm{~s}, 1 \mathrm{H}), 4.87(\mathrm{~d}, J=2.6 \mathrm{~Hz}, 2 \mathrm{H}), 2.77(\mathrm{~s}, 3 \mathrm{H}), 2.67(\mathrm{~s}, 3 \mathrm{H}), 2.09(\mathrm{~s}, 3 \mathrm{H}), 1.71$ (s, 3H); ${ }^{13}$ C NMR (101 MHz, CDCl $): \delta 200.79,198.66,188.27,163.63,158.28,155.94$, $142.78,139.88,134.36,133.25,131.54,130.22,127.14,124.03,108.20,105.08,101.50,55.57$, 39.68, 32.08, 31.42, 18.81, 7.64.; ESI-MS: m/z 611.25 [M+H] $]^{+}$HRMS $\left(\mathrm{C}_{29} \mathrm{H}_{24} \mathrm{Cl}_{2} \mathrm{~N}_{4} \mathrm{O}_{7}\right)$ ESI m/z: calcd: $611.1133[\mathrm{M}+\mathrm{H}]^{+}$, found: 611.1095; HPLC Rt: 19.405 min. 
(E)-6-Acetyl-2-(1-(((1-(2-(4-fluorophenyl)-2-oxoethyl)-1H-1,2,3-triazol-4-yl)methyl) amino)ethylidene)-7,9-dihydroxy-8,9b-dimethyldibenzo[b,d]furan-1,3(2H,9bH)-dione

(15): The titled compound was synthesized according to the aforementioned general procedure

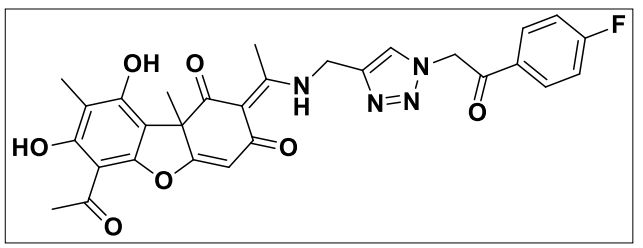

Method A by reacting alkyne $\mathbf{8}(100 \mathrm{mg}, 0.26 \mathrm{mmol})$ with azide $\mathbf{A 6}$ (56.3 $\mathrm{mg}, 0.314 \mathrm{mmol}$ ). The product was obtained as colourless solid. Yield: $90 \mathrm{mg}(61 \%)$; M.p.:

101-103 ${ }^{\circ} \mathrm{C}$; IR spectrum, $\left(v, \mathrm{~cm}^{-1}\right): 3150.68,2928.58,2871.23,1701.90,1625.53,1555.30$, 1464.35, 1419.33, 1369.55, 1284.12, 1231.34, 1188.48, 1138.25, 1060.15; ${ }^{\mathbf{1}} \mathbf{H}$ NMR (500 MHz, CDCl $): \delta 13.83(\mathrm{~s}, 1 \mathrm{H}), 13.33(\mathrm{~s}, 1 \mathrm{H}), 11.82(\mathrm{~s}, 1 \mathrm{H}), 8.02(\mathrm{dd}, J=8.7 \& 5.2 \mathrm{~Hz}, 2 \mathrm{H})$, $7.79(\mathrm{~s}, 1 \mathrm{H}), 7.20(\mathrm{t}, J=8.5 \mathrm{~Hz}, 2 \mathrm{H}), 5.86(\mathrm{~s}, 2 \mathrm{H}), 5.73(\mathrm{~s}, 1 \mathrm{H}), 4.89-4.81(\mathrm{~m}, 2 \mathrm{H}), 2.74(\mathrm{~s}, 3 \mathrm{H})$, 2.64 (s, 3H), 2.06 (s, 3H), 1.67 (s, 3H); ${ }^{13}$ C NMR (126 MHz, CDCl3): $\delta$ 200.79, 198.62, $188.61,175.52,166.75\left(\mathrm{~d}, J_{\mathrm{C}-\mathrm{F}}=258.21\right), 163.59,158.28,155.93,142.61,131.08\left(\mathrm{~d}, J_{\mathrm{C}-\mathrm{F}}=9.6\right.$ $\mathrm{Hz}), 130.27\left(\mathrm{~d}, J_{\mathrm{C}-\mathrm{F}}=2.4 \mathrm{~Hz}\right), 124.14,116.70\left(\mathrm{~d}, J_{\mathrm{C}-\mathrm{F}}=22.2 \mathrm{~Hz}\right), 108.13,105.08,101.46,60.51$, 55.54, 39.66, 32.06, 31.38, 18.78, 7.61; ESI-MS: m/z 561.25 [M+H] $]^{+}$HRMS $\left(\mathrm{C}_{29} \mathrm{H}_{25} \mathrm{FN}_{4} \mathrm{O}_{7}\right)$ ESI m/z: calcd: $561.1811[\mathrm{M}+\mathrm{H}]^{+}$, found: 561.1780; HPLC Rt: $13.586 \mathrm{~min}$.

(E)-6-Acetyl-2-(1-(((1-(2-(3,4-difluorophenyl)-2-oxoethyl)-1H-1,2,3-triazol-4-yl)methyl) amino)ethylidene)-7,9-dihydroxy-8,9b-dimethyldibenzo[b,d]furan-1,3(2H,9bH)-dione (16): The titled compound was synthesized according to the aforementioned general procedure

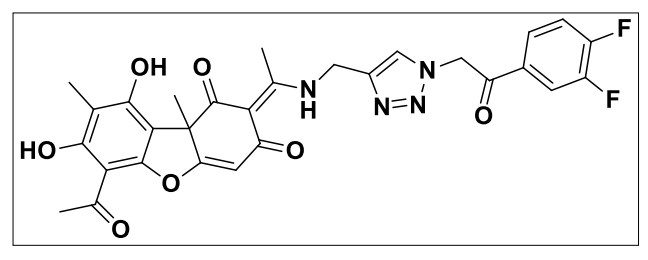
Method A by reacting alkyne 8 (100 mg, $0.26 \mathrm{mmol})$ with azide $\mathbf{A} 7$ (62 $\mathrm{mg}, 0.314 \mathrm{mmol})$. The product was obtained as colourless solid. Yield: $71 \mathrm{mg}(47 \%)$; M.p.: 112-115 ${ }^{\circ} \mathrm{C}$; IR spectrum, $\left(v, \mathrm{~cm}^{-1}\right): 3139.73,2931.65,2871.23,1702.99,1621.48$, 1557.49, 1464.27, $1370.58,1283.84,1188.02,1131.15,1060.55 ;{ }^{1} \mathbf{H}$ NMR (400 MHz, CDCl 3$): \delta 13.88(\mathrm{~s}, 1 \mathrm{H}), 13.34(\mathrm{~s}, 1 \mathrm{H}), 11.83(\mathrm{~s}, 1 \mathrm{H}), 7.87-7.82(\mathrm{~m}, 2 \mathrm{H}), 7.78(\mathrm{~s}, 1 \mathrm{H}), 7.35$ $(\mathrm{dd}, J=16.8 \& 8.6 \mathrm{~Hz}, 1 \mathrm{H}), 5.86(\mathrm{~s}, 2 \mathrm{H}), 5.76(\mathrm{~s}, 1 \mathrm{H}), 4.91-4.81(\mathrm{~m}, 2 \mathrm{H}), 2.76(\mathrm{~s}, 3 \mathrm{H}), 2.65(\mathrm{~s}$, 
3H), 2.08 (s, 3H), 1.69 (s, 3H); ${ }^{13}$ C NMR (101 MHz, CDCl3): $\delta$ 200.80, 198.67, 187.91, 175.56, 163.63, $157.12\left(\mathrm{~d}, J_{\mathrm{C}-\mathrm{F}}=236.1 \mathrm{~Hz}\right), 153.49\left(\mathrm{~d}, J_{\mathrm{C}-\mathrm{F}}=12.3 \mathrm{~Hz}\right), 152.20\left(\mathrm{~d}, J_{\mathrm{C}-\mathrm{F}}=13.2 \mathrm{~Hz}\right)$, $142.74,130.88,125.49\left(\mathrm{dd}, J_{\mathrm{C}-\mathrm{F}}=7.3 \& 3.2 \mathrm{~Hz}\right), 124.06,118.51\left(\mathrm{~d}, J_{\mathrm{C}-\mathrm{F}}=18.1 \mathrm{~Hz}\right), 117.82(\mathrm{~d}$, $\left.J_{\mathrm{C}-\mathrm{F}}=18.3 \mathrm{~Hz}\right), 108.19,105.09,101.50,55.53,39.66,32.08,31.39,18.78,7.62 ;$ ESI-MS: $\mathrm{m} / \mathrm{z}$ $579.20[\mathrm{M}+\mathrm{H}]^{+} ; \mathrm{HRMS}\left(\mathrm{C}_{29} \mathrm{H}_{24} \mathrm{~F}_{2} \mathrm{~N}_{4} \mathrm{O}_{7}\right) \mathrm{ESI} \mathrm{m} / \mathrm{z}$ : calcd: $579.1693[\mathrm{M}+\mathrm{H}]^{+}$, found: 579.1686 ; HPLC Rt: 14.527 min.

\section{(E)-6-Acetyl-7,9-dihydroxy-8,9b-dimethyl-2-(1-(((1-(2-oxo-2-(perfluorophenyl)ethyl)-}

\section{H-1,2,3-triazol-4-yl)methyl)amino)ethylidene)dibenzo[b,d]furan-1,3(2H,9bH)-dione}

(17): The titled compound was synthesized according to the aforementioned general procedure

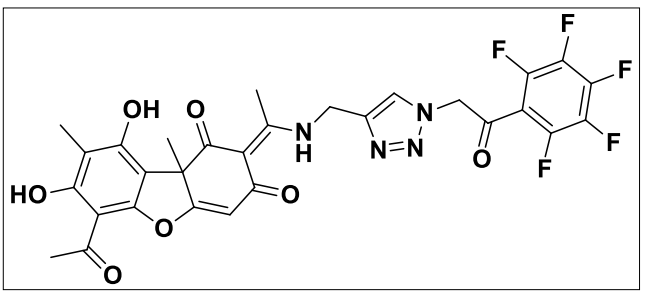

Method A by reacting alkyne 8 (100 mg, $0.26 \mathrm{mmol})$ with azide $\mathbf{A 8}$ (79 $\mathrm{mg}, 0.314 \mathrm{mmol})$. The product was obtained as colourless solid. Yield: $124.5 \mathrm{mg}(75 \%)$;

M.p.: 116-118 ${ }^{\circ} \mathrm{C}$; IR spectrum, $\left(v, \mathrm{~cm}^{-1}\right)$ : 3421.17, 3145.21, 2926.88, 2860.27, 1706.69, 1624.72, 1556.56, 1487.59, 1368.83, 1284.99, 1188.60, 1138.10, 1066.53; ${ }^{\mathbf{1}} \mathbf{H}$ NMR (400 MHz, CDCl3): $\delta 14.04$ (s, 1H), 13.34 (s, 1H), $11.78(\mathrm{~s}, 1 \mathrm{H}), 7.97(\mathrm{~s}, 1 \mathrm{H}), 5.79(\mathrm{~s}, 1 \mathrm{H}), 4.94(\mathrm{~d}$, $J=2.9 \mathrm{~Hz}, 2 \mathrm{H}), 2.80(\mathrm{~s}, 2 \mathrm{H}), 2.70-2.67(2 \mathrm{~s}, 6 \mathrm{H}), 2.09(\mathrm{~s}, 3 \mathrm{H}), 1.71(\mathrm{~s}, 3 \mathrm{H}) ;{ }^{\mathbf{1 3}} \mathrm{C}$ NMR (101 $\left.\mathbf{M H z}, \mathbf{C D C l}_{3}\right): \delta 200.81,191.07\left(\mathrm{~d}, J_{\mathrm{C}-\mathrm{F}}=6.9 \mathrm{~Hz}\right), 198.81,190.69,163.68,157.10\left(\mathrm{~d}, J_{\mathrm{C}-}\right.$ $\mathrm{F}=235.5 \mathrm{~Hz}), 143.11,124.36,108.30,105.02,101.52,39.23,32.60,32.06,31.42,18.79,7.63$; ESI-MS: m/z $615.15[\mathrm{M}+2 \mathrm{H}-\mathrm{F}]^{+} ;$HRMS $\left(\mathrm{C}_{29} \mathrm{H}_{21} \mathrm{~F}_{5} \mathrm{~N}_{4} \mathrm{O}_{7}\right)$ ESI m/z: calcd: 615.1512 $\left[\mathrm{C}_{29} \mathrm{H}_{23} \mathrm{~F}_{4} \mathrm{~N}_{4} \mathrm{O}_{7}, \mathrm{M}+2 \mathrm{H}-\mathrm{F}\right]^{+}$, found: 615.1497; HPLC Rt: 18.267 min. 
(E)-6-Acetyl-7,9-dihydroxy-8,9b-dimethyl-2-(1-(((1-(2-oxo-2-(3-(trifluoromethyl)phenyl) ethyl)-1H-1,2,3-triazol-4-yl)methyl)amino)ethylidene)dibenzo[b,d]furan-1,3(2H,9bH)dione (18): The titled compound was synthesized according to the aforementioned general

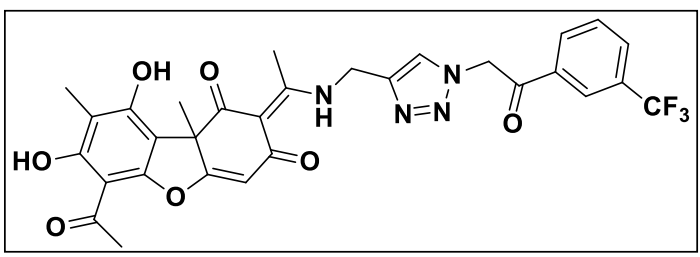
procedure Method A by reacting alkyne 8 (100 $\mathrm{mg}, 0.26 \mathrm{mmol})$ with azide $\mathbf{A 9}(72 \mathrm{mg}, 0.314$ mmol). The product was obtained as colourless

solid. Yield: $67 \mathrm{mg}$ (42\%); M.p.: 96-98 ${ }^{\circ} \mathrm{C}$; IR spectrum, $\left(v, \mathrm{~cm}^{-1}\right)$ : 3413.70, 3145.21, 3084.93, $2925.56,2856.20,1703.64,1625.75,1556.39,1462.37,1370.72,1328.06,1284.36,1176.76$,

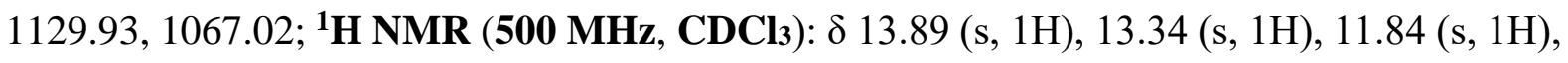
8.25 (s, 1H), 8.19 (d, J=7.8 Hz, 1H), 7.95 (d, J=7.8 Hz, 1H), 7.80 (s, 1H), 7.73 (t, J=7.8 Hz, 1H), $5.93(\mathrm{~s}, 2 \mathrm{H}), 5.77(\mathrm{~s}, 1 \mathrm{H}), 4.87(\mathrm{~s}, 2 \mathrm{H}), 2.77(\mathrm{~s}, 3 \mathrm{H}), 2.66(\mathrm{~s}, 3 \mathrm{H}), 2.08(\mathrm{~s}, 3 \mathrm{H}), 1.70$ (s, 3H); ${ }^{13}$ C NMR (101 MHz, CDCl3): $\delta$ 200.82, 198.70, 189.11, 175.62, 163.65, $157.12\left(\mathrm{~d}, J_{\mathrm{C}-}\right.$ F=236.2 Hz), 134.38, $130.79\left(\mathrm{~d}, J_{\mathrm{C}-\mathrm{F}}=118.7 \mathrm{~Hz}\right), 124.67\left(\mathrm{~d}, J_{\mathrm{C}-\mathrm{F}}=104.1 \mathrm{~Hz}\right), 108.25,105.08$, 101.51, 98.47, 55.75, 39.68, 32.07, 31.40, 29.83, 22.83, 18.82, 14.32, 14.26, 7.63; ESI-MS: m/z $611.25[\mathrm{M}+\mathrm{H}]^{+}$; HRMS $\left(\mathrm{C}_{30} \mathrm{H}_{25} \mathrm{~F}_{3} \mathrm{~N}_{4} \mathrm{O}_{7}\right)$ ESI m/z: calcd: $611.1794[\mathrm{M}+\mathrm{H}]^{+}$, found: 611.1748; HPLC Rt: 16.233 min.

(E)-6-Acetyl-7,9-dihydroxy-8,9b-dimethyl-2-(1-(((1-(2-oxo-2-(4-(trifluoromethyl)phenyl) ethyl)-1H-1,2,3-triazol-4-yl)methyl)amino)ethylidene)dibenzo[b,d]furan-1,3(2H,9bH)dione (19): The titled compound was synthesized according to the aforementioned general

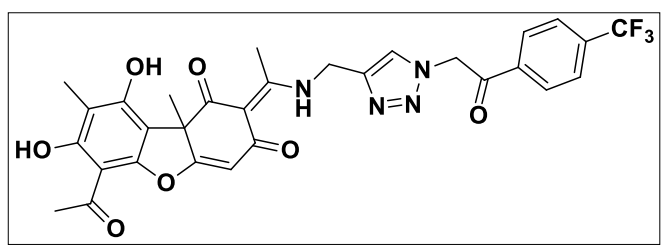

procedure Method A by reacting alkyne 8 (100 mg, $0.26 \mathrm{mmol}$ ) with azide A10 (72 $\mathrm{mg}, 0.314 \mathrm{mmol})$. The product was obtained as colourless solid. Yield: 60 mg (38\%); M.p.: $112-114{ }^{\circ}$ C; IR spectrum, $\left(v, \mathrm{~cm}^{-1}\right)$ : 3445.53, 2969.86, 2929.64, 2860.27, $1703.94,1626.23,1557.07,1464.39,1416.56,1371.68,1324.37,1284.60,1232.41,1179.96$, 1134.36, 1065.20; ${ }^{1} \mathrm{H}$ NMR (400 MHz, CDCl $)$ ) $\delta 13.88$ (s, 1H), 13.35 (s, 1H), 11.83 (s, 1H), 
$8.12(\mathrm{~d}, J=8.2 \mathrm{~Hz}, 2 \mathrm{H}), 7.83(\mathrm{~d}, J=8.3 \mathrm{~Hz}, 1 \mathrm{H}), 7.80(\mathrm{~s}, 1 \mathrm{H}), 5.93(\mathrm{~s}, 2 \mathrm{H}), 5.77(\mathrm{~s}, 1 \mathrm{H}), 4.88$ (d, J=3.1 Hz, 2H), 2.77 (s, 3H), 2.66 (s, 3H), 2.08 (s, 3H), 1.70 (s, 3H); ${ }^{13}$ C NMR (126 MHz, CDCl3): $\delta 200.81,198.70,189.42,175.59,163.65,157.12\left(\mathrm{~d}, J_{\mathrm{C}-\mathrm{F}}=295.0 \mathrm{~Hz}\right), 142.79,136.44$, $136.18\left(\mathrm{~d}, J_{\mathrm{C}-\mathrm{F}}=32.8 \mathrm{~Hz}\right), 128.72,126.50\left(\mathrm{~d}, J_{\mathrm{C}-\mathrm{F}}=3.2 \mathrm{~Hz}\right), 124.08,108.24,105.08,101.52$, 55.85, 39.68, 32.08, 31.40, 18.81, 7.63; ESI-MS: m/z $611.15[\mathrm{M}+\mathrm{H}]^{+} ; \mathrm{HRMS}\left(\mathrm{C}_{30} \mathrm{H}_{25} \mathrm{~F}_{3} \mathrm{~N}_{4} \mathrm{O}_{7}\right)$ ESI m/z: calcd: 611.1795 [M+H] $]^{+}$, found: 611.1779; HPLC Rt: 14.540 min.

\section{(E)-6-Acetyl-7,9-dihydroxy-8,9b-dimethyl-2-(1-(((1-(2-oxo-2-(4-}

\section{(trifluoromethoxy)phenyl)ethyl)-1H-1,2,3-triazol-4-}

yl)methyl)amino)ethylidene)dibenzo $[b, d]$ furan-1,3(2H,9bH)-dione $\quad(20)$ : $\quad$ The titled

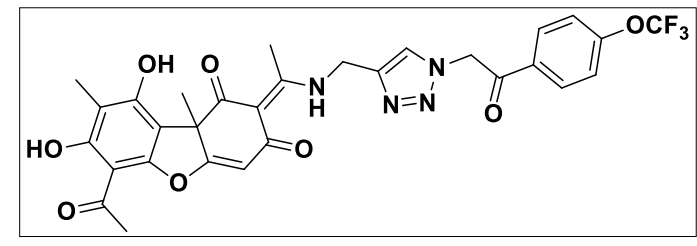

compound was synthesized according to the aforementioned general procedure Method A by reacting alkyne $\mathbf{8}(100 \mathrm{mg}, 0.26 \mathrm{mmol})$ with azide

A11 (77 mg, $0.314 \mathrm{mmol})$. The product was obtained as colourless solid. Yield: $40 \mathrm{mg}(21 \%)$; M.p.: $103-105{ }^{\circ} \mathrm{C}$; IR spectrum, $\left(v, \mathrm{~cm}^{-1}\right): 3448.01,2928.80,1701.74,1624.68,1557.33$, 1464.25, 1371.35, 1263.46, 1219.84, 1062.50; ${ }^{1} \mathbf{H}$ NMR (400 MHz, CDCl3): $\delta 13.86$ (s, 1H), $13.34(\mathrm{~s}, 1 \mathrm{H}), 11.82(\mathrm{~s}, 1 \mathrm{H}), 8.07$ (d, J=8.4 Hz, 2H), 7.79 (s, 1H), 7.38 (d, J=8.0 Hz, 2H), 5.88 (s, 2H), $5.76(\mathrm{~s}, 1 \mathrm{H}), 4.87(\mathrm{~s}, 2 \mathrm{H}), 2.76(\mathrm{~s}, 3 \mathrm{H}), 2.66(\mathrm{~s}, 3 \mathrm{H}), 2.08(\mathrm{~s}, 3 \mathrm{H}), 1.69(\mathrm{~s}, 3 \mathrm{H}) ;{ }^{13} \mathrm{C}$ NMR (126 MHz, CDCl3): $\delta$ 200.81, 198.69, 188.72, 175.60, 163.64, 158.31, 154.96 (d, $J_{\mathrm{C}-}$ $\mathrm{F}=251.7 \mathrm{~Hz}), 142.73,131.90,130.49,124.07,120.97,108.22,105.10,101.51,55.62,39.68$, 32.08, 31.41, 18.81, 7.63; ESI-MS: m/z 627.20 [M+H] $]^{+}$; HRMS $\left(\mathrm{C}_{30} \mathrm{H}_{25} \mathrm{~F}_{3} \mathrm{~N}_{4} \mathrm{O}_{8}\right)$ ESI m/z: calcd: $627.1738[\mathrm{M}+\mathrm{H}]^{+}$, found: 627.1704 ; HPLC Rt: 15.755 min. 
(E)-4-(2-(4-(((1-(6-Acetyl-7,9-dihydroxy-8,9b-dimethyl-1,3-dioxo-1,9b-

dihydrodibenzo[b,d]furan-2(3H)-ylidene)ethyl)amino)methyl)-1H-1,2,3-triazol-1-

yl)acetyl)benzonitrile (21): The titled compound was synthesized according to the

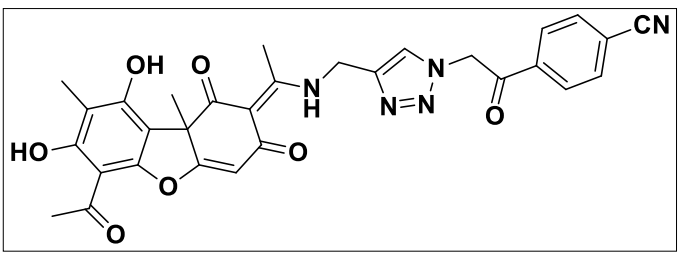
aforementioned general procedure Method A by reacting alkyne $\mathbf{8}(100 \mathrm{mg}, 0.26 \mathrm{mmol})$ with azide A12 (59 $\mathrm{mg}, 0.314 \mathrm{mmol})$. The product was obtained as colourless solid. Yield: $100 \mathrm{mg}(68 \%)$; M.p.: $128-130{ }^{\circ} \mathrm{C}$; IR spectrum, $\left(v, \mathrm{~cm}^{-1}\right)$ : $3422.64,3139.73,2928.81,2860.27,2232.52,1704.54,1624.36,1555.89,1464.11,1370.16$, 1284.45, 1228.15, 1188.97, 1138.41, 1061.42; ${ }^{1} \mathbf{H}$ NMR (500 MHz, CDCl $): \delta 13.89$ (s, 1H), $13.34(\mathrm{~s}, 1 \mathrm{H}), 11.82(\mathrm{~s}, 1 \mathrm{H}), 8.11(\mathrm{~d}, J=8.3 \mathrm{~Hz}, 2 \mathrm{H}), 7.86(\mathrm{~d}, J=8.2 \mathrm{~Hz}, 2 \mathrm{H}), 7.79$ (s, 1H), 5.91 (s, 2H), $5.76(\mathrm{~s}, 1 \mathrm{H}), 4.91-4.83(\mathrm{~m}, 2 \mathrm{H}), 2.76(\mathrm{~s}, 3 \mathrm{H}), 2.66(\mathrm{~s}, 3 \mathrm{H}), 2.08(\mathrm{~s}, 3 \mathrm{H}), 1.69(\mathrm{~s}, 3 \mathrm{H})$; ${ }^{13}$ C NMR (126 MHz, CDCl3): $\delta$ 200.80, 198.67, 189.19, 163.61, 158.26, 155.93, 142.82, $136.65,133.16,128.73,124.04,118.20,117.45,108.19,105.06,101.49,55.81,39.65,32.08$, 31.40, 18.79, 7.63; ESI-MS: m/z 568.20 [M+H] $]^{+}$; HRMS $\left(\mathrm{C}_{30} \mathrm{H}_{25} \mathrm{~N}_{5} \mathrm{O}_{7}\right)$ ESI m/z: calcd: 568.1891 $[\mathrm{M}+\mathrm{H}]^{+}$, found: 568.1830; HPLC Rt: $11.771 \mathrm{~min}$.

(E)-6-Acetyl-7,9-dihydroxy-8,9b-dimethyl-2-(1-(((1-(2-(4-nitrophenyl)-2-oxoethyl)-1H1,2,3-triazol-4-yl)methyl)amino)ethylidene)dibenzo[b,d]furan-1,3(2H,9bH)-dione (22):

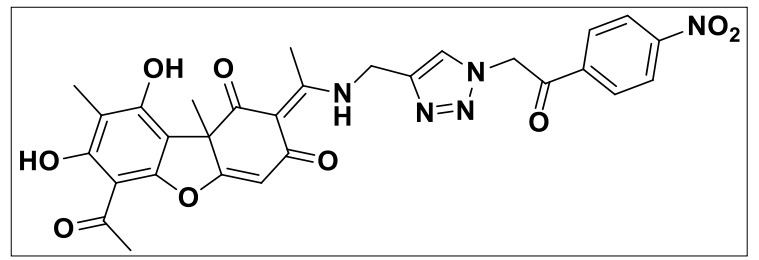

The titled compound was synthesized according to the aforementioned general procedure Method A by reacting alkyne 8 (100 $\mathrm{mg}, 0.26$ mmol) with azide A13 (65 mg, $0.314 \mathrm{mmol}$ ). The product was obtained as colourless solid. Yield: 50 mg (28\%); M.p.: $135-137{ }^{\circ} \mathrm{C}$; IR spectrum, $\left(v, \mathrm{~cm}^{-1}\right): 3447.77,3145.21,2929.43$, $2865.75,1702.79,1624.03,1557.00,1463.71,1355.27,1284.26,1227.92,1190.68,1137.31$,

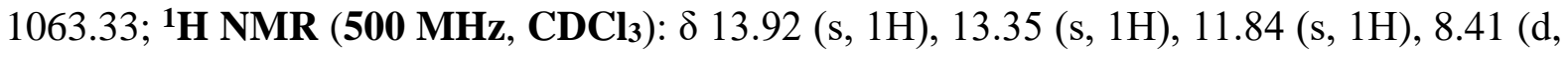
$J=8.7 \mathrm{~Hz}, 2 \mathrm{H}), 8.19$ (d, J=8.7 Hz, 2H), $7.80(\mathrm{~s}, 1 \mathrm{H}), 5.94$ (s, 2H), 5.78 (s, 1H), 4.88 (s,2H), 
$2.77(\mathrm{~s}, 3 \mathrm{H}), 2.67(\mathrm{~s}, 3 \mathrm{H}), 2.09$ (s, 3H), $1.71(\mathrm{~s}, 3 \mathrm{H}) ;{ }^{\mathbf{1 3}} \mathbf{C}$ NMR (126 MHz, CDCl$): \delta 200.82$, $188.98,163.65,158.29,155.95,151.38,138.08,129.49,124.60,123.99,108.26,105.07$, 101.52, 55.95, 39.67, 32.07, 31.43, 18.83, 7.64.; ESI-MS: m/z 588.20 [M+H] $]^{+}$; HRMS $\left(\mathrm{C}_{29} \mathrm{H}_{25} \mathrm{~N}_{5} \mathrm{O}_{9}\right) \mathrm{ESI} \mathrm{m} / \mathrm{z}$ : calcd: 588.1748 [M+H] $]^{+}$, found: 588.1731 ; HPLC Rt: $8.590 \mathrm{~min}$.

(E)-2-(1-(((1-(2-([1,1'-Biphenyl]-4-yl)-2-oxoethyl)-1H-1,2,3-triazol-4-yl)methyl)amino) ethylidene)-6-acetyl-7,9-dihydroxy-8,9b-dimethyldibenzo[b,d]furan-1,3(2H,9bH)-dione (23): The titled compound was synthesized according to the aforementioned general procedure

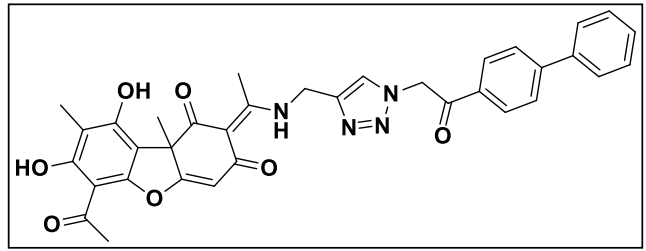
Method A by reacting alkyne 8 (100 $\mathrm{mg}, 0.26 \mathrm{mmol})$ with azide $\mathbf{A 1 4}$ (75 $\mathrm{mg}, 0.314 \mathrm{mmol})$. The product was obtained as colourless solid. Yield: $98 \mathrm{mg}(61 \%)$;

M.p.: 122-124 ${ }^{\circ} \mathrm{C}$; IR spectrum, $\left(v, \mathrm{~cm}^{-1}\right): 3444.43,2926.37,2865.75,1698.80,1625.79$, $1555.44,1464.80,1420.64,1369.09,1317.46,1286.10,1234.49,1188.62,1138.90,1118.04$, 1058.57; ${ }^{1}$ H NMR (400 MHz, CDCl $): \delta 13.93$ (s, 1H), 13.36 (s, 1H), 11.90 (s, 1H), 8.08 (d, $J=8.3 \mathrm{~Hz}, 2 \mathrm{H}), 7.80(\mathrm{~d}, J=5.4 \mathrm{~Hz}, 2 \mathrm{H}), 7.76(\mathrm{~s}, 1 \mathrm{H}), 7.65(\mathrm{~d}, J=6.9 \mathrm{~Hz}, 2 \mathrm{H}), 7.47$ (dt, $J=18.6$ \& $6.9 \mathrm{~Hz}, 3 \mathrm{H}), 5.93(\mathrm{~s}, 2 \mathrm{H}), 5.79(\mathrm{~s}, 1 \mathrm{H}), 4.88(\mathrm{~d}, \mathrm{~J}=4.5 \mathrm{~Hz}, 2 \mathrm{H}), 2.78(\mathrm{~s}, 3 \mathrm{H}), 2.67$ (s, 3H), 2.10 (s, 3H), 1.71 (s, 3H); ${ }^{13}$ C NMR (101 MHz, CDCl3): $\delta$ 200.80, 198.65, 189.64, 175.56, $163.63,158.34,155.97,147.67,142.59,139.30,132.46,129.25,128.89,127.91,127.42$, 124.14, 108.19, 105.13, 101.50, 55.70, 39.73, 32.09, 31.41, 18.82, 7.64.; ESI-MS: m/z 619.20 $[\mathrm{M}+\mathrm{H}]^{+}$; HRMS $\left(\mathrm{C}_{35} \mathrm{H}_{30} \mathrm{~N}_{4} \mathrm{O}_{7}\right)$ ESI m/z: calcd: $619.2218[\mathrm{M}+\mathrm{H}]^{+}$, found: 619.2197; HPLC Rt: $20.514 \mathrm{~min}$. 
(E)-6-Acetyl-2-(1-(((1-(2-(anthracen-9-yl)-2-oxoethyl)-1H-1,2,3-triazol-4-yl)methyl) amino)ethylidene)-7,9-dihydroxy-8,9b-dimethyldibenzo[b,d]furan-1,3(2H,9bH)-dione

(24): The titled compound was synthesized according to the aforementioned general procedure

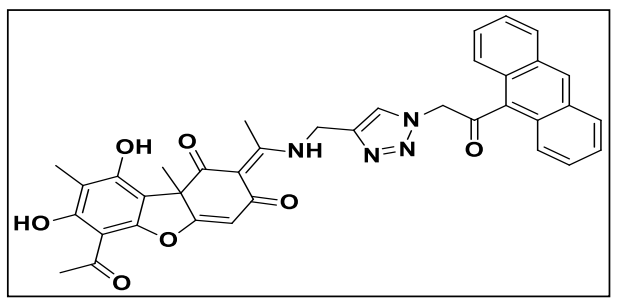

Method A by reacting alkyne $8(100 \mathrm{mg}, 0.26 \mathrm{mmol})$ with azide $\mathbf{A 1 5}$ (82 $\mathrm{mg}, 0.314 \mathrm{mmol}$ ). The product was obtained as colourless solid. Yield: $50 \mathrm{mg}(30 \%)$; M.p.: 146-148 ${ }^{\circ} \mathrm{C}$; IR spectrum, $\left(v, \mathrm{~cm}^{-1}\right): 3450.29,3139.73$, $2927.03,2860.27,1699.81,1624.67,1555.76,1461.40,1369.61,1319.34,1284.71,1189.46$, 1138.30, 1062.54; ${ }^{1} \mathbf{H}$ NMR (500 MHz, CDCl $): \delta 13.93$ (s, 1H), 13.36 (s, 1H), 11.88 (s, 1H), $8.60(\mathrm{~s}, 1 \mathrm{H}), 8.08$ (d, J=8.4 Hz, 2H), $7.94(\mathrm{~s}, 1 \mathrm{H}), 7.87$ (d, J=8.6 Hz, 2H), 7.58 (dt, J=14.9 \& $7.0 \mathrm{~Hz}, 4 \mathrm{H}), 5.78(\mathrm{~s}, 3 \mathrm{H}), 4.89$ (s, 2H), $2.76(\mathrm{~s}, 3 \mathrm{H}), 2.67$ (s, 3H), $2.10(\mathrm{~s}, 3 \mathrm{H}), 1.71(\mathrm{~s}, 3 \mathrm{H})$; ${ }^{13}$ C NMR (126MHz, CDCl3): $\delta$ 200.81, 200.12, 163.65, 158.33, 155.96, 134.26, 131.14, $131.00,130.39,129.33,128.19,127.68,126.07,124.09,123.54,108.23,105.12,101.52,60.57$, 39.67, 31.42, 18.80, 7.64; ESI-MS: m/z 643.25 [M+H] $]^{+}$HRMS $\left(\mathrm{C}_{37} \mathrm{H}_{30} \mathrm{~N}_{4} \mathrm{O}_{7}\right)$ ESI m/z: calcd: 643.2207 [M+H] $]^{+}$, found: 643.2187; HPLC Rt: 23.894 min.

\section{(E)-2-(1-(((1-(2-(9H-Fluoren-2-yl)-2-oxoethyl)-1H-1,2,3-triazol-4-yl)methyl)amino)}

ethylidene)-6-acetyl-7,9-dihydroxy-8,9b-dimethyldibenzo[b,d]furan-1,3(2H,9bH)-dione

(25): The titled compound was synthesized according to the aforementioned general procedure

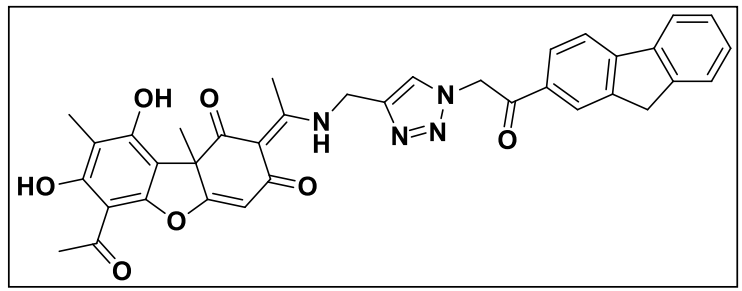

Method A by reacting alkyne 8 (100 mg, 0.26 mmol) with azide A16 (78.4 mg, $0.314 \mathrm{mmol})$. The product was obtained as colourless solid.

Yield: $110 \mathrm{mg}(67 \%)$; M.p.: $136-138{ }^{\circ} \mathrm{C}$; IR spectrum, $\left(v, \mathrm{~cm}^{-1}\right)$ : 3443.89, 3128.77, 2925.96, 2876.71, 1697.01, 1625.03, 1556.18, 1465.71, 1422.15, 1370.34, 1317.15, 1288.09, 1251.76, 1188.87, 1139.70, 1119.00, 1059.43; ${ }^{1} \mathbf{H}$ NMR (500 MHz, CDCl $): \delta 13.88(\mathrm{~s}, 1 \mathrm{H}), 13.34$ (s, 1H), 11.87 (s, 1H), 8.13 (d, J=8.8 Hz, 1H), 8.01 (d, J=7.9 Hz, 1H), 7.88-7.83 (m, 3H), 7.59 (d, 
$J=6.4 \mathrm{~Hz}, 1 \mathrm{H}), 7.45-7.40(\mathrm{~m}, 2 \mathrm{H}), 5.94(\mathrm{~s}, 2 \mathrm{H}), 5.74(\mathrm{~s}, 1 \mathrm{H}), 4.91-4.83(\mathrm{~m}, 2 \mathrm{H}), 3.96(\mathrm{~s}, 2 \mathrm{H})$, 2.77 (s, 3H), 2.63 (s, 3H), 2.08 (s, 3H), 1.68 (s, 3H); ${ }^{13} \mathbf{C}$ NMR (101 MHz, CDCl3): $\delta$ 200.76, $189.76,163.59,158.28,155.92,148.31,144.72,143.97,142.52,140.00,132.02,128.88$, $127.64,127.43,125.49,124.89,124.24,121.32,120.31,108.14,105.09,101.47,55.76,39.74$, 37.03, 32.08, 31.38, 18.80, 7.63; ESI-MS: m/z $631.30[\mathrm{M}+\mathrm{H}]^{+} ; \mathrm{HRMS}\left(\mathrm{C}_{36} \mathrm{H}_{30} \mathrm{~N}_{4} \mathrm{O}_{7}\right) \mathrm{ESI} \mathrm{m} / \mathrm{z}$ : calcd: $631.2291[\mathrm{M}+\mathrm{H}]^{+}$, found: 631.2278; HPLC Rt: $21.881 \mathrm{~min}$.

(E)-6-Acetyl-7,9-dihydroxy-8,9b-dimethyl-2-(1-(((1-(2-(naphthalen-1-yl)-2-oxoethyl)-1H1,2,3-triazol-4-yl)methyl)amino)ethylidene)dibenzo[b,d]furan-1,3(2H,9bH)-dione

(26):

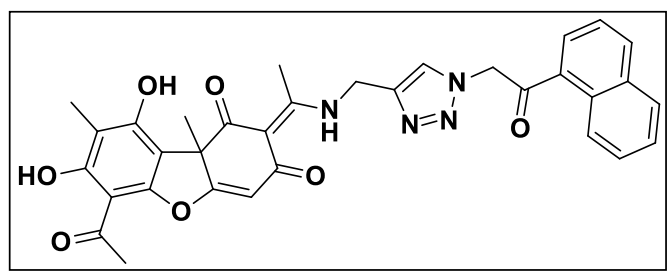

The titled compound was synthesized according to the aforementioned general procedure Method A by reacting alkyne $\mathbf{8}(100 \mathrm{mg}, 0.26 \mathrm{mmol})$ with azide A17 (70 mg, $0.314 \mathrm{mmol})$. The product was obtained as colourless solid. Yield: $73 \mathrm{mg}$ (47\%); M.p.: $124-126{ }^{\circ} \mathrm{C}$; IR spectrum, $\left(v, \mathrm{~cm}^{-1}\right): 3449.82,3145.21,2927.62,2860.27,1697.32$, $1624.80,1555.08,1465.04,1369.45,1284.27,1237.07,1187.91,1139.10,1060.94 ;{ }^{1} \mathbf{H}$ NMR (400 MHz, CDCl3): $\delta 13.94$ (s, 1H), 13.35 (s, 1H), 11.88 (s, 1H), 8.75 (d, J=8.4 Hz, 1H), 8.13 (d, J=8.1 Hz, 1H), 8.07 (d, J=6.8 Hz, 1H), 7.92 (d, J=7.9 Hz, 1H), 7.86 (s, 1H), 7.66-7.57 (m, 3H), $5.95(\mathrm{~s}, 2 \mathrm{H}), 5.78(\mathrm{~s}, 1 \mathrm{H}), 4.89(\mathrm{~s}, 2 \mathrm{H}), 2.78(\mathrm{~s}, 3 \mathrm{H}), 2.67(\mathrm{~s}, 3 \mathrm{H}), 2.10(\mathrm{~s}, 3 \mathrm{H}), 1.71(\mathrm{~s}$, 3H); ${ }^{13}$ C NMR (101 MHz, CDCl $): \delta 200.80,198.65,192.74,163.63,158.33,155.96,135.27$, $134.23,131.08,130.47,129.22,129.11,128.92,127.28,125.55,124.42,108.19,105.13$, 101.51, 57.36, 39.76, 32.08, 31.42, 18.82, 7.64; ESI-MS: m/z 593.30 [M+H] ; HRMS $\left(\mathrm{C}_{33} \mathrm{H}_{28} \mathrm{~N}_{4} \mathrm{O}_{7}\right) \mathrm{ESI} \mathrm{m} / \mathrm{z}$ : calcd: 593.2069 [M+H] $]^{+}$, found: 593.2031; HPLC Rt: $18.274 \mathrm{~min}$. 
(E)-6-Acetyl-7,9-dihydroxy-8,9b-dimethyl-2-(1-(((1-(2-(naphthalen-2-yl)-2-oxoethyl)-1H-

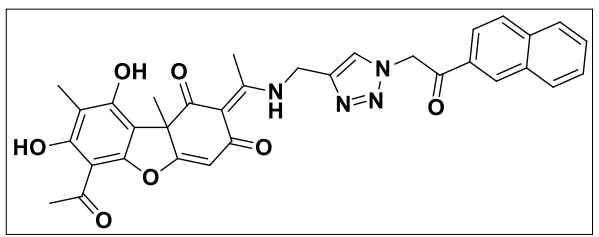

The titled compound was synthesized according to the aforementioned general procedure Method A by reacting alkyne 8 (100 mg, $0.26 \mathrm{mmol}$ ) with azide $\mathbf{A 1 8}$ (66.4 mg,

$0.314 \mathrm{mmol}$ ). The product was obtained as colourless solid. Yield: $35 \mathrm{mg}$ (23\%); M.p.: 122$124{ }^{\circ} \mathrm{C}$; IR spectrum, $\left(v, \mathrm{~cm}^{-1}\right): 3447.09,2829.97,1698.06,1624.05,1556.29,1466.19$, 1369.91, 1283.36, 1188.30, 1133.58, 1060.98; ${ }^{\mathbf{1}} \mathbf{H}$ NMR (400 MHz, CDCl $): \delta 13.91(\mathrm{~s}, 1 \mathrm{H})$, $13.35(\mathrm{~s}, 1 \mathrm{H}), 11.88(\mathrm{~s}, 1 \mathrm{H}), 8.53(\mathrm{~s}, 1 \mathrm{H}), 8.02-8.00(\mathrm{~m}, 2 \mathrm{H}), 7.96(\mathrm{~d}, J=8.7 \mathrm{~Hz}, 1 \mathrm{H}), 7.91(\mathrm{~d}$, $J=8.1 \mathrm{~Hz}, 1 \mathrm{H}), 7.84(\mathrm{~s}, 1 \mathrm{H}), 7.65(\mathrm{dtd}, J=16.2,7.0 \& 1.2 \mathrm{~Hz}, 2 \mathrm{H}), 6.03(\mathrm{~s}, 2 \mathrm{H}), 5.77(\mathrm{~s}, 1 \mathrm{H})$, 4.93-4.83 (m, 2H), $2.78(\mathrm{~s}, 3 \mathrm{H}), 2.66(\mathrm{~s}, 3 \mathrm{H}), 2.09$ (s, 3H), $1.70(\mathrm{~s}, 3 \mathrm{H}) ;{ }^{13} \mathbf{C}$ NMR (126 MHz, CDCl3): $\delta 200.81,190.01,163.63,155.96,136.37,132.47,131.16,130.45,129.87,129.70$, $129.48,128.12,127.62,124.16,123.27,105.14,101.51,55.74,39.75,32.07,31.43,18.83$, 7.64; ESI-MS: m/z 593.25 [M+H] $]^{+}$; HRMS $\left(\mathrm{C}_{33} \mathrm{H}_{28} \mathrm{~N}_{4} \mathrm{O}_{7}\right)$ ESI m/z: calcd: $593.2070[\mathrm{M}+\mathrm{H}]^{+}$, found: 593.2031; HPLC Rt: 17.946 min.

(E)-6-Acetyl-7,9-dihydroxy-2-(1-(((1-(2-(6-methoxynaphthalen-2-yl)-2-oxoethyl)-1H-

\section{1,2,3-triazol-4-yl)methyl)amino)ethylidene)-8,9b-dimethyldibenzo[b,d]furan-1,3}

$(2 \mathrm{H}, 9 b \mathbf{H})$-dione (28): The titled compound was synthesized according to the aforementioned

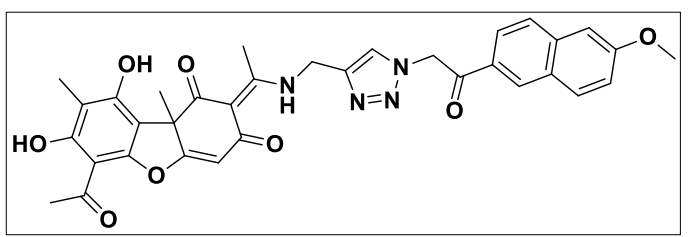

general procedure Method A by reacting alkyne $\mathbf{8}$ (100 mg, $0.26 \mathrm{mmol}$ ) with azide $\mathbf{A 1 9}$ (75.8 mg, $0.314 \mathrm{mmol})$. The product was obtained as colourless solid. Yield: $50 \mathrm{mg}$ (31\%); M.p.: $194-196{ }^{\circ} \mathrm{C}$; IR spectrum, $\left(v, \mathrm{~cm}^{-1}\right)$ : 3448.13, $2930.41,1692.30,1632.25,1543.45,1456.30,1358.94,1335.25,1314.52,1288.99,1220.44$, 1190.88, 1143.60, 1117.92, 1069.70, 1039.78; ${ }^{1} \mathbf{H}$ NMR (400 MHz, CDCl3): $\delta 13.89$ (s, 1H), $13.34(\mathrm{~s}, 1 \mathrm{H}), 11.85(\mathrm{~s}, 1 \mathrm{H}), 8.45(\mathrm{~s}, 1 \mathrm{H}), 8.30(\mathrm{~d}, J=8.3 \mathrm{~Hz}, 1 \mathrm{H}), 8.03$ (d, J=8.2 Hz, 1H), 7.96 
(d, J=8.7 Hz, 1H), $7.86(\mathrm{~s}, 1 \mathrm{H}), 7.36(\mathrm{~d}, J=8.8 \mathrm{~Hz}, 1 \mathrm{H}), 6.00(\mathrm{~s}, 2 \mathrm{H}), 5.76(\mathrm{~s}, 1 \mathrm{H}), 4.89(\mathrm{~s}, 2 \mathrm{H})$, $4.08(\mathrm{~s}, 3 \mathrm{H}), 2.77(\mathrm{~s}, 3 \mathrm{H}), 2.65(\mathrm{~s}, 4 \mathrm{H}), 2.09(\mathrm{~s}, 3 \mathrm{H}), 1.68(\mathrm{~s}, 3 \mathrm{H}) ;{ }^{\mathbf{1 3}} \mathbf{C}$ NMR (101 MHz, $\left.\mathbf{C D C l}_{3}\right): \delta 200.80,198.63,189.49,163.61,158.28,156.69,155.92,136.23,131.18,130.67$, $129.50,128.44,127.61,125.01,114.57,108.73,108.16,105.08,101.48,57.10,55.70,39.75$, 32.09, 31.42, 18.83, 7.65.; ESI-MS: m/z $623.45[\mathrm{M}+\mathrm{H}]^{+}$; HRMS $\left(\mathrm{C}_{34} \mathrm{H}_{30} \mathrm{~N}_{4} \mathrm{O}_{8}\right)$ ESI m/z: calcd: 623.1077 [M+H] $]^{+}$, found 623.1025; HPLC Rt: 10.884 min.

(E)-6-Acetyl-7,9-dihydroxy-8,9b-dimethyl-2-(1-(((1-(2-morpholino-2-oxoethyl)-1H-1,2,3triazol-4-yl)methyl)amino)ethylidene)dibenzo[b,d]furan-1,3(2H,9bH)-dione $\quad(29)$ : The

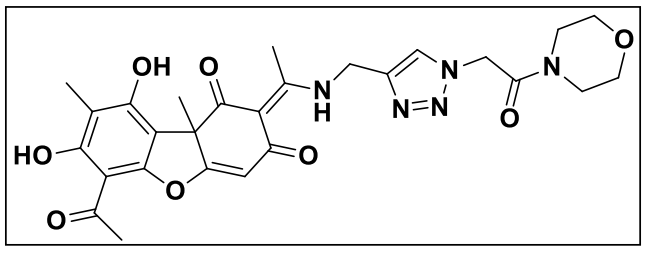
titled compound was synthesized according to the aforementioned general procedure Method B by reacting alkyne $\mathbf{8}(100 \mathrm{mg}, 0.26 \mathrm{mmol})$ with azide $\mathbf{A 2 0}$ (64 mg, $0.314 \mathrm{mmol}$ ). The product was obtained as colourless solid. Yield: $70 \mathrm{mg}$ (48\%); M.p.: $112-114{ }^{\circ} \mathrm{C}$; IR spectrum, $\left(v, \mathrm{~cm}^{-1}\right): 3448.29,2924.70,2861.43,2107.04,1625.20,1555.50$, 1463.56, 1369.92, 1281.58, 1239.44, 1189.39, 1115.07, 1063.84; ${ }^{1} \mathbf{H}$ NMR (400 MHz, CDCl3): $\delta 13.89$ (s, 1H), 13.35 (s, 1H), $11.88(\mathrm{~s}, 1 \mathrm{H}), 7.82(\mathrm{~s}, 1 \mathrm{H}), 5.79(\mathrm{~s}, 1 \mathrm{H}), 5.27(\mathrm{~s}, 2 \mathrm{H})$, $4.83(\mathrm{~s}, 2 \mathrm{H}), 3.73(\mathrm{dd}, J=10.0$ \& $4.8 \mathrm{~Hz}, 4 \mathrm{H}), 3.66(\mathrm{~d}, J=4.7 \mathrm{~Hz}, 2 \mathrm{H}), 3.59$ (d, $J=4.2 \mathrm{~Hz}, 2 \mathrm{H})$, 2.76 (s, 3H), 2.67 (s, 3H), 2.10 (s, 3H), 1.71 (s, 3H); $\left.{ }^{13} \mathbf{C ~ N M R ~ ( 1 2 6 ~ M H z , ~ C D C l} 3\right): \delta 200.80$, $198.65,163.64,163.35,158.33,155.97,108.20,105.12,101.51,66.71,66.44,50.97,45.86$, 42.74, 39.69, 32.08, 31.41, 22.78, 18.78, 7.62; ESI-MS: m/z 552.25 [M+H] $]^{+}$; HRMS $\left(\mathrm{C}_{27} \mathrm{H}_{29} \mathrm{~N}_{5} \mathrm{O}_{8}\right)$ ESI m/z: calcd: 552.2116 [M+H] $]^{+}$, found: 552.2095; HPLC Rt: $8.808 \mathrm{~min}$.

(E)-6-Acetyl-7,9-dihydroxy-8,9b-dimethyl-2-(1-(((1-(2-oxo-2-thiomorpholinoethyl)-1H1,2,3-triazol-4-yl)methyl)amino)ethylidene)dibenzo[b,d]furan-1,3(2H,9bH)-dione (30):

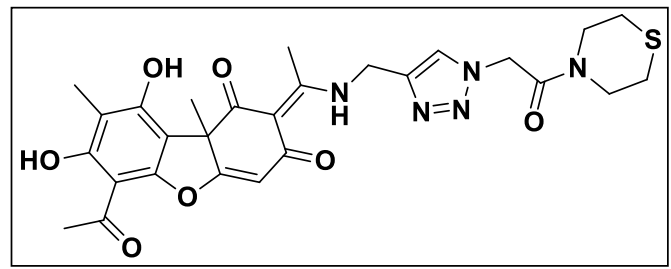

The titled compound was synthesized according to the aforementioned general procedure Method B by reacting alkyne $\mathbf{8}(100 \mathrm{mg}, 0.26 \mathrm{mmol})$ with azide 
A21 (60 mg, $0.314 \mathrm{mmol})$. The product was obtained as colourless solid. Yield: $28.0 \mathrm{mg}$ (19\%); M.p.: $94-96{ }^{\circ} \mathrm{C}$; IR spectrum, $\left(v, \mathrm{~cm}^{-1}\right)$ : 3446.27, 3145.21, 2923.03, 2860.27, 2816.44, 1667.43, $1623.88,1555.33,1461.94,1370.02,1282.09,1233.31,1189.07,1140.37,1060.69 ;{ }^{1} \mathbf{H}$ NMR (500 MHz, CDCl3): $\delta 13.89$ (s, 1H), 13.35 (s, 1H), 11.88 (s, 1H), 7.79 (s, 1H), 5.78 (s, 1H), $5.26(\mathrm{~s}, 2 \mathrm{H}), 4.83(\mathrm{t}, J=5.0 \& 1 \mathrm{~Hz}, 2 \mathrm{H}), 3.92-3.90(\mathrm{~m}, 2 \mathrm{H}), 3.85-3.83(\mathrm{~m}, 2 \mathrm{H}), 2.75(\mathrm{~s}, 3 \mathrm{H})$, $2.67(\mathrm{~s}, 7 \mathrm{H}), 2.09(\mathrm{~s}, 3 \mathrm{H}), 1.71(\mathrm{~s}, 3 \mathrm{H}) ;{ }^{13} \mathrm{C}$ NMR (101 MHz, CDCl$): \delta$ 200.82, 163.64, $163.21,158.34,155.98,124.02,108.20,105.13,101.51,51.20,48.26,45.22,39.66,36.80$, 32.08, 31.42, 27.96, 27.35, 18.78, 7.63; ESI-MS: m/z 568.45 $[\mathrm{M}+\mathrm{H}]^{+} ;$HRMS $\left(\mathrm{C}_{27} \mathrm{H}_{29} \mathrm{~N}_{5} \mathrm{O}_{7} \mathrm{~S}\right)$ ESI m/z: calcd: 568.2235 [M+H] $]^{+}$, found: 568.2194; HPLC Rt: 10.907min.

(E)-6-Acetyl-7,9-dihydroxy-8,9b-dimethyl-2-(1-(((1-(2-oxo-2-(thiophen-2-yl)ethyl)-1H1,2,3-triazol-4-yl)methyl)amino)ethylidene)dibenzo[b,d]furan-1,3(2H,9bH)-dione $\quad(31)$ : The titled compound was synthesized according to the aforementioned general procedure

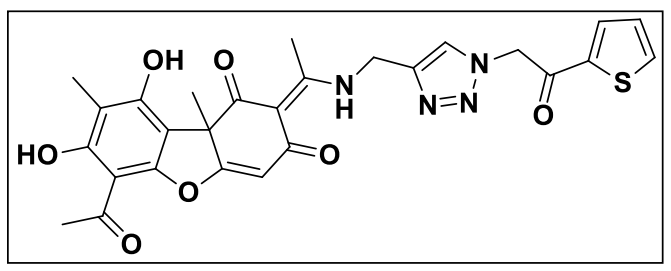

Method A by reacting alkyne 8 (100 $\mathrm{mg}, 0.26 \mathrm{mmol})$ with azide A22 (53 $\mathrm{mg}, 0.314 \mathrm{mmol})$. The product was obtained as colourless solid. Yield: $108 \mathrm{mg}$

(75\%); M.p.: 121-123 ${ }^{\circ} \mathrm{C}$; IR spectrum, $\left(v, \mathrm{~cm}^{-1}\right): 3446.23,3084.93,2926.68,1681.25$, $1625.23,1555.55,1464.56,1409.07,1368.77,1317.96,1284.31,1237.78,1188.43,1139.14$, 1059.10; ${ }^{1}$ H NMR (400 MHz, CDCl3): $\delta 13.90$ (s, 1H), 13.35 (s, 1H), 11.87 (s, 1H), 7.79 (s, 1H), $7.61(\mathrm{~d}, J=4.1 \mathrm{~Hz}, 1 \mathrm{H}), 7.20(\mathrm{~d}, J=4.1 \mathrm{~Hz}, 1 \mathrm{H}), 5.78(\mathrm{~s}, 1 \mathrm{H}), 5.71(\mathrm{~s}, 2 \mathrm{H}), 4.85(\mathrm{dd}, J=5.4$ \& $2.8 \mathrm{~Hz}, 2 \mathrm{H}), 3.70(\mathrm{~s}, 1 \mathrm{H}), 2.76(\mathrm{~s}, 3 \mathrm{H}), 2.67(\mathrm{~s}, 3 \mathrm{H}), 2.09(\mathrm{~s}, 3 \mathrm{H}), 1.70(\mathrm{~s}, 3 \mathrm{H}) ;{ }^{13} \mathbf{C ~ N M R}$ (101 MHz, CDCl3): $\delta$ 200.79, 198.66, 181.92, 163.66, 158.33, 155.98, 142.77, 141.68, 133.63, $132.06,125.92,123.97,108.22,105.12,101.52,55.02,39.65,32.09,31.40,18.77,7.63$; ESIMS: m/z $549.20[\mathrm{M}+\mathrm{H}]^{+}$; HRMS $\left(\mathrm{C}_{27} \mathrm{H}_{24} \mathrm{~N}_{4} \mathrm{O}_{7} \mathrm{~S}\right)$ ESI m/z: calcd: $549.1502[\mathrm{M}+\mathrm{H}]^{+}$, found: 549.1443; HPLC Rt: 17.233 min. 
(E)-6-Acetyl-2-(1-(((1-(2-(5-chlorothiophen-2-yl)-2-oxoethyl)-1H-1,2,3-triazol-4-

yl)methyl)amino)ethylidene)-7,9-dihydroxy-8,9b-dimethyldibenzo[b,d]furan-

1,3(2H,9bH)-dione (32): The titled compound was synthesized according to the

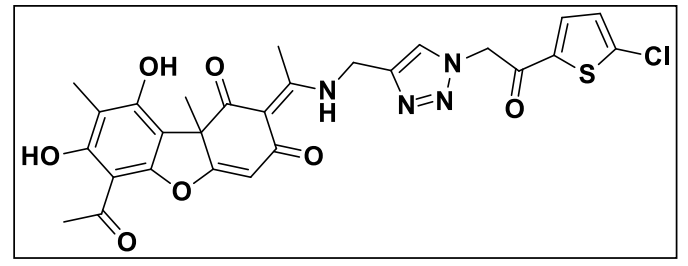
aforementioned general procedure Method A by reacting alkyne $\mathbf{8}(100 \mathrm{mg}, 0.26 \mathrm{mmol})$ with azide A23 (63.4 $\mathrm{mg}, 0.314 \mathrm{mmol})$. The product was obtained as colourless solid. Yield: $88 \mathrm{mg}(58 \%)$; M.p.: $121-123{ }^{\circ} \mathrm{C}$; IR spectrum, $\left(v, \mathrm{~cm}^{-1}\right)$ : $3436.53,3112.33,2927.37,2860.27,1687.53,1622.66,1556.26,1464.15,1417.47,1369.72$, 1282.70, 1238.59, 1189.19, 1134.29, 1059.92; ${ }^{1} \mathbf{H}$ NMR (400 MHz, CDCl $): \delta 13.88(\mathrm{~s}, 1 \mathrm{H})$, $13.34(\mathrm{~s}, 1 \mathrm{H}), 11.84(\mathrm{~s}, 1 \mathrm{H}), 7.79(\mathrm{~s}, 1 \mathrm{H}), 7.67$ (d, J=4.2 Hz, 1H), 7.05 (d, J=4.1 Hz, 1H), 5.77 (s, 1H), $5.72(\mathrm{~s}, 2 \mathrm{H}), 4.85(\mathrm{dd}, J=5.3 \& 2.6 \mathrm{~Hz}, 2 \mathrm{H}), 2.76(\mathrm{~s}, 3 \mathrm{H}), 2.66(\mathrm{~s}, 3 \mathrm{H}), 2.08(\mathrm{~s}, 3 \mathrm{H})$, 1.70 (s, 3H); ${ }^{13}$ C NMR (126 MHz, CDCl3): $\delta$ 200.80, 198.68, 182.09, 163.64, 158.33, 155.97, $142.77,138.78,133.12,128.41,123.97,108.21,105.11,101.51,54.92,39.64,32.08,31.42$, 18.79, 7.64; ESI-MS: m/z 583.15 [M+H] $]^{+}$; $\mathrm{HRMS}\left(\mathrm{C}_{27} \mathrm{H}_{23} \mathrm{CIN}_{4} \mathrm{O}_{7} \mathrm{~S}\right)$ ESI m/z: calcd: 583.1093 $[\mathrm{M}+\mathrm{H}]^{+}$, found: 583.1050; HPLC Rt: $10.444 \mathrm{~min}$.

(E)-6-Acetyl-2-(1-(((1-(2-(5-bromothiophen-2-yl)-2-oxoethyl)-1H-1,2,3-triazol-4yl)methyl)amino)ethylidene)-7,9-dihydroxy-8,9b-dimethyldibenzo[b,d]furan1,3(2H,9bH)-dione (33): The titled compound was synthesized according to the

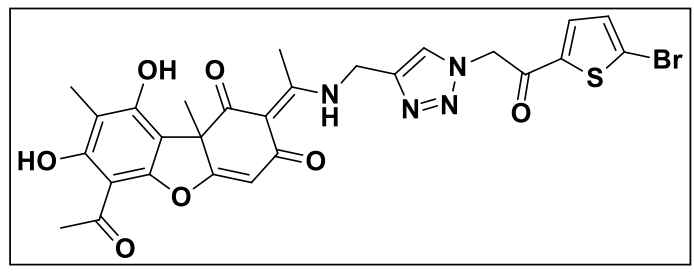
aforementioned general procedure Method A by reacting alkyne $\mathbf{8}(100 \mathrm{mg}, 0.26 \mathrm{mmol})$ with azide A24 $(78 \mathrm{mg}, 0.314 \mathrm{mmol})$. The product was obtained as colourless solid. Yield: $67 \mathrm{mg}(41 \%)$; M.p.: $125-127{ }^{\circ} \mathrm{C}$; IR spectrum, $\left(v, \mathrm{~cm}^{-1}\right)$ : $3446.46,2927.51,1678,1622.88,1556.63,1462.11,1410.72,1370.15,1325.11,1283.09$, 1237.44, 1190.10, 1132.89, 1061.70; ${ }^{1} \mathbf{H}$ NMR (400 MHz, CDCl3): $\delta 13.89$ (s, 1H), 13.35 (s, 1H), $11.86(\mathrm{~s}, 1 \mathrm{H}), 7.79$ (s, 1H), $7.61(\mathrm{~d}, J=4.1 \mathrm{~Hz}, 1 \mathrm{H}), 7.20$ (d, J=4.1 Hz, 1H), 5.77 (s, 1H), 
$5.71(\mathrm{~s}, 2 \mathrm{H}), 4.85(\mathrm{dd}, J=5.3 \& 2.8 \mathrm{~Hz}, 2 \mathrm{H}), 2.76(\mathrm{~s}, 3 \mathrm{H}), 2.67(\mathrm{~s}, 3 \mathrm{H}), 2.09(\mathrm{~s}, 3 \mathrm{H}), 1.70(\mathrm{~s}$, 3H); ${ }^{13}$ C NMR (101 MHz, CDCl $): \delta 200.80,198.66,181.93,163.64,158.32,155.96,142.76$, $141.66,133.63,132.06,125.92,123.98,108.20,105.11,101.51,55.01,39.64,32.08,31.42$, 29.83, 18.79, 7.64; ESI-MS: m/z $627.05[\mathrm{M}+\mathrm{H}]^{+}$; HRMS $\left(\mathrm{C}_{27} \mathrm{H}_{23} \mathrm{BrN}_{4} \mathrm{O}_{7} \mathrm{~S}\right)$ ESI m/z: calcd: $627.0587[\mathrm{M}+\mathrm{H}]^{+}$, found: 627.0544; HPLC Rt: $16.381 \mathrm{~min}$.

(E)-6-Acetyl-2-(1-(((1-(2-(benzo[b]thiophen-2-yl)-2-oxoethyl)-1H-1,2,3-triazol-4-yl) methyl)amino)ethylidene)-7,9-dihydroxy-8,9b-dimethyldibenzo[b,d]furan-1,3 $(2 \mathrm{H}, 9 b \mathrm{H})$ dione (34): The titled compound was synthesized according to the aforementioned general

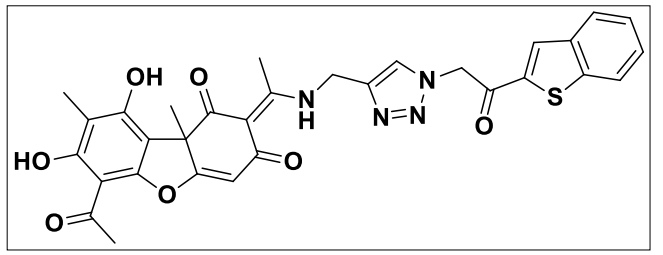
procedure Method B by reacting alkyne 8 (100 mg, $0.26 \mathrm{mmol})$ with azide $\mathbf{A 2 5}(68.3 \mathrm{mg}, 0.314 \mathrm{mmol})$. The product was obtained as colourless solid. Yield: 56 mg (31\%); M.p.: $123-125{ }^{\circ} \mathrm{C}$; IR spectrum, $\left(v, \mathrm{~cm}^{-1}\right): 3445.96,2926.66,2860.27,1690.44$, 1624.67, 1555.31, 1515.79, 1463.47, 1370.71, 1284.52, 1234.70, 1187.05, 1138.78, 1061.20; ${ }^{1}$ H NMR (400 MHz, CDCl) $) \delta 13.88$ (s, 1H), 13.35 (s, 1H), 11.84 (s, 1H), 8.13 (s, 1H), 7.95 (d, J=7.9 Hz, 1H), 7.89 (d, J=8.1 Hz, 1H), $7.85(\mathrm{~s}, 1 \mathrm{H}), 7.55-7.51(\mathrm{~m}, 1 \mathrm{H}), 7.47$ (dd, J=11.2 \& $4.0 \mathrm{~Hz}, 1 \mathrm{H}), 5.91(\mathrm{~s}, 2 \mathrm{H}), 5.76(\mathrm{~s}, 1 \mathrm{H}), 4.86(\mathrm{~d}, J=3.5 \mathrm{~Hz}, 2 \mathrm{H}), 2.76(\mathrm{~s}, 3 \mathrm{H}), 2.66(\mathrm{~s}, 3 \mathrm{H}), 2.09$

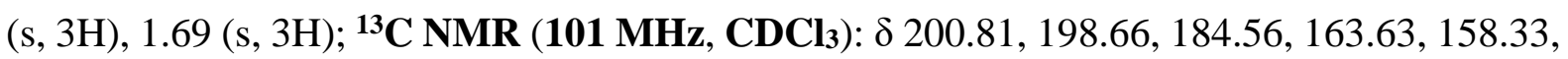
$155.96,143.08,142.76,139.48,138.85,130.85,128.73,126.61,125.79,124.07,123.20$, 108.21, 105.12, 101.51, 55.55, 39.68, 32.09, 31.43, 18.82, 7.64; ESI-MS: m/z 599.20 [M+H] ; HRMS $\left(\mathrm{C}_{31} \mathrm{H}_{26} \mathrm{~N}_{4} \mathrm{O}_{7} \mathrm{~S}\right)$ ESI m/z: calcd: 599.1624 [M+H] $]^{+}$, found: 599.1599; HPLC Rt: 11.024 $\min$. 
(E)-6-Acetyl-7,9-dihydroxy-8,9b-dimethyl-2-(1-(((1-(2-oxo-2-(thiazol-2-yl)ethyl)-1H-

1,2,3-triazol-4-yl)methyl)amino)ethylidene)dibenzo[b,d]furan-1,3(2H,9bH)-dione

(35):

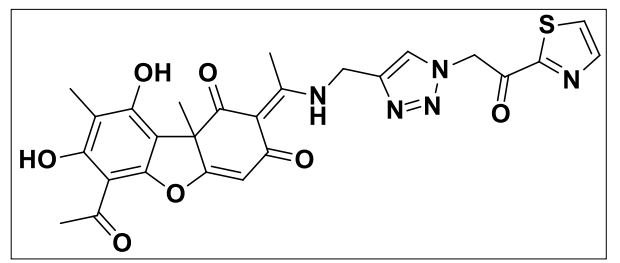

The titled compound was synthesized according to the aforementioned general procedure Method $\mathbf{A}$ by reacting alkyne 8 (100 $\mathrm{mg}, 0.26 \mathrm{mmol})$ with azide $\mathbf{A 2 6}$

(53 mg, $0.314 \mathrm{mmol}$ ). The product was obtained as colourless solid. Yield: $62 \mathrm{mg}$ (43\%); M.p.:

113-115 ${ }^{\circ} \mathrm{C}$; IR spectrum, $\left(v, \mathrm{~cm}^{-1}\right): 3448.78,2926.77,2849.32,1690.68,1624.83,1556.04$, 1463.93, 1410.74, 1370.11, 1320.06, 1284.24, 1238.04, 1189.65, 1138.87, 1062.46; ${ }^{1} \mathbf{H}$ NMR (500 MHz, CDCl $): \delta 13.88$ (s, 1H), 13.34 (s, 1H), 11.85 (s, 1H), 7.79 (s, 1H), 7.61 (d, J=4.1 Hz, 1H), $7.20(\mathrm{~d}, J=4.0 \mathrm{~Hz}, 1 \mathrm{H}), 5.77(\mathrm{~s}, 1 \mathrm{H}), 5.72(\mathrm{~s}, 2 \mathrm{H}), 4.85(\mathrm{~s}, 2 \mathrm{H}), 2.75(\mathrm{~s}, 3 \mathrm{H}), 2.66(\mathrm{~s}$, 3H), 2.08 (s, 3H), 1.70 (s, 3H); ${ }^{13}$ C NMR (101 MHz, CDCl3): $\delta$ 200.81, 198.65, 181.92, $163.64,158.32,155.97,141.65,133.64,132.06,125.94,123.99,108.21,105.11,101.51,55.01$, 39.64, 32.10, 31.43, 18.80, 7.64; ESI-MS: m/z 549.15 [M+H] $]^{+}$; HRMS $\left(\mathrm{C}_{26} \mathrm{H}_{23} \mathrm{~N}_{5} \mathrm{O}_{7} \mathrm{~S}\right)$ ESI m/z: calcd: $549.1484[\mathrm{M}+\mathrm{H}]^{+}$, found: 549.1462; HPLC Rt: $17.124 \mathrm{~min}$.

(E)-6-Acetyl-2-(1-(((1-(2-((1,1-dioxidobenzo[d]isothiazol-3-yl)oxy)ethyl)-1H-1,2,3triazol-4-yl)methyl)amino)ethylidene)-7,9-dihydroxy-8,9b-dimethyldibenzo[b,d]furan1,3(2H,9bH)-dione (36): The titled compound was synthesized according to the

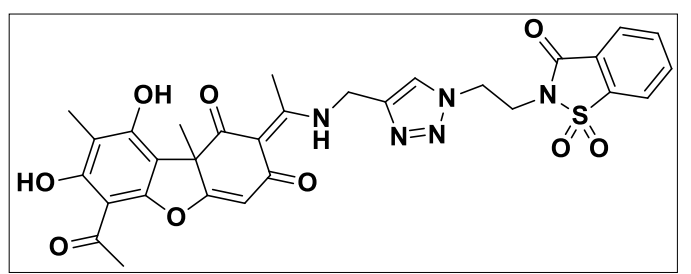
aforementioned general procedure Method A by reacting alkyne $\mathbf{8}(100 \mathrm{mg}, 0.26 \mathrm{mmol})$ with azide A27 (80 $\mathrm{mg}, 0.314 \mathrm{mmol})$. The product was obtained as colourless solid. Colourless solid, Yield: $152 \mathrm{mg}$ (92\%); M.p.: 147-149 ${ }^{\circ} \mathrm{C}$; IR spectrum, $\left(v, \mathrm{~cm}^{-1}\right): 3450.26,3095.89,2927.32,2854.79,1737.46,1699.64,1624.53,1556.43$, 1463.31, 1370.90, 1336.44, 1286.99, 1185.40, 1138.38, 1062.05; ${ }^{1}$ H NMR (400MHz, CDCl3): $\delta 13.80(\mathrm{~s}, 1 \mathrm{H}), 13.34(\mathrm{~s}, 1 \mathrm{H}), 11.87(\mathrm{~s}, 1 \mathrm{H}), 8.02(\mathrm{~d}, J=7.4 \mathrm{~Hz}, 1 \mathrm{H}), 7.92-7.80(\mathrm{~m}, 3 \mathrm{H}), 7.66$ (s, 1H), $5.74(\mathrm{~s}, 1 \mathrm{H}), 4.82(\mathrm{t}, J=6.0 \mathrm{~Hz}, 2 \mathrm{H}), 4.78(\mathrm{~d}, J=5.6 \mathrm{~Hz}, 2 \mathrm{H}), 4.28(\mathrm{t}, J=6.0 \mathrm{~Hz}, 2 \mathrm{H})$, 
2.66 (s, 6H), 2.08 (s, 3H), 1.67 (s, 3H); ${ }^{13}$ C NMR (126 MHz, CDCl $): \delta$ 200.79, 198.60, $175.70,163.63,158.76,158.32,155.96,137.50,135.42,134.83,126.76,125.72,122.94$, 121.30, 108.17, 105.11, 101.50, 48.20, 39.58, 38.91, 32.02, 31.41, 18.72, 7.64; ESI-MS: m/z $634.20[\mathrm{M}+\mathrm{H}]^{+}$; HRMS $\left(\mathrm{C}_{30} \mathrm{H}_{27} \mathrm{~N}_{5} \mathrm{O}_{9} \mathrm{~S}\right)$ ESI m/z: calcd: $634.1622[\mathrm{M}+\mathrm{H}]^{+}$, found: 634.1602; HPLC Rt: 11.521 min.

(E)-tert-Butyl-4-(2-(4-(((1-(6-acetyl-7,9-dihydroxy-8,9b-dimethyl-1,3-dioxo-1,9bdihydrodibenzo[b,d]furan-2(3H)-ylidene)ethyl)amino)methyl)-1H-1,2,3-triazol-1yl)acetyl)piperazine-1-carboxylate (37): The titled compound was synthesized according to

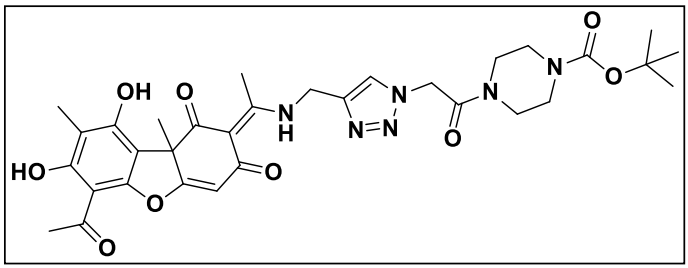
the aforementioned general procedure Method B by reacting alkyne $\mathbf{8}(100 \mathrm{mg}, 0.26 \mathrm{mmol})$ with azide $\mathbf{A 2 8}$ (85 mg, $0.314 \mathrm{mmol})$. The product was obtained as colourless solid. Yield: $100 \mathrm{mg}(59 \%)$; M.p.: $139-141{ }^{\circ} \mathrm{C}$; IR spectrum, $\left(v, \mathrm{~cm}^{-1}\right)$ : 3456.98, 2975.34, 2927.91, 2871.23, 1698.05, 1626.45, 1556.95, 1465.79, 1419.09, 1368.14, 1318.90, 1285.87, 1235.41, 1186.32, 1167.92, 1138.04, 1061.33; ${ }^{\mathbf{1}} \mathbf{H}$ NMR (400 $\mathbf{~ M H z ,}$ CDCl3): $\delta 13.86(\mathrm{~s}, 1 \mathrm{H}), 13.34(\mathrm{~s}, 1 \mathrm{H}), 11.86(\mathrm{~s}, 1 \mathrm{H}), 7.80(\mathrm{~s}, 1 \mathrm{H}), 5.77(\mathrm{~s}, 1 \mathrm{H}), 5.28(\mathrm{~s}, 2 \mathrm{H})$, $4.82(\mathrm{dd}, J=4.9 \& 3.1 \mathrm{~Hz}, 2 \mathrm{H}), 3.63-3.44(\mathrm{~m}, 8 \mathrm{H}), 2.75(\mathrm{~s}, 3 \mathrm{H}), 2.66(\mathrm{~s}, 3 \mathrm{H}), 2.08(\mathrm{~s}, 3 \mathrm{H}), 1.70$ (s, 3H), 1.47 (s, 9H); ${ }^{13} \mathbf{C}$ NMR (101 MHz, CDCl $): \delta 200.80,198.63,175.52,174.56,163.63$, $163.37,158.33,155.97,154.46,124.02,108.18,105.12,101.50,80.88,51.09,45.33,42.32$, 39.66, 32.08, 31.42, 28.47, 18.78, 7.63; ESI-MS: m/z $651.30[\mathrm{M}+\mathrm{H}]^{+} ; \mathrm{HRMS}\left(\mathrm{C}_{32} \mathrm{H}_{38} \mathrm{~N}_{6} \mathrm{O}_{9}\right)$ ESI m/z: calcd: $651.2799[\mathrm{M}+\mathrm{H}]^{+}$, found: 651.2780 ; HPLC Rt: $12.182 \mathrm{~min}$. 
(E)-6-Acetyl-7,9-dihydroxy-8,9b-dimethyl-2-(1-(((1-(2-oxo-2-(4-phenylpiperazin-1yl)ethyl)-1H-1,2,3-triazol-4-yl)methyl)amino)ethylidene)dibenzo[b,d]furan-1,3(2H,9bH)dione (38): The titled compound was synthesized according to the aforementioned general

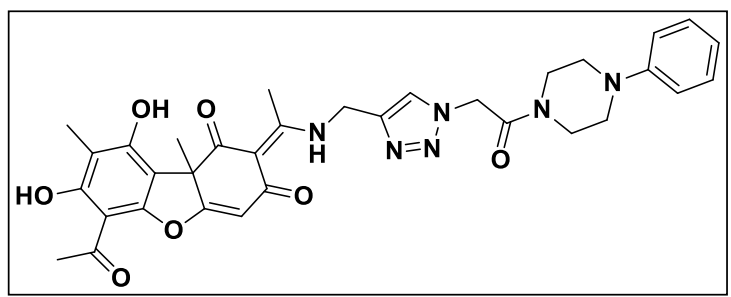
procedure Method B by reacting alkyne 8 (100 mg, 0.26 mmol) with azide $\mathbf{A 2 9}$ (77 $\mathrm{mg}, 0.314$ mmol). The product was obtained as colourless solid. Yield: $130 \mathrm{mg}(79 \%)$; M.p.: $107-109{ }^{\circ} \mathrm{C}$;

IR spectrum, $\left(v, \mathrm{~cm}^{-1}\right): 3448.64,2923.29,2106.14,1663.75,1623.92,1555.60,1461.64$, 1371.51, 1282.60, 1232.12, 1190.37, 1141.48, 1062.24; ${ }^{1} \mathbf{H}$ NMR (400 MHz, CDCl $): ~ \delta 13.85$ (s, 1H), $13.35(\mathrm{~s}, 1 \mathrm{H}), 11.86(\mathrm{~s}, 1 \mathrm{H}), 7.82(\mathrm{~s}, 1 \mathrm{H}), 7.30(\mathrm{dd}, J=10.8 \& 5.2 \mathrm{~Hz}, 1 \mathrm{H}), 6.93$ (d, $J=8.3 \mathrm{~Hz}, 4 \mathrm{H}), 5.76(\mathrm{~s}, 1 \mathrm{H}), 5.32(\mathrm{~s}, 2 \mathrm{H}), 4.82(\mathrm{~d}, J=2.3 \mathrm{~Hz}, 2 \mathrm{H}), 3.81-3.72(\mathrm{~m}, 4 \mathrm{H}), 3.25-3.18$ (m, 4H), 2.75 (s, 3H), 2.66 (s, 3H), 2.09 (s, 3H), 1.69 (s, 3H); ${ }^{\mathbf{3}} \mathbf{C}$ NMR (101 MHz, CDCl3): $\delta 200.81,198.63,163.63,163.19,158.33,155.97,150.65,142.50,129.48,124.03,121.20$, $117.00,108.19,105.13,101.51,51.10,49.81,49.44,45.49,42.49,39.67,32.09,31.42,18.80$, 7.64.; ESI-MS: m/z $627.30[\mathrm{M}+\mathrm{H}]^{+}$; HRMS $\left(\mathrm{C}_{33} \mathrm{H}_{34} \mathrm{~N}_{6} \mathrm{O}_{7}\right)$ ESI m/z: calcd: $627.2604[\mathrm{M}+\mathrm{H}]^{+}$, found: 627.2588; HPLC Rt: 13.217 min.

(E)-6-Acetyl-2-(1-(((1-(2-(4-(2-fluorophenyl)piperazin-1-yl)-2-oxoethyl)-1H-1,2,3-triazol4-yl)methyl)amino)ethylidene)-7,9-dihydroxy-8,9b-dimethyldibenzo[b,d]furan1,3(2H,9bH)-dione (39): The titled compound was synthesized according to the

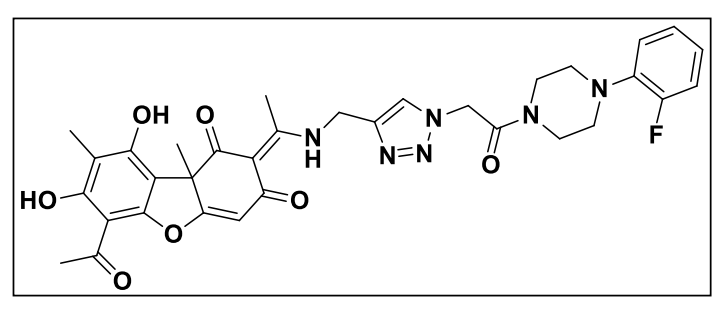
aforementioned general procedure Method B by reacting alkyne 8 (100 $\mathrm{mg}, 0.26 \mathrm{mmol})$ with azide A30 (74 $\mathrm{mg}, 0.314 \mathrm{mmol})$. The product was obtained as colourless solid. Yield: $71 \mathrm{mg}(42 \%)$; M.p.: $115-117{ }^{\circ} \mathrm{C}$; IR spectrum, $\left(v, \mathrm{~cm}^{-1}\right): 3145.21,2923.90,2854.79,1666.80,1623.90$, 1555.20, 1496.32, 1462.61, 1369.38, 1282.94, 1235.80, 1189.22, 1140.00, 1060.81; ${ }^{1}$ H NMR 
(400 MHz, CDCl3): $\delta 13.87$ (s, 1H), 13.35 (s, 1H), 11.87 (s, 1H), 7.82 (s, 1H), 7.10-6.91 (m, $4 \mathrm{H}), 5.77(\mathrm{~s}, 1 \mathrm{H}), 5.32(\mathrm{~s}, 2 \mathrm{H}), 4.88-4.78(\mathrm{~m}, 2 \mathrm{H}), 3.83-3.73(\mathrm{~m}, 4 \mathrm{H}), 3.15-3.08(\mathrm{~m}, 4 \mathrm{H}), 2.75$ (s, 3H), 2.66 (s, 3H), 2.09 (s, 3H), 1.70 (s, 3H); ${ }^{13} \mathbf{C}$ NMR (101 MHz, CDCl 3$): \delta 200.82$, $198.63,163.41\left(\mathrm{~d}, J_{\mathrm{C}-\mathrm{F}}=44.4 \mathrm{~Hz}\right), 158.35,155.93\left(\mathrm{t}, J_{\mathrm{C}-\mathrm{F}}=123.2 \mathrm{~Hz}\right), 142.50,139.22\left(\mathrm{~d}, J_{\mathrm{C}-}\right.$ $\mathrm{F}=8.6 \mathrm{~Hz}), 124.78\left(\mathrm{~d}, J_{\mathrm{C}-\mathrm{F}}=3.4 \mathrm{~Hz}\right), 124.06,123.76\left(\mathrm{~d}, J_{\mathrm{C}-\mathrm{F}}=8.1 \mathrm{~Hz}\right), 119.48,116.51\left(\mathrm{~d}, J_{\mathrm{C}-}\right.$ $\mathrm{F}=20.6 \mathrm{~Hz}), 108.19,105.14,101.51,51.08,50.54\left(\mathrm{~d}, J_{\mathrm{C}-\mathrm{F}}=56.0 \mathrm{~Hz}\right), 45.70,42.67,39.68,32.08$, 31.42, 18.80, 7.63; ESI-MS: m/z 645.25[M+H] $]^{+}$; HRMS $\left(\mathrm{C}_{33} \mathrm{H}_{33} \mathrm{FN}_{6} \mathrm{O}_{7}\right)$ ESI m/z: calcd: 645.2480 [M+H] $]^{+}$, found: 645.2469; HPLC Rt: 14.034 min.

(E)-6-Acetyl-2-(1-(((1-(2-(4-(2-chlorophenyl)piperazin-1-yl)-2-oxoethyl)-1H-1,2,3triazol-4-yl)methyl)amino)ethylidene)-7,9-dihydroxy-8,9b-dimethyldibenzo[ $[b, d]$ furan1,3(2H,9bH)-dione (40): The titled compound was synthesized according to the

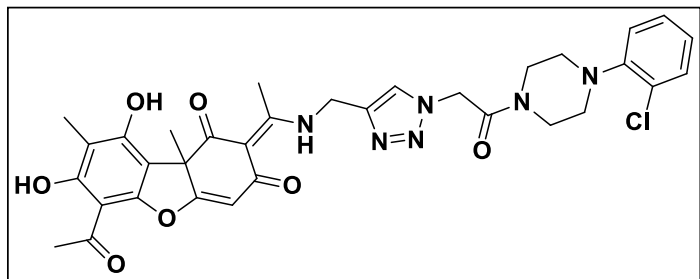
aforementioned general procedure Method B by reacting alkyne $\mathbf{8}(100 \mathrm{mg}, 0.26 \mathrm{mmol})$ with azide A31 (88 $\mathrm{mg}, 0.314 \mathrm{mmol})$. The product was obtained as colourless solid. Yield: $102 \mathrm{mg}(59 \%)$; M.p.: $141-143{ }^{\circ} \mathrm{C}$; IR spectrum, $\left(v, \mathrm{~cm}^{-1}\right)$ : $3451.02,2925.25,1748.67,1626.30,1555.24,1474.56,1371.22,1285.08,1229.85,1189.73$, 1140.95, 1066.41; ${ }^{1} \mathbf{H}$ NMR (500 MHz, CDCl): $\delta 13.87$ (s, 1H), 13.35 (s, 1H), 11.88 (s, 1H), $7.84(\mathrm{~s}, 1 \mathrm{H}), 7.39$ (dd, $J=7.9 \& 1.4 \mathrm{~Hz}, 1 \mathrm{H}), 7.24$ (dd, $J=7.7 \& 1.4 \mathrm{~Hz}, 1 \mathrm{H}), 7.03$ (ddd, $J=15.4$, $7.7 \& 1.3 \mathrm{~Hz}, 2 \mathrm{H}), 5.78(\mathrm{~s}, 1 \mathrm{H}), 5.33(\mathrm{~s}, 2 \mathrm{H}), 4.84(\mathrm{~s}, 2 \mathrm{H}), 3.84-3.74(\mathrm{~m}, 4 \mathrm{H}), 3.12-3.05(\mathrm{~m}$, 4H), 2.76 (s, 3H), 2.67 (s, 3H), 2.09 (s, 3H), 1.70 (s, 3H); $\left.{ }^{13} \mathbf{C ~ N M R ~ ( 1 0 1 ~ M H z , ~ C D C l ~}\right)$ ): $\delta$ 200.81, 198.63, 163.62, 163.28, 158.34, 155.97, 148.28, 130.93, 129.11, 128.04, 124.80, $124.12,120.70,108.18,105.14,101.51,51.45,51.14,50.90,45.84,42.85,39.69,32.09,31.42$, 18.80, 7.63; ESI-MS: m/z 661.25 [M+H] $]^{+}$; HRMS $\left(\mathrm{C}_{33} \mathrm{H}_{33} \mathrm{ClN}_{6} \mathrm{O}_{7}\right)$ ESI m/z: calcd: 661.2195 $[\mathrm{M}+\mathrm{H}]^{+}$, found: 661.2170; HPLC Rt: $10.493 \mathrm{~min}$. 
(E)-6-Acetyl-7,9-dihydroxy-8,9b-dimethyl-2-(1-(((1-(2-oxo-2-(4-(4-(trifluoromethyl) phenyl)piperazin-1-yl)ethyl)-1H-1,2,3-triazol-4-yl)methyl)amino)ethylidene)dibenzo $[\boldsymbol{b}, \boldsymbol{d}]$ furan-1,3(2H,9bH)-dione (41): The titled compound was synthesized according to the

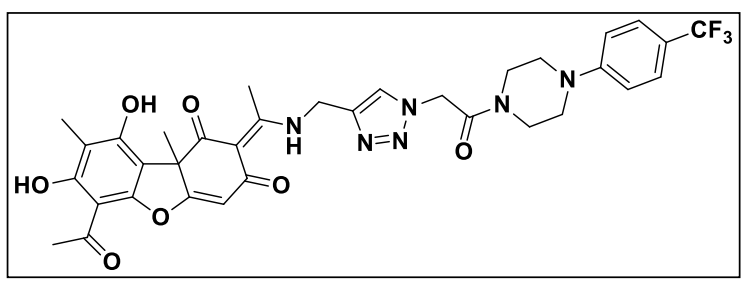
aforementioned general procedure Method B by reacting alkyne $8(100 \mathrm{mg}, 0.26 \mathrm{mmol})$ with azide A32 (98.5 mg, $0.314 \mathrm{mmol})$. The product was obtained as colourless solid. Yield: $127 \mathrm{mg}(70 \%)$; M.p.: $127-129^{\circ} \mathrm{C}$; IR spectrum, $\left(v, \mathrm{~cm}^{-}\right.$ $\left.{ }^{1}\right): 3448.78,2926.03,2362.50,2107.63,1665.47,1619.64,1556.65,1465.78,1372.19$, $1330.76,1284.43,1232.47,1190.29,1160.50,1140.48,1114.89,1069.45 ;{ }^{1}$ H NMR (400 MHz, CDCl $): \delta 13.90$ (s, 1H), 13.35 (s, 1H), 11.86 (s, 1H), 7.85 (s, 1H), 7.52 (d, J=7.9 Hz, 2H), $6.94(\mathrm{~d}, J=7.9 \mathrm{~Hz}, 2 \mathrm{H}), 5.76(\mathrm{~s}, 1 \mathrm{H}), 5.34(\mathrm{~s}, 2 \mathrm{H}), 4.83(\mathrm{~s}, 2 \mathrm{H}), 3.79(\mathrm{~d}, J=18.9 \mathrm{~Hz}, 4 \mathrm{H})$, $3.33(\mathrm{~d}, J=21.5 \mathrm{~Hz}, 4 \mathrm{H}), 2.76(\mathrm{~s}, 3 \mathrm{H}), 2.66(\mathrm{~s}, 3 \mathrm{H}), 2.09(\mathrm{~s}, 3 \mathrm{H}), 1.70(\mathrm{~s}, 3 \mathrm{H}) ;{ }^{\mathbf{1 3}} \mathbf{C} \mathbf{N M R}(\mathbf{1 0 1}$ MHz, CDCl $): \delta 200.81,198.68,163.47\left(\mathrm{~d}, J_{\mathrm{C}-\mathrm{F}}=33.7 \mathrm{~Hz}\right), 157.14\left(\mathrm{~d}, J_{\mathrm{C}-\mathrm{F}}=235.7 \mathrm{~Hz}\right), 152.67$, $126.74\left(\mathrm{~d}, J_{\mathrm{C}-\mathrm{F}}=3.2 \mathrm{~Hz}\right), 122.06\left(\mathrm{~d}, J_{\mathrm{C}-\mathrm{F}}=32.7 \mathrm{~Hz}\right), 115.44,108.18,105.11,101.51,51.17,48.30$ $\left(\mathrm{d}, J_{\mathrm{C}-\mathrm{F}}=34.1 \mathrm{~Hz}\right), 45.26,42.13,39.71,32.08,31.40,18.79,7.63 ;$ ESI-MS: m/z 695.25 [M+H] ${ }^{+}$; HRMS ( $\left.\mathrm{C}_{34} \mathrm{H}_{33} \mathrm{~F}_{3} \mathrm{~N}_{6} \mathrm{O}_{7}\right)$ ESI m/z: calcd: 695.2470 [M+H] $]^{+}$, found: 695.2442; HPLC Rt: 16.228 $\min$.

(E)-6-Acetyl-7,9-dihydroxy-2-(1-(((1-(2-(4-(2-methoxyphenyl)piperazin-1-yl)-2oxoethyl)-1H-1,2,3-triazol-4-yl)methyl)amino)ethylidene)-8,9bdimethyldibenzo $[\boldsymbol{b}, \boldsymbol{d}]$ furan-1,3(2H,9bH)-dione (42): The titled compound was synthesized

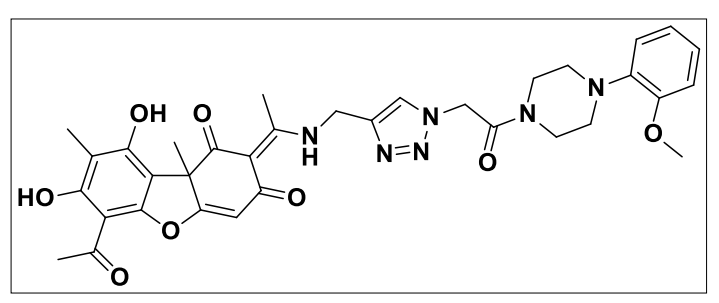
according to the aforementioned general procedure Method B by reacting alkyne 8 (100 $\mathrm{mg}, 0.26 \mathrm{mmol}$ ) with azide $\mathbf{A 3 3}(87 \mathrm{mg}, 0.314$ mmol). The product was obtained as colourless solid. Yield: $150 \mathrm{mg}$ (87\%); M.p.: $103-105{ }^{\circ} \mathrm{C}$; IR spectrum, $\left(v, \mathrm{~cm}^{-1}\right): 3465.87,2924.50$, 
$2832.88,2105.60,1664.04,1624.81,1555.23,1495.25,1462.05,1370.34,1282.39,1239.13$, 1186.88, 1142.13, 1060.20, 1030.00; ${ }^{\mathbf{1}} \mathbf{H}$ NMR (500 MHz, CDCl3): $\delta 13.86$ (s, 1H), 13.35 (s, 1H), $11.88(\mathrm{~s}, 1 \mathrm{H}), 7.84(\mathrm{~s}, 1 \mathrm{H}), 7.07-7.04(\mathrm{~m}, 1 \mathrm{H}), 6.95-6.89(\mathrm{~s}, 3 \mathrm{H}), 5.78(\mathrm{~s}, 1 \mathrm{H}), 5.32(\mathrm{~s}$, 2H), $4.84(\mathrm{~s}, 2 \mathrm{H}), 3.88(\mathrm{~s}, 3 \mathrm{H}), 3.84-3.82(\mathrm{~m}, 2 \mathrm{H}), 3.74-3.73(\mathrm{~m}, 2 \mathrm{H}), 3.12-3.11(\mathrm{~m}, 2 \mathrm{H}), 3.08-$ $3.06(\mathrm{~m}, 2 \mathrm{H}), 2.76(\mathrm{~s}, 3 \mathrm{H}), 2.67(\mathrm{~s}, 3 \mathrm{H}), 2.09(\mathrm{~s}, 3 \mathrm{H}), 1.70(\mathrm{~s}, 3 \mathrm{H}) ;{ }^{13} \mathbf{C}$ NMR (101 MHz,

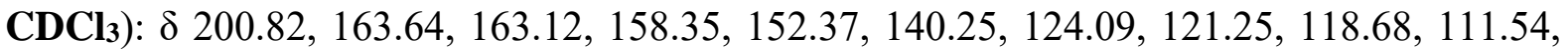
$108.20,105.16,101.52,55.61,51.13,50.88,50.41,45.83,42.82,39.70,32.08,31.43,18.81$, 7.63; ESI-MS: m/z $657.25[\mathrm{M}+\mathrm{H}]^{+}$; HRMS $\left(\mathrm{C}_{34} \mathrm{H}_{36} \mathrm{~N}_{6} \mathrm{O}_{8}\right)$ ESI m/z: calcd: $657.2703[\mathrm{M}+\mathrm{H}]^{+}$, found: 657.2689; HPLC Rt: 9.227 min.

(E)-6-Acetyl-2-(1-(((1-(2-(4-cinnamylpiperazin-1-yl)-2-oxoethyl)-1H-1,2,3-triazol-4yl)methyl)amino)ethylidene)-7,9-dihydroxy-8,9b-dimethyldibenzo[b,d]furan1,3(2H,9bH)-dione (43): The titled compound was synthesized according to the

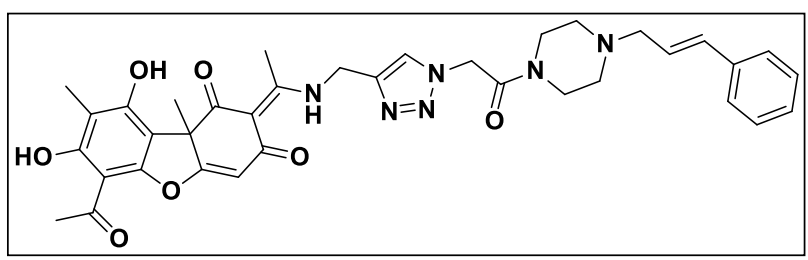
aforementioned general procedure Method B by reacting alkyne $\mathbf{8}(100 \mathrm{mg}, 0.26 \mathrm{mmol})$ with azide $\mathbf{A} 34$ (90 $\mathrm{mg}, 0.314 \mathrm{mmol})$. The product was obtained as colourless solid. Yield: 95 mg (52\%); M.p.: 110-112 ${ }^{\circ}$ C; IR spectrum, $\left(v, \mathrm{~cm}^{-1}\right): 3448.80,2925.06,2810.96,1698.84,1626.50,1556.77,1465.85,1421.41,1370.06$,

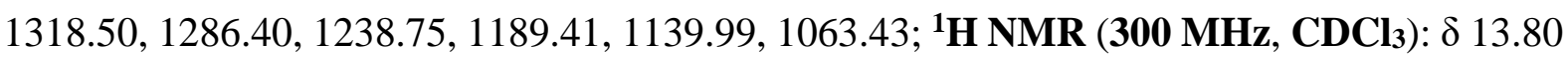
(s, 1H), $13.36(\mathrm{~s}, 1 \mathrm{H}), 11.86(\mathrm{~s}, 1 \mathrm{H}), 7.80(\mathrm{~s}, 1 \mathrm{H}), 7.30$ (ddd, J=29.4, 14.5 \& $6.9 \mathrm{~Hz}, 5 \mathrm{H}), 6.54$ $(\mathrm{d}, J=15.9 \mathrm{~Hz}, 1 \mathrm{H}), 6.27-6.18(\mathrm{~m}, 1 \mathrm{H}), 5.77(\mathrm{~s}, 1 \mathrm{H}), 5.27(\mathrm{~s}, 2 \mathrm{H}), 4.82(\mathrm{~d}, J=4.3 \mathrm{~Hz}, 2 \mathrm{H})$, 3.67-3.59 (2s, 4H), 3.19 (d, J=6.5 Hz, 2H), 2.74 (s, 3H), 2.66 (s, 3H), 2.52 (s, 4H), 2.09 (s, 3H), 1.69 (s, 3H); ${ }^{13}$ C NMR (126 MHz, CDCl3): $\delta$ 200.76, 198.54, 175.46, 163.57, 163.04, 158.29, $155.93,142.34,136.61,133.91,133.79,128.72,127.87,127.82,126.43,125.66,125.50$, $124.08,108.08,105.10,102.58,101.44,60.89,60.80,60.49,53.01,52.87,52.70,52.49,51.02$, $50.74,45.38,45.15,42.49,42.14,39.62,32.06,31.37,21.15,18.74,14.30,7.61 ;$ ESI-MS: m/z 
$667.35[\mathrm{M}+\mathrm{H}]^{+}$; HRMS $\left(\mathrm{C}_{36} \mathrm{H}_{38} \mathrm{~N}_{6} \mathrm{O}_{7}\right)$ ESI m/z: calcd: $667.2925[\mathrm{M}+\mathrm{H}]^{+}$, found: 667.2897 ; HPLC Rt: 15.806 min.

(E)-6-Acetyl-7,9-dihydroxy-8,9b-dimethyl-2-(1-(((1-(2-oxo-2-(4-(pyridin-2-yl)piperazin1-yl)ethyl)-1H-1,2,3-triazol-4-yl)methyl)amino)ethylidene)dibenzo[b,d]furan-

1,3(2H,9bH)-dione (44): The titled compound was synthesized according to the

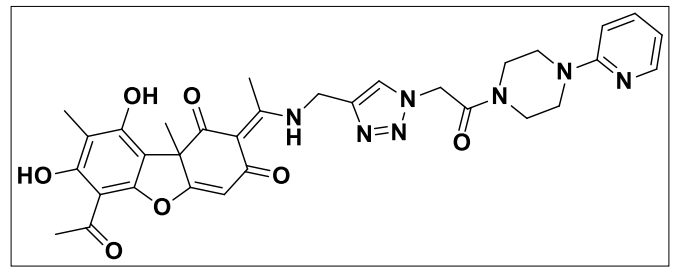
aforementioned general procedure Method B by reacting alkyne $\mathbf{8}(100 \mathrm{mg}, 0.26 \mathrm{mmol})$ with azide A35 (77.4 mg, $0.314 \mathrm{mmol})$. The product was obtained as colourless solid. Yield: $18 \mathrm{mg}(11 \%)$; M.p.: $115-117{ }^{\circ} \mathrm{C}$; IR spectrum, $\left(v, \mathrm{~cm}^{-1}\right)$ : $3450.48,2923.38,2105.82,1623.73,1555.21,1473.60,1369.99,1280.99,1234.47,1188.45$, 1133.07, 1059.53; ${ }^{1} \mathrm{H}$ NMR (400 MHz, CDCl $)$ ) $\delta 13.88$ (s, 1H), 13.35 (s, 1H), 11.88 (s, 1H), $8.20(\mathrm{~d}, J=4.3 \mathrm{~Hz}, 1 \mathrm{H}), 7.82(\mathrm{~s}, 1 \mathrm{H}), 7.52$ (t, $J=7.8 \mathrm{~Hz}, 1 \mathrm{H}), 6.71-6.65$ (m, 2H), 5.76 (s, 1H), $5.32(\mathrm{~s}, 2 \mathrm{H}), 4.82(\mathrm{~s}, 2 \mathrm{H}), 3.77(\mathrm{~s}, 2 \mathrm{H}), 3.69(\mathrm{~s}, 4 \mathrm{H}), 3.55(\mathrm{~s}, 2 \mathrm{H}), 2.75(\mathrm{~s}, 3 \mathrm{H}), 2.66(\mathrm{~s}, 3 \mathrm{H})$, 2.09 (s, 3H), 1.69 (s, 3H); ${ }^{13}$ C NMR (101 MHz, CDCl $): \delta 200.80,198.60,175.50,163.62$, $163.36,158.82,158.33,155.96,148.17,142.47,137.94,124.04,114.48,108.16,107.44$, 105.13, 101.49, 51.13, 45.19, 45.05, 42.11, 39.67, 32.08, 31.41, 18.78, 7.62.; ESI-MS: m/z 628.25[M+H $]^{+}$; HRMS $\left(\mathrm{C}_{32} \mathrm{H}_{33} \mathrm{~N}_{7} \mathrm{O}_{7}\right)$ ESI m/z: calcd: $628.2569[\mathrm{M}+\mathrm{H}]^{+}$, found: 628.2541 ; HPLC Rt: 10.367 min.

\section{BIOLOGY}

In vitro Mtb MABA Assay. ${ }^{9}$ Briefly, the inoculum was prepared from fresh LJ medium resuspended in 7H9-S medium (7H9 broth, $0.1 \%$ casitone, $0.5 \%$ glycerol, supplemented oleic acid, albumin, dextrose, and catalase [OADC]), adjusted to $\mathrm{OD}_{590} 1.0$, and diluted 1:20; 100 $\mu \mathrm{L}$ was used as inoculum. Each drug stock solution was thawed and diluted in 7H9-S at fourfold the final highest concentration tested. Serial two-fold dilutions of each drug were prepared directly in a sterile 96-well microtiter plate using $100 \mu \mathrm{L}$ 7H9-S. A growth control containing 
no antibiotic and a sterile control were also prepared on each plate. Sterile water was added to all perimetre wells to avoid evaporation during the incubation. The plate was covered, sealed in plastic bags and incubated at $37^{\circ} \mathrm{C}$ in normal atmosphere. After 7 days incubation, $30 \mu \mathrm{L}$ of alamar blue solution was added to each well, and the plate was re-incubated overnight. A change in colour from blue (oxidised state) to pink (reduced) indicated the growth of bacteria, and the MIC was defined as the lowest concentration of drug that prevented this change in colour.

Nutrient Starvation Model. ${ }^{10}$ A culture of M. tuberculosis H37Rv (O.D. of 0.8 - 1.0) grown in Middlebrook 7H9 medium supplemented with OADC was pelleted and washed twice with PBS. The pellet was resuspended in PBS in sealed bottles and incubated at $37{ }^{\circ} \mathrm{C}$ for 6 weeks. Aliquots of these cultures were then treated with standard drugs like INH, Rif and Ethambutol the lead compounds for 7 days at a concentration of $10 \mu \mathrm{g} / \mathrm{mL}$. The frequency of persistors was enumerated by MPN assay.

In vitro Antibacterial Activity. ${ }^{11}$ In vitro antibacterial activity of the newly synthesized compounds was studied against the bacterial strains, Bacillus subtilis (MTCC 441), Staphylococcus aureus (MTCC 96), Staphylococcus epidermidis and gram-negative organisms viz Escherichia coli (MTCC 443), Pseudomonas aeruginosa (MTCC 741) and Klebsiella pneumoniae (MTCC 618) by Agar Well Diffusion Method. The Nutrient Agar (NA) medium was suspended in distilled water $(28 \mathrm{~g}$ in $1000 \mathrm{~mL})$ and heated to boiling until it dissolved completely, the medium and Petri dishes were autoclaved at pressure of $15 \mathrm{lb} / \mathrm{inc}^{2}$ for $20 \mathrm{~min}$. Agar well bioassay was employed for testing antibacterial activity. The medium was poured into sterile petri dishes under aseptic conditions in a laminar air flow chamber. When the medium in the plates solidified, $0.5 \mathrm{~mL}$ of (week old) culture of test organism was inoculated and uniformly spread over the agar surface with a sterile L-shaped rod. Solutions were prepared by dissolving the compound in DMSO and $200 \mu \mathrm{g} / \mathrm{mL}$ concentrations were made. After 
inoculation, wells were scooped out with $3 \mathrm{~mm}$ sterile cork borer and the lids of the dishes were replaced. To each well different concentrations of test solutions were added. Controls were maintained. The treated and the controls were kept at $37^{\circ} \mathrm{C}$ for $24 \mathrm{~h}$ incubator. Inhibition zones were measured, the diameter calculated in millimeter and the corresponding results were tabulated. Following the zone of inhibition assay, the minimum inhibitory concentration was determined for all the synthesized compounds.

Table S3. Antibacterial Activity of Compounds 10-44 by Agar Well Diffusion Method (Zone of Inhibition in $\mathrm{mm}$ )

\begin{tabular}{|c|c|c|c|c|c|c|}
\hline Compound & S.aureus & S.epidermidis & $\begin{array}{c}B . \\
\text { subtilis }\end{array}$ & E.coli & P.aeruginosa & K.pneumoniae \\
\hline 10 & - & - & - & - & - & - \\
\hline 11 & - & - & - & - & - & - \\
\hline 12 & - & - & - & - & - & - \\
\hline 13 & - & - & - & - & - & - \\
\hline 14 & - & - & - & - & - & - \\
\hline 15 & - & - & - & - & - & - \\
\hline 16 & - & - & 12 & - & - & - \\
\hline 17 & - & - & 12 & - & - & - \\
\hline $18 * *$ & - & - & 15 & - & - & - \\
\hline 19 & - & - & - & - & - & - \\
\hline 20 & - & - & - & - & - & - \\
\hline 21 & - & - & - & - & - & - \\
\hline 22 & - & - & - & - & - & - \\
\hline 23 & - & - & - & - & - & - \\
\hline 24 & - & - & - & - & - & - \\
\hline 25 & - & - & - & - & - & - \\
\hline 26 & - & - & - & - & - & - \\
\hline 27 & - & - & 11 & - & - & - \\
\hline 28 & - & - & - & - & - & - \\
\hline $29 *$ & - & - & 13 & - & - & - \\
\hline 30 & - & - & - & - & - & - \\
\hline 31 & - & - & - & - & - & - \\
\hline 32 & - & - & - & - & - & - \\
\hline 33 & - & - & - & - & - & - \\
\hline 34 & - & - & - & - & - & - \\
\hline 35 & - & - & - & - & - & - \\
\hline 36 & - & - & - & - & - & - \\
\hline 37 & - & - & - & - & - & - \\
\hline 38 & - & - & - & - & - & - \\
\hline
\end{tabular}




\begin{tabular}{|c|c|c|c|c|c|c|}
\hline 39 & - & - & - & - & - & - \\
\hline $\mathbf{4 0}$ & - & - & - & - & - & - \\
\hline $\mathbf{4 1}$ & - & - & - & - & - & - \\
\hline $\mathbf{4 2}$ & - & - & $\mathbf{1 0}$ & - & - & - \\
\hline $\mathbf{4 3}$ & - & - & - & - & - & - \\
\hline $\mathbf{4 4}$ & - & - & - & - & - & - \\
\hline Penicillin & 28 & 26 & 28 & 24 & 23 & 25 \\
\hline Streptomycin & 23 & 25 & 24 & 23 & 28 & 26 \\
\hline
\end{tabular}

* and ** - compounds $29 \& 18$ are labeled as compound $\mathbf{5}$, and $\mathbf{6}$ respectively, while performing the study. S.aureus - Staphylococcus aureus; S.epidermis - Staphylococcus epidermis; B.subtilis - Bacillus subtilis; E.coli - Escherichia coli; P.aeruginosa - Pseudomonas aeruginosa; K.pneumoniae Klebsiella pneumoniae; (-) No activity.

Determination of Minimum Inhibitory Concentration. ${ }^{12}$ The minimum inhibitory concentrations (MIC) of various synthetic compounds were tested against three representative Gram-positive organisms viz. Bacillus subtilis (MTCC 441), Staphylococcus aureus (MTCC 96), Staphylococcus epidermidis and Gram-negative organisms viz Escherichia coli (MTCC 443), Pseudomonas aeruginosa (MTCC 741) and Klebsiella pneumoniae (MTCC 618) by Microdilution method recommended by CLSI Standard Protocol in liquid medium (Nutrient agar) distributed in 96-well plates.Serial dilutions of the tested compounds were performed (concentrations from $200 \mu \mathrm{g} / \mathrm{mL}$ to $1.56 \mu \mathrm{g} / \mathrm{mL}$ ) in a $200 \mu \mathrm{L}$ culture medium final volume; afterwards each well was seeded with a $50 \mu \mathrm{L}$ microbial suspension of 0.5 MacFarland densities. In each test, a microbial culture control and a sterility control (negative) were performed. The plates were incubated for 24 hours at $37{ }^{\circ} \mathrm{C}$. The lowest concentration which inhibited the visible microbial growth was considered the MIC $(\mu \mathrm{g} / \mathrm{mL})$ value for the tested compound. Penicillin and Streptomycin were used as standard drugs. The MIC values are expressed in $\mu \mathrm{M}$ units.

Table S4. Antibacterial Activity (MIC $\mu \mathrm{M}$ ) Compounds 10-44

\begin{tabular}{|c|c|c|c|c|c|c|}
\hline Compound & S.aureus & S.epidermidis & B.subtilis & E.coli & P.aeruginosa & K.pneumoniae \\
\hline 10 & $>350$ & $>350$ & $>350$ & $>350$ & $>350$ & $>350$ \\
\hline
\end{tabular}




\begin{tabular}{|c|c|c|c|c|c|c|}
\hline 11 & $>350$ & $>350$ & $>350$ & $>350$ & $>350$ & $>350$ \\
\hline 12 & $>350$ & $>350$ & $>350$ & $>350$ & $>350$ & $>350$ \\
\hline 13 & $>350$ & $>350$ & $>350$ & $>350$ & $>350$ & $>350$ \\
\hline 14 & $>350$ & $>350$ & $>350$ & $>350$ & $>350$ & $>350$ \\
\hline 15 & $>350$ & $>350$ & 346.8 & $>350$ & $>350$ & $>350$ \\
\hline 16 & $>350$ & $>350$ & 172.8 & $>350$ & $>350$ & $>350$ \\
\hline 17 & $>350$ & $>350$ & 158.1 & $>350$ & $>350$ & $>350$ \\
\hline 18 & $>350$ & $>350$ & 40.9 & $>350$ & $>350$ & $>350$ \\
\hline 19 & $>350$ & $>350$ & $>350$ & $>350$ & $>350$ & $>350$ \\
\hline 20 & $>350$ & $>350$ & $>350$ & $>350$ & $>350$ & $>350$ \\
\hline 21 & $>350$ & $>350$ & $>350$ & $>350$ & $>350$ & $>350$ \\
\hline 22 & $>350$ & $>350$ & $>350$ & $>350$ & $>350$ & $>350$ \\
\hline 23 & $>350$ & $>350$ & $>350$ & $>350$ & $>350$ & $>350$ \\
\hline 24 & $>350$ & $>350$ & $>350$ & $>350$ & $>350$ & $>350$ \\
\hline 25 & $>350$ & $>350$ & $>350$ & $>350$ & $>350$ & $>350$ \\
\hline 26 & $>350$ & $>350$ & $>350$ & $>350$ & $>350$ & $>350$ \\
\hline 27 & $>350$ & $>350$ & 168.7 & $>350$ & $>350$ & $>350$ \\
\hline 28 & $>350$ & $>350$ & $>350$ & $>350$ & $>350$ & $>350$ \\
\hline 29 & $>350$ & $>350$ & 90.7 & $>350$ & $>350$ & $>350$ \\
\hline 30 & $>350$ & $>350$ & $>350$ & $>350$ & $>350$ & $>350$ \\
\hline 31 & $>350$ & $>350$ & $>350$ & $>350$ & $>350$ & $>350$ \\
\hline 32 & $>350$ & $>350$ & $>350$ & $>350$ & $>350$ & $>350$ \\
\hline 33 & $>350$ & $>350$ & $>350$ & $>350$ & $>350$ & $>350$ \\
\hline 34 & $>350$ & $>350$ & $>350$ & $>350$ & $>350$ & $>350$ \\
\hline 35 & $>350$ & $>350$ & $>350$ & $>350$ & $>350$ & $>350$ \\
\hline 36 & $>350$ & $>350$ & $>350$ & $>350$ & $>350$ & $>350$ \\
\hline 37 & $>350$ & $>350$ & $>350$ & $>350$ & $>350$ & $>350$ \\
\hline 38 & $>350$ & $>350$ & $>350$ & $>350$ & $>350$ & $>350$ \\
\hline 39 & $>350$ & $>350$ & $>350$ & $>350$ & $>350$ & $>350$ \\
\hline 40 & $>350$ & $>350$ & 302.5 & $>350$ & $>350$ & $>350$ \\
\hline 41 & $>350$ & $>350$ & $>350$ & $>350$ & $>350$ & $>350$ \\
\hline 42 & $>350$ & $>350$ & 304.6 & $>350$ & $>350$ & $>350$ \\
\hline 43 & $>350$ & $>350$ & $>350$ & $>350$ & $>350$ & $>350$ \\
\hline 44 & $>350$ & $>350$ & $>350$ & $>350$ & $>350$ & $>350$ \\
\hline Penicillin & 3.5 & 7.0 & 3.5 & 27.8 & 27.8 & 27.8 \\
\hline Streptomycin & 4.0 & 4.0 & 8.1 & 8.1 & 2.0 & 8.1 \\
\hline
\end{tabular}

In vitro Cytotoxicity Evaluation. Cytotoxicity assay (MTT) on normal cells evaluated for all the test compounds were followed as per our established protocols. ${ }^{13}$ Two cell lines viz., HEK293: Homo sapiens embryonic kidney cells (ATCC® CRL-1573 ${ }^{\mathrm{TM}}$ ) and CHO-K1: Chinese hamster ovary cells, (ATCC® CCL-61 ${ }^{\mathrm{TM}}$ ) were obtained from the ATCC (Bethesda, MD, 
USA) and maintained in DMEM supplemented with $10 \%$ FBS, 2 mM l-glutamine, $100 \mathrm{U} / \mathrm{mL}$ penicillin, and $100 \mu \mathrm{g} / \mathrm{mL}$ streptomycin at $37{ }^{\circ} \mathrm{C}$ in a $5 \% \mathrm{CO}_{2}$ incubator. After seeding of cells in 96 well culture plate, the cells were allowed to attach properly. Test compounds of different concentrations ranging from 1 to $100 \mu \mathrm{M}$ were added in triplicates and incubated for $24 \mathrm{hr}$. The cells were then incubated with MTT $(0.5 \mathrm{mg} / \mathrm{mL})$ for $3 \mathrm{hr}$. To dissolve the insoluble formazan crystals, $100 \mu \mathrm{L}$ DMSO was added to each well and finally the absorbance of the plates were measured using a Synergy H1 multi-mode plate reader, USA. Mitomycin C was used as positive control for the comparison.

Molecular Docking. In silico analysis was performed in Dell Precision T7610 workstation (8 processors; 8 GB RAM; NVIDIA 3GB graphics; Maestro 9.8, Schrodinger, New York, U.S.A) workstation running on Redhat 6.1 Linux environment.The structure of ligand was drawn in ChemDraw Ultra 6.0. Molecular docking studies were performed against Mycobacterium tuberculosis enzyme enoyl reductase (InhA) (PDB - 4TZK). ${ }^{14,15}$ The protein was prepared with the help of Protein Preparation Wizard of Schrödinger Suite $9.8,{ }^{16 a}$ and optimized and minimized using OPLS_2005 (optimized potential for liquid simulations) force field algorithm.The grid generationwas performed utilizing the Glide Grid Generation panel. The co-crystallized inhibitor 1-cyclohexyl- $N$-(3,5-dichlorophenyl)-5-oxopyrrolidine-3carboxamide and test compounds were energy minimized using LigPrep module ${ }^{16 \mathrm{~b}}$ in Schrodinger. The minimized test compounds were docked using Glide XP docking calculations. The XP Glide scoring function was used to get the best ranked compounds and the specific interactions like H-bonds, $\pi$-cation and $\pi$ - $\pi$ stacking and van der Waals were analyzed using XP visualizer in Glide module. ${ }^{16 \mathrm{c}}$ 
${ }^{1} \mathrm{H} \&{ }^{13} \mathrm{C}$ NMR for Azide A5
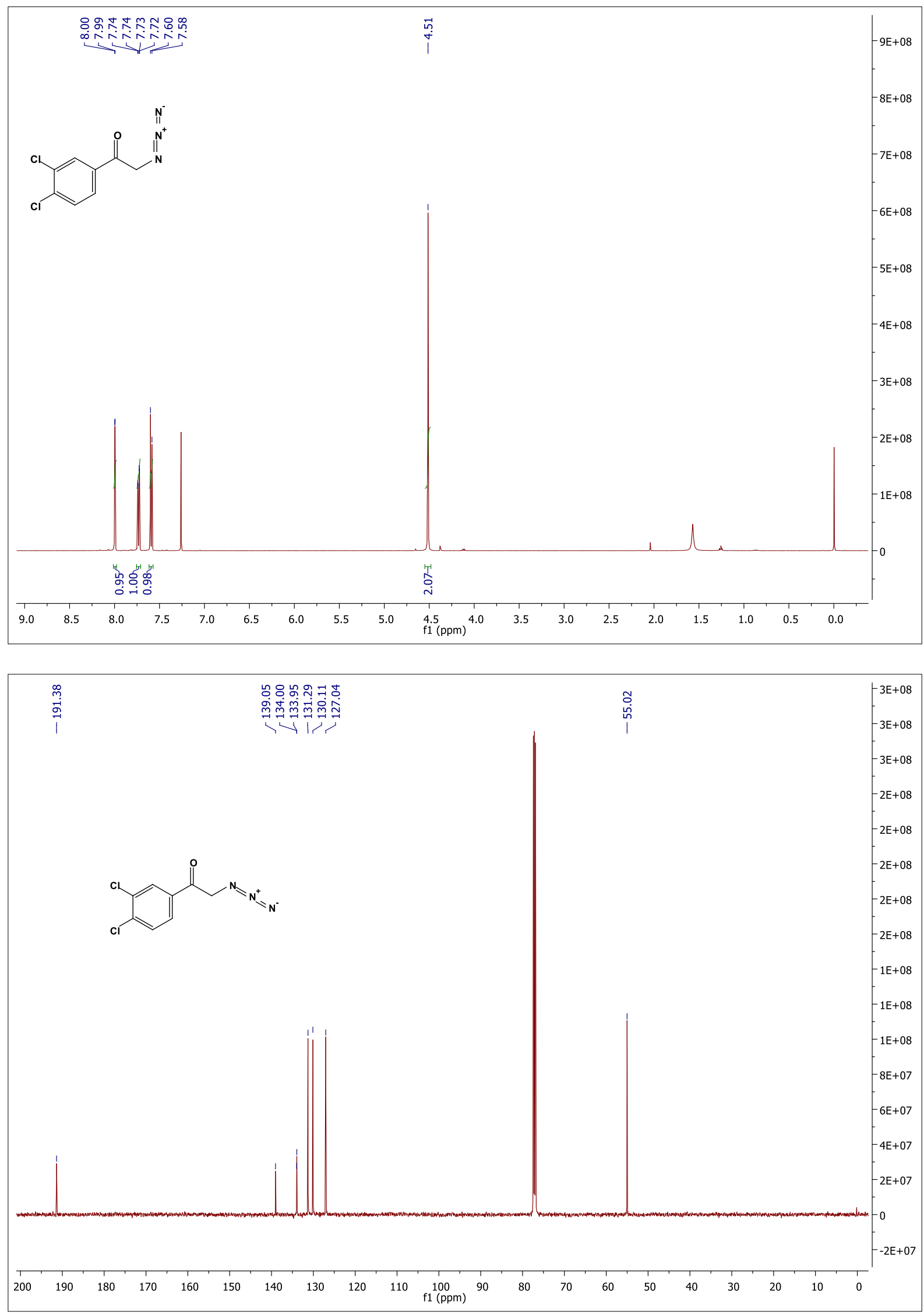
${ }^{1} \mathrm{H} \&{ }^{13} \mathrm{C}$ NMR for Azide A7
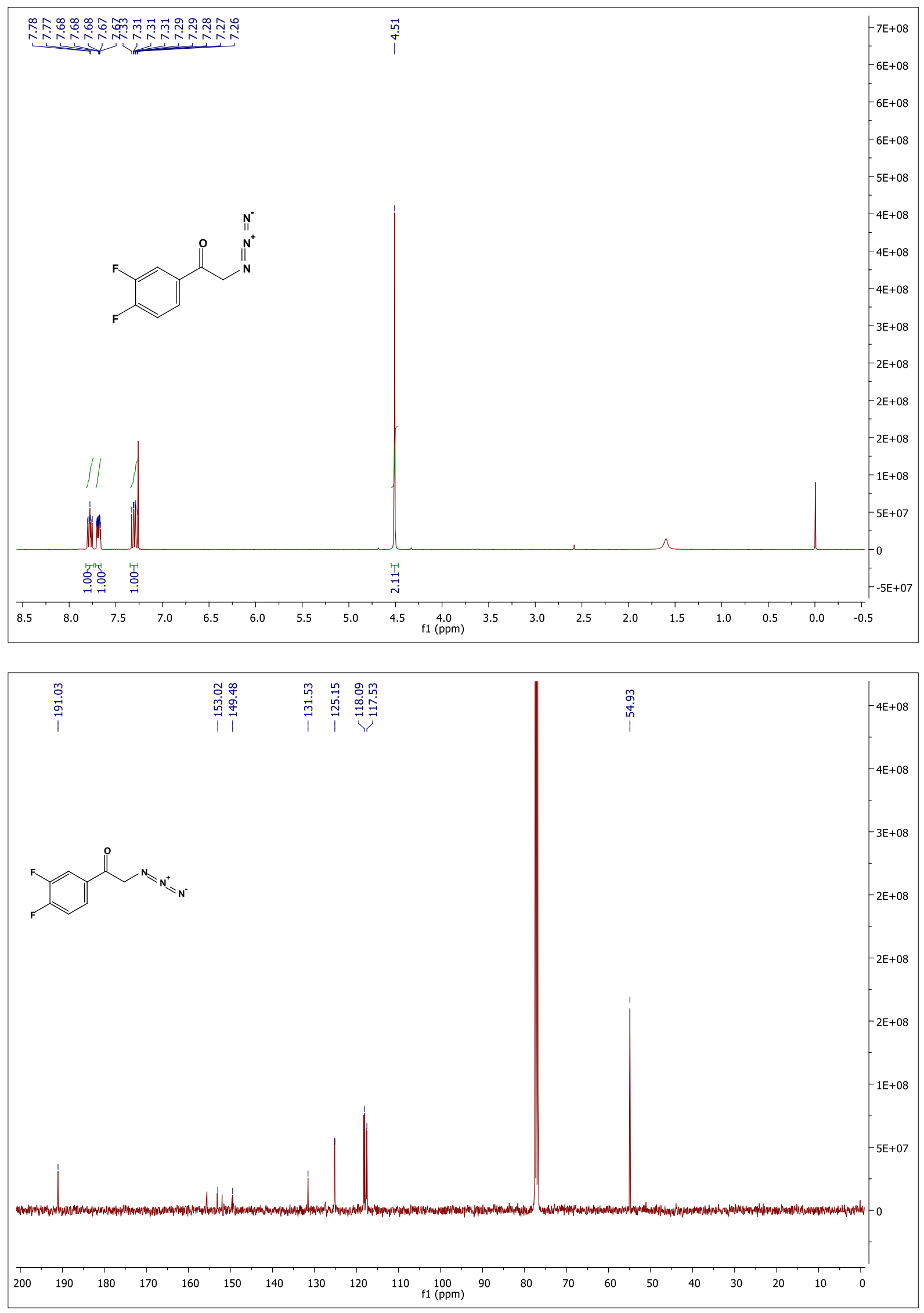
${ }^{1} \mathrm{H} \&{ }^{13} \mathrm{C}$ NMR for Azide A9
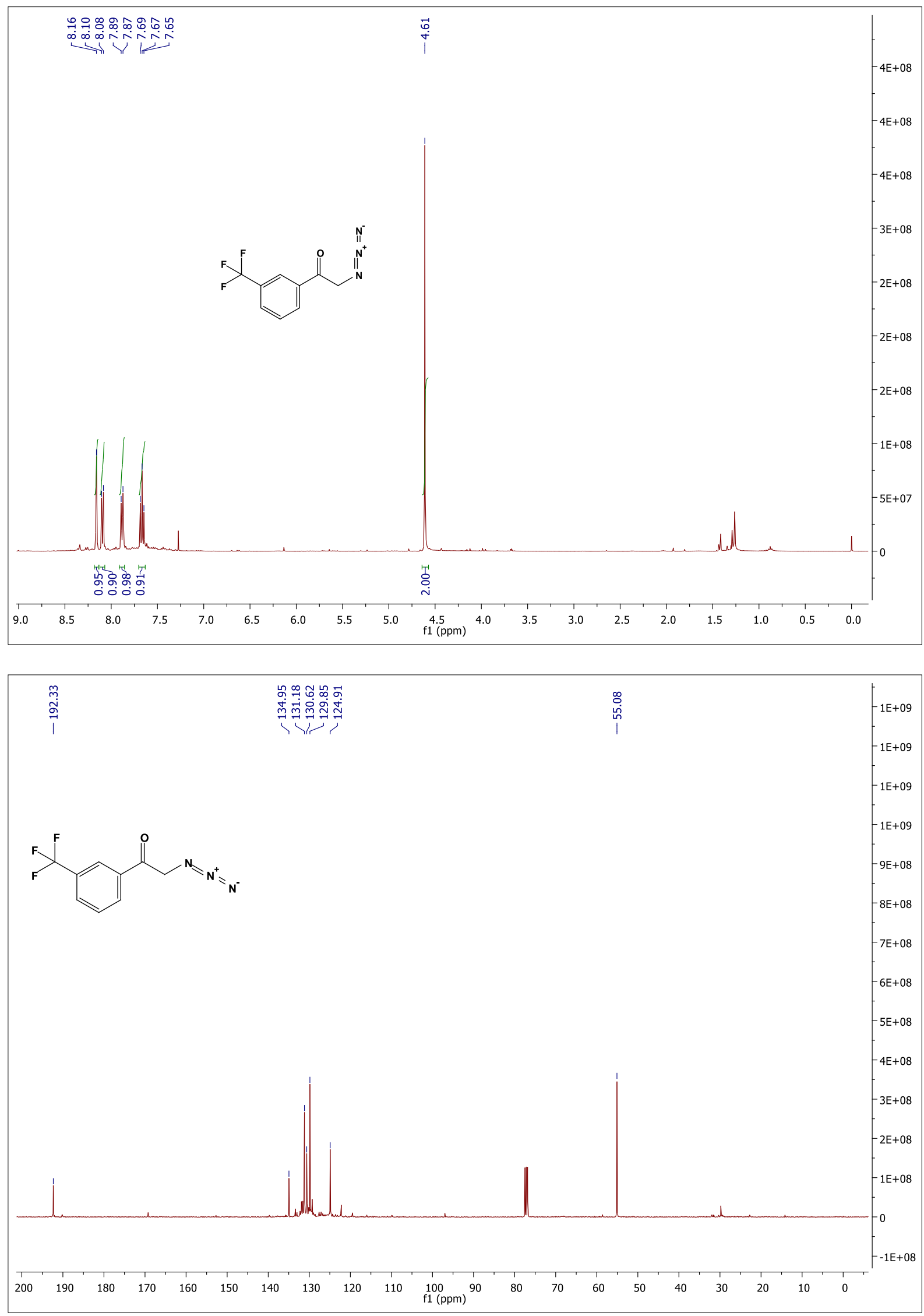
${ }^{1} \mathrm{H} \&{ }^{13} \mathrm{C}$ NMR for Azide A15
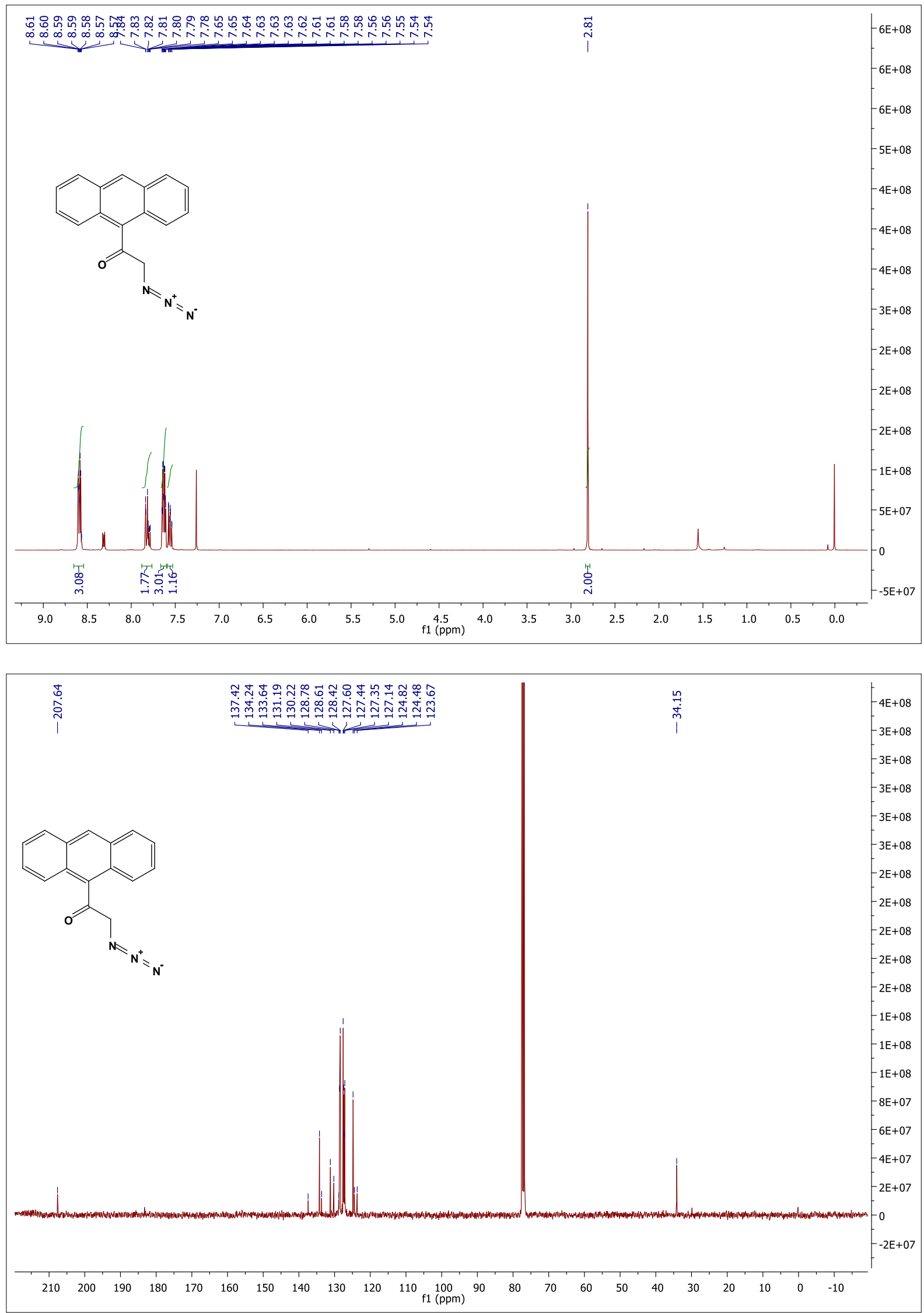
${ }^{1} \mathrm{H} \&{ }^{13} \mathrm{C}$ NMR for Azide A16
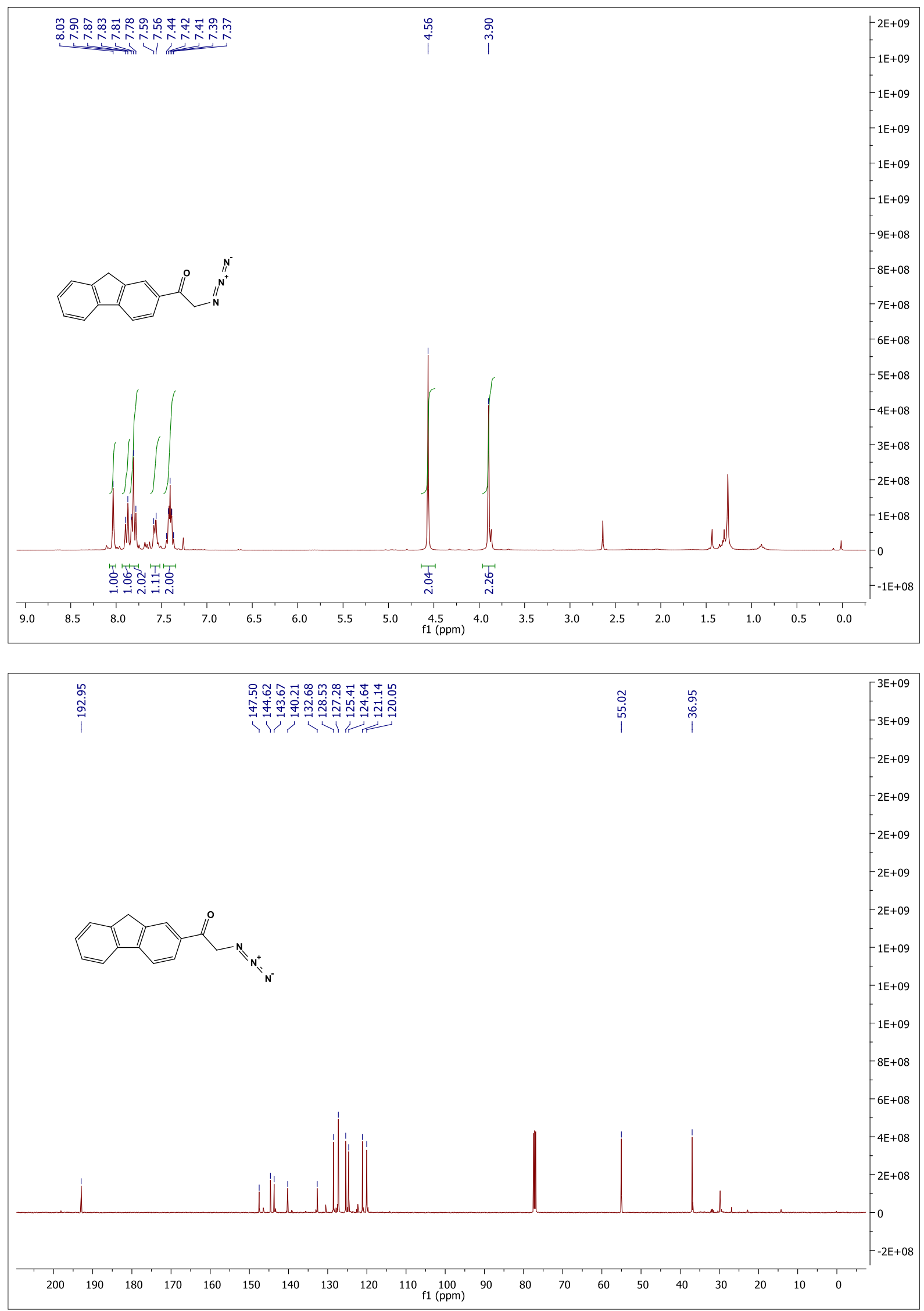
${ }^{1} \mathrm{H} \&{ }^{13} \mathrm{C}$ NMR for Azide A21
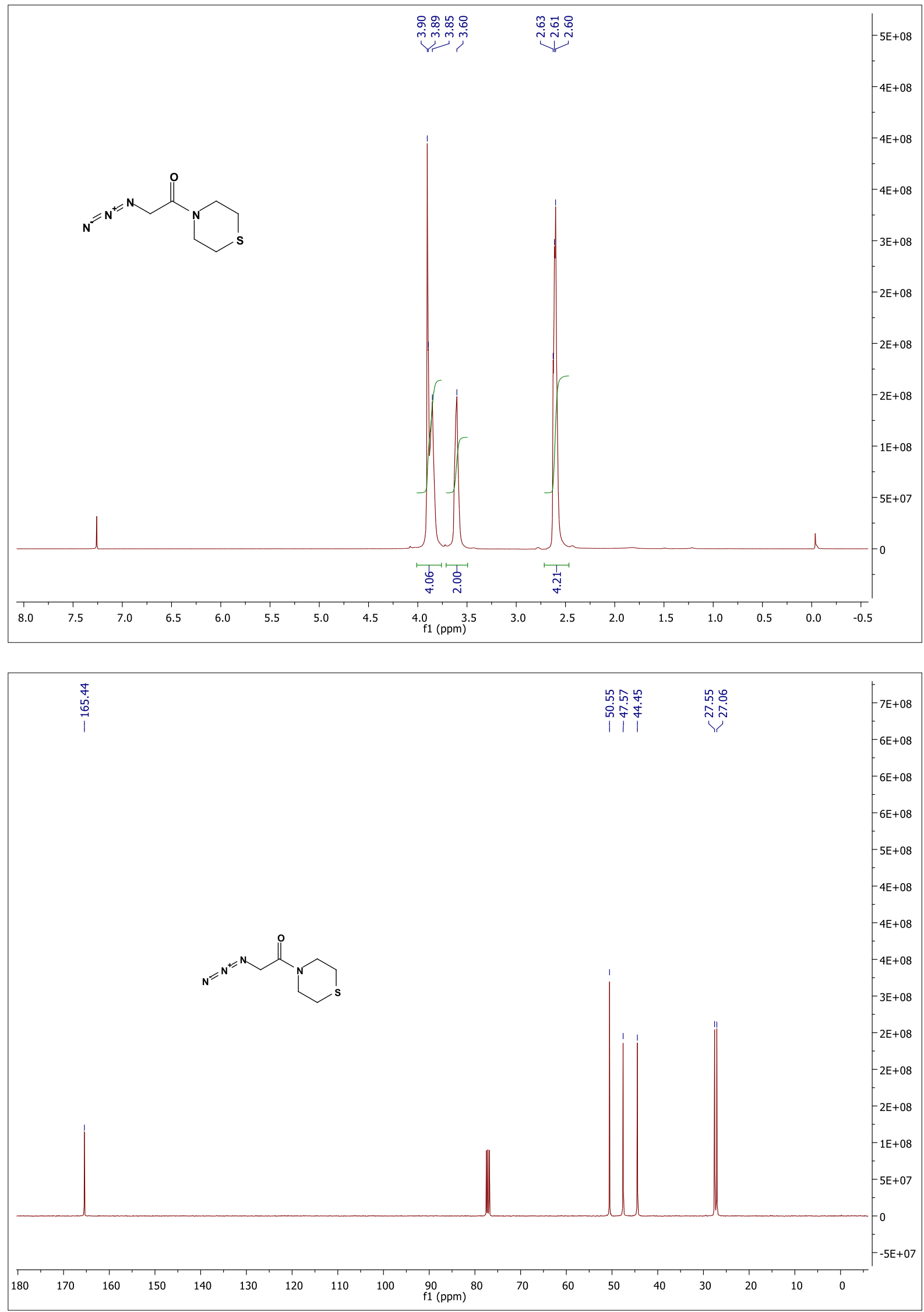
${ }^{1} \mathrm{H} \&{ }^{13} \mathrm{C}$ NMR for Azide A23
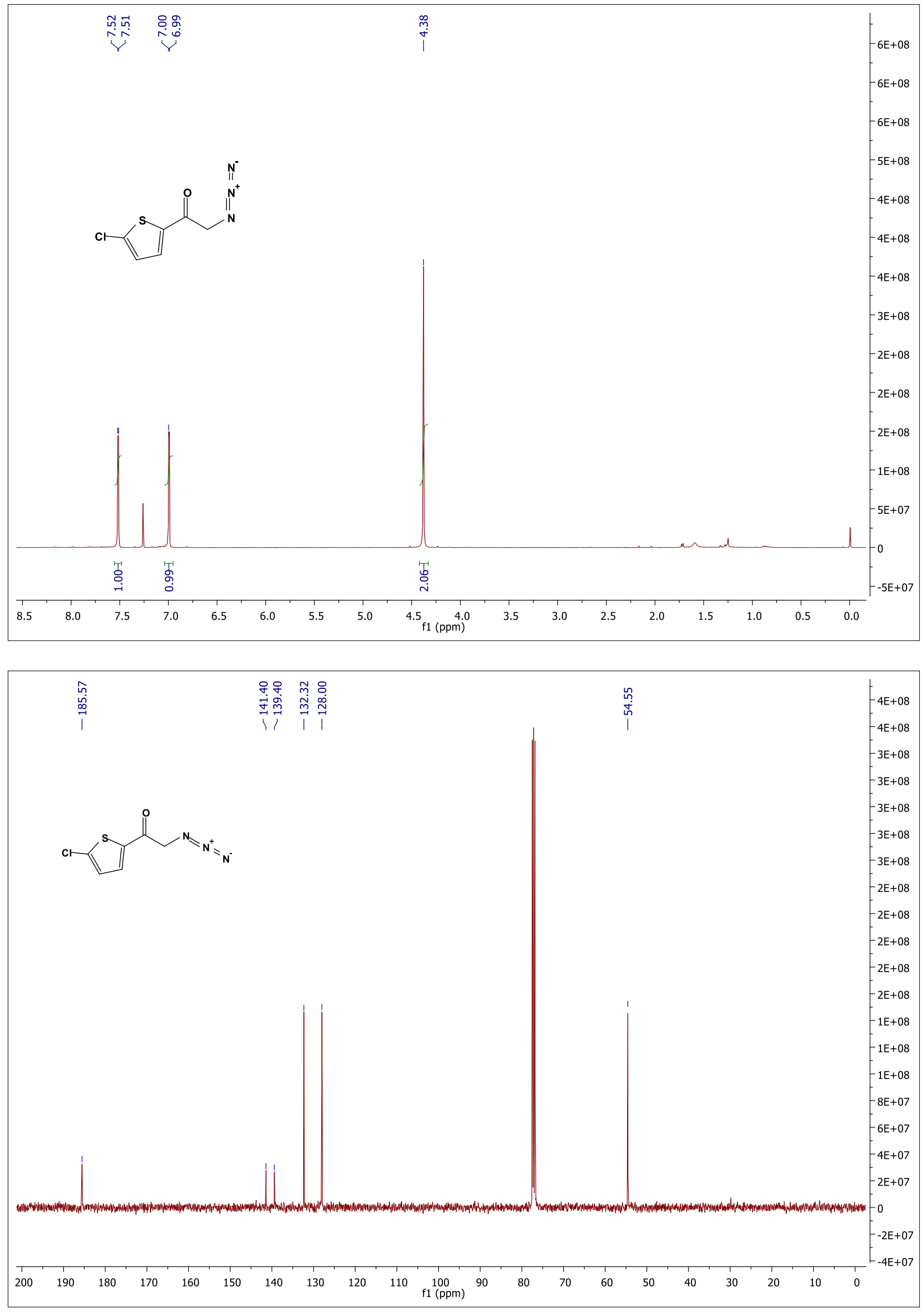
${ }^{1} \mathrm{H} \&{ }^{13} \mathrm{C}$ NMR for Azide A24
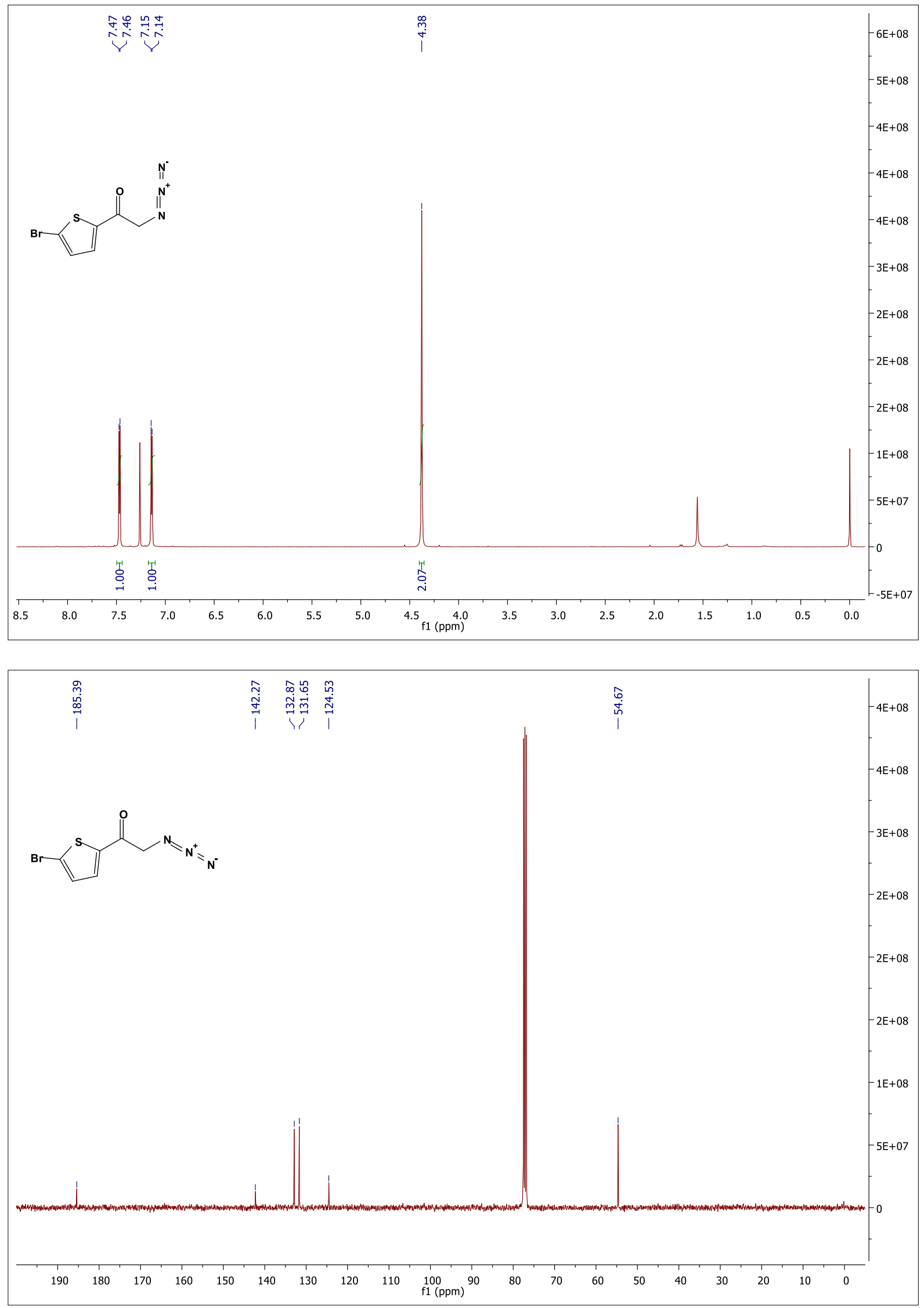
${ }^{1} \mathrm{H} \&{ }^{13} \mathrm{C}$ NMR for Azide A26
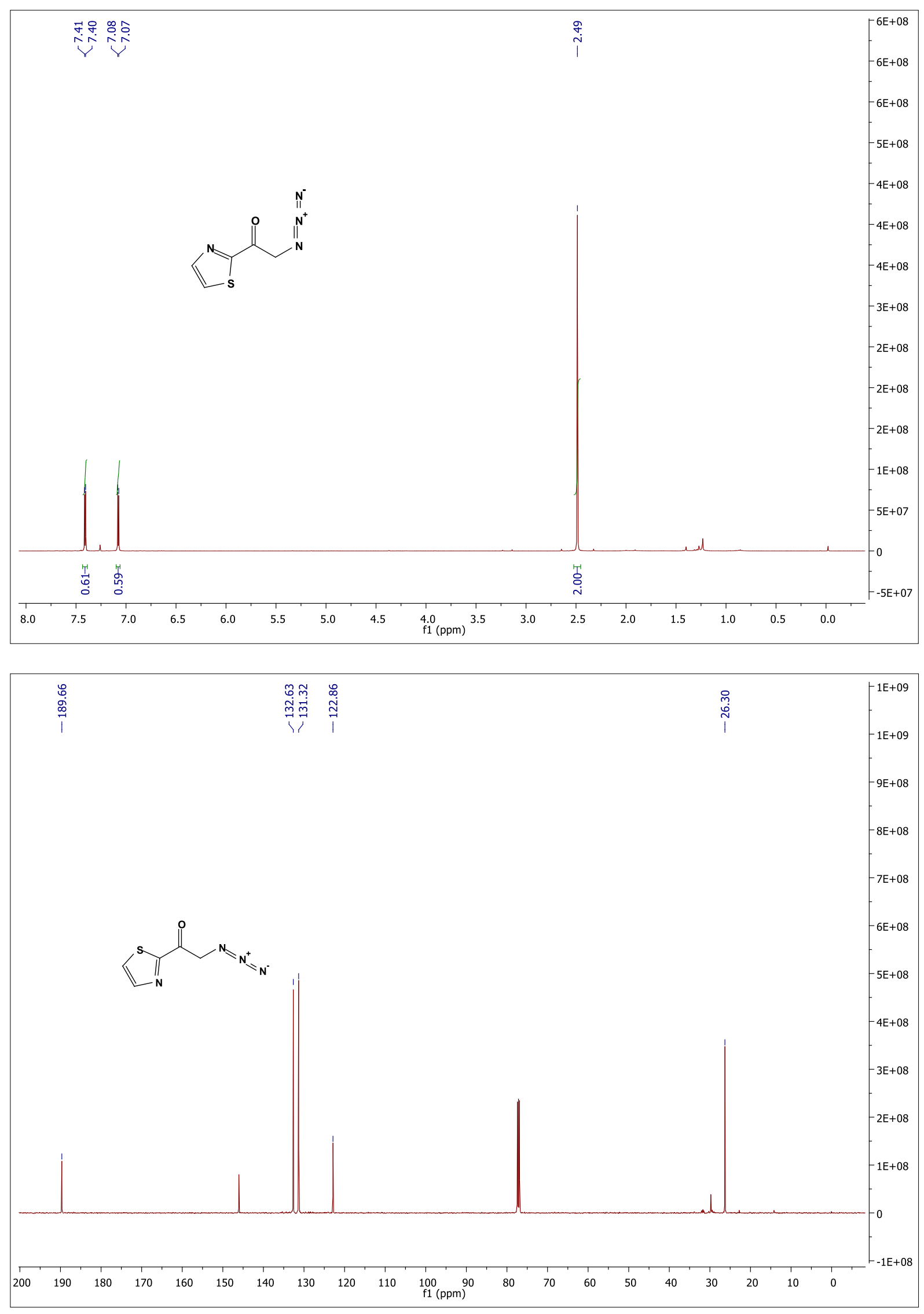

S50 
${ }^{1} \mathrm{H} \&{ }^{13} \mathrm{C}$ NMR for Azide A27
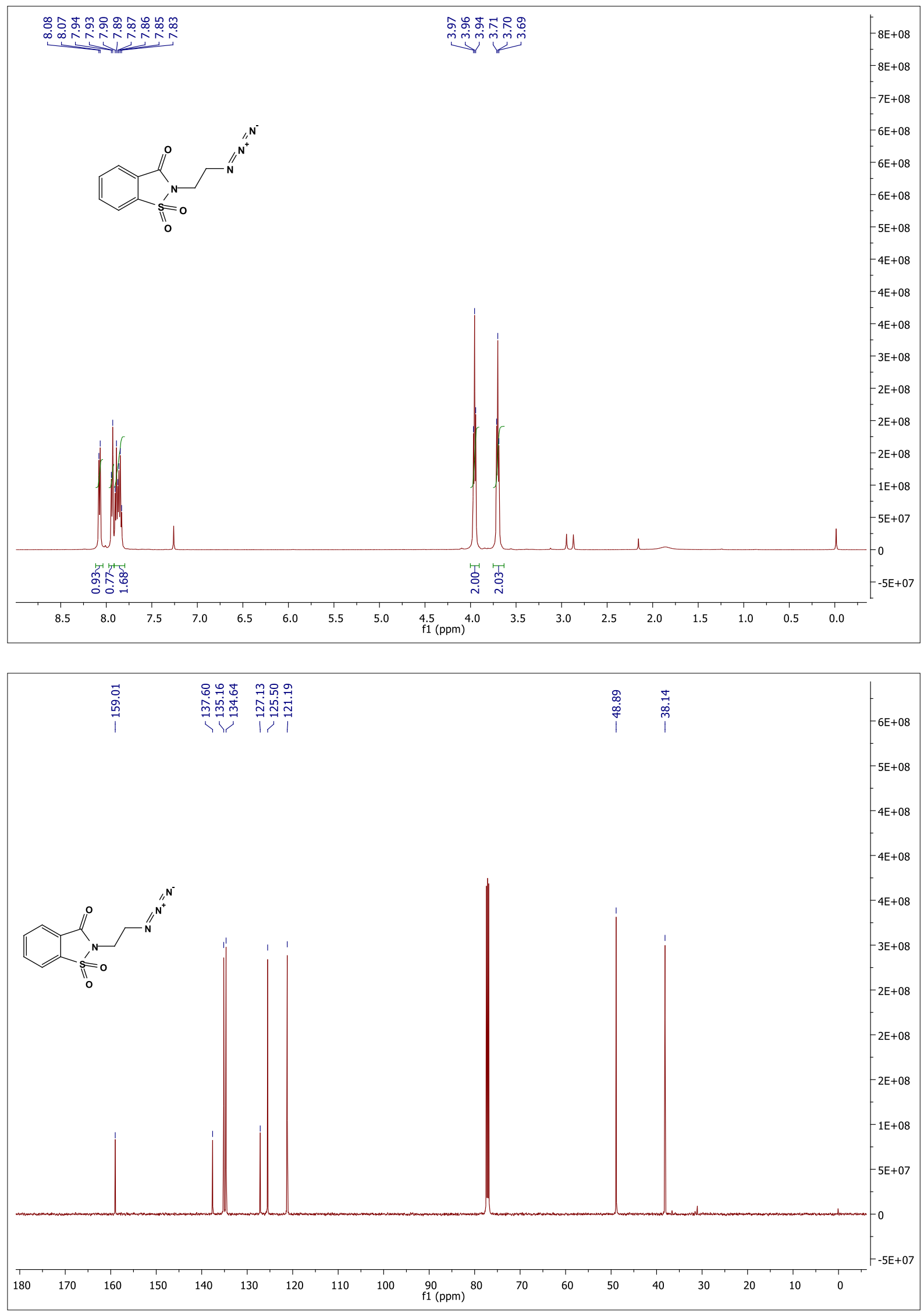
${ }^{1} \mathrm{H} \&{ }^{13} \mathrm{C}$ NMR for Azide A28
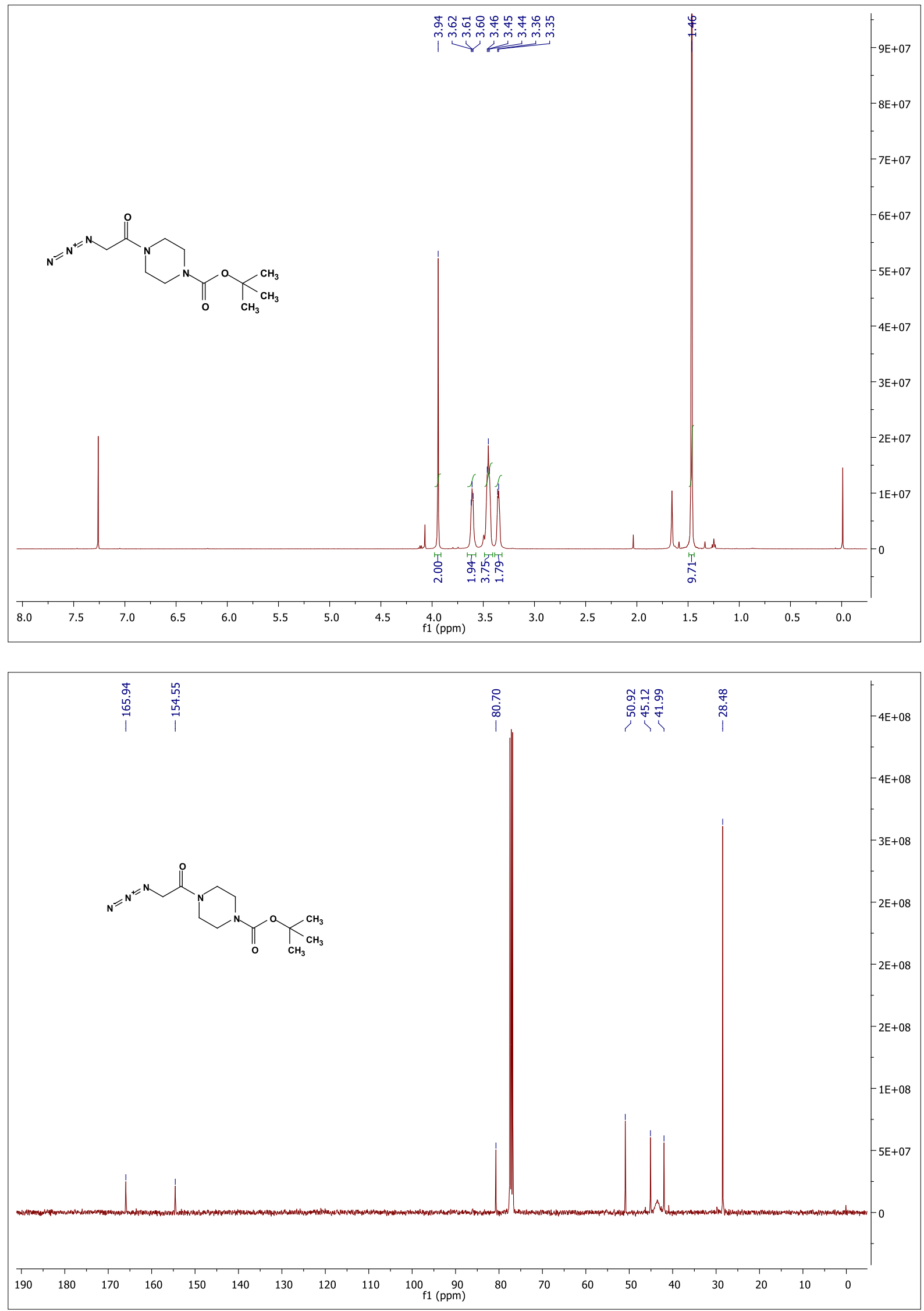
${ }^{1} \mathrm{H} \&{ }^{13} \mathrm{C}$ NMR for Azide A29
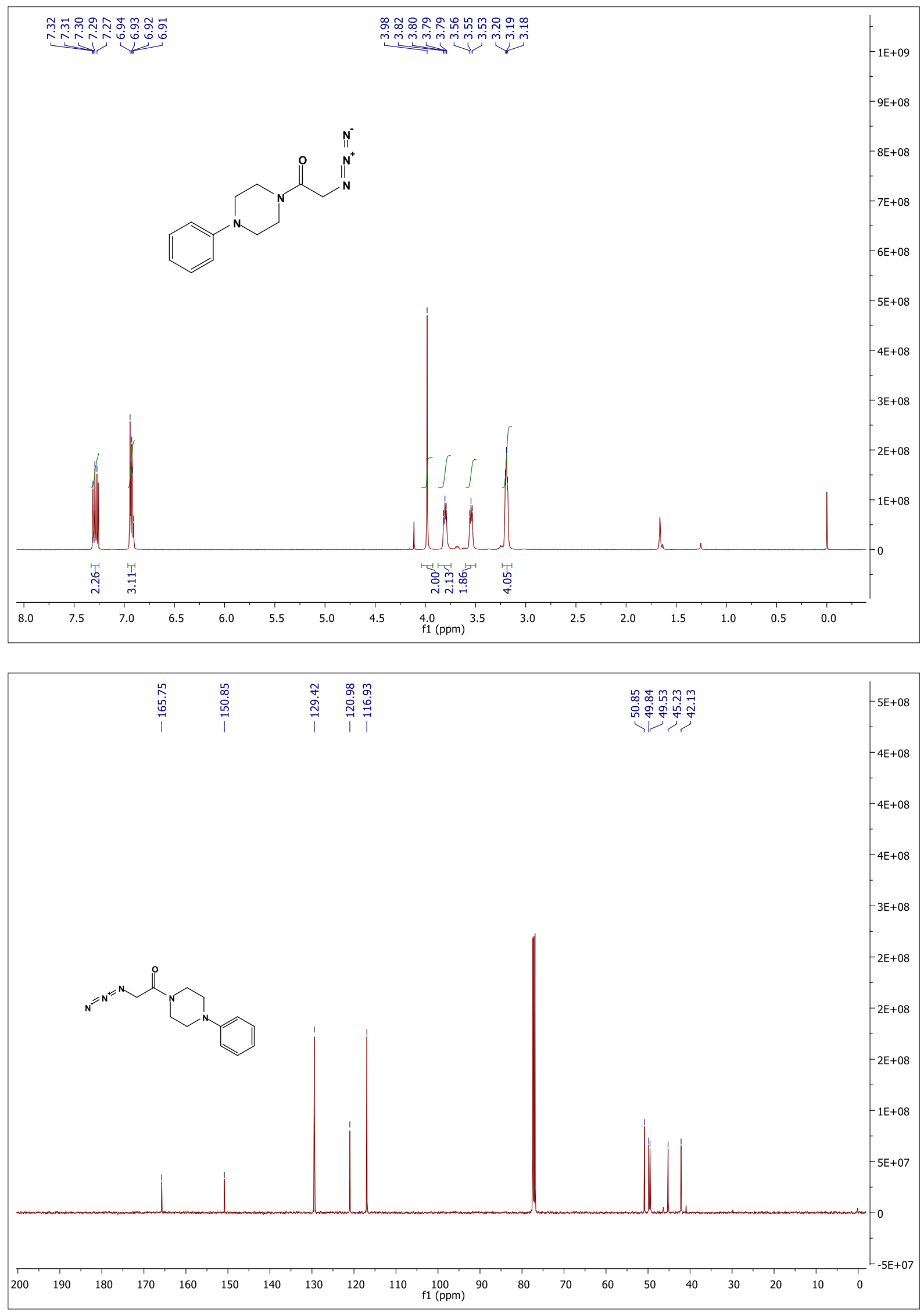
${ }^{1} \mathrm{H} \&^{13} \mathrm{C}$ NMR for Azide A30
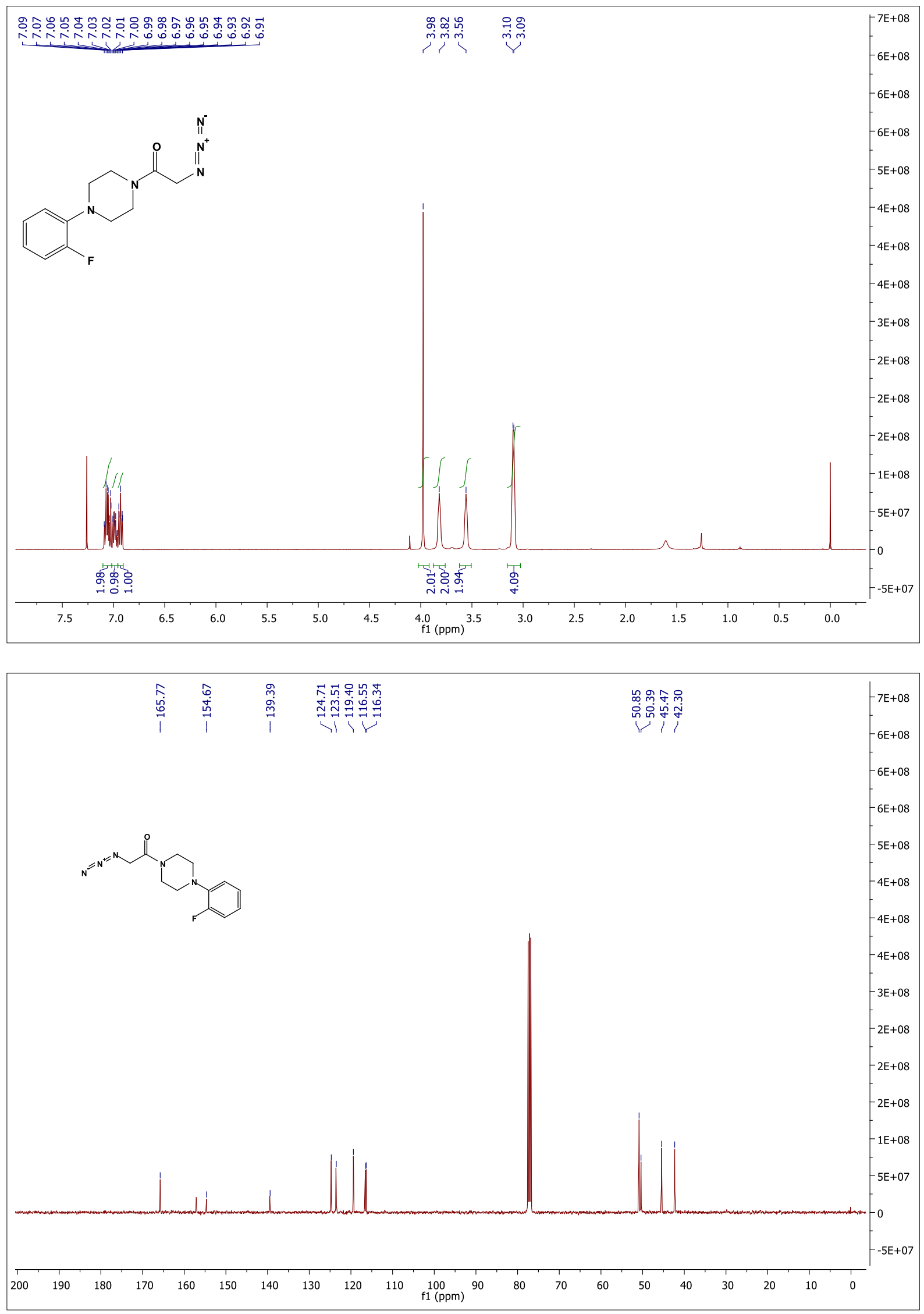
${ }^{1} \mathrm{H} \&^{13} \mathrm{C}$ NMR for Azide A31
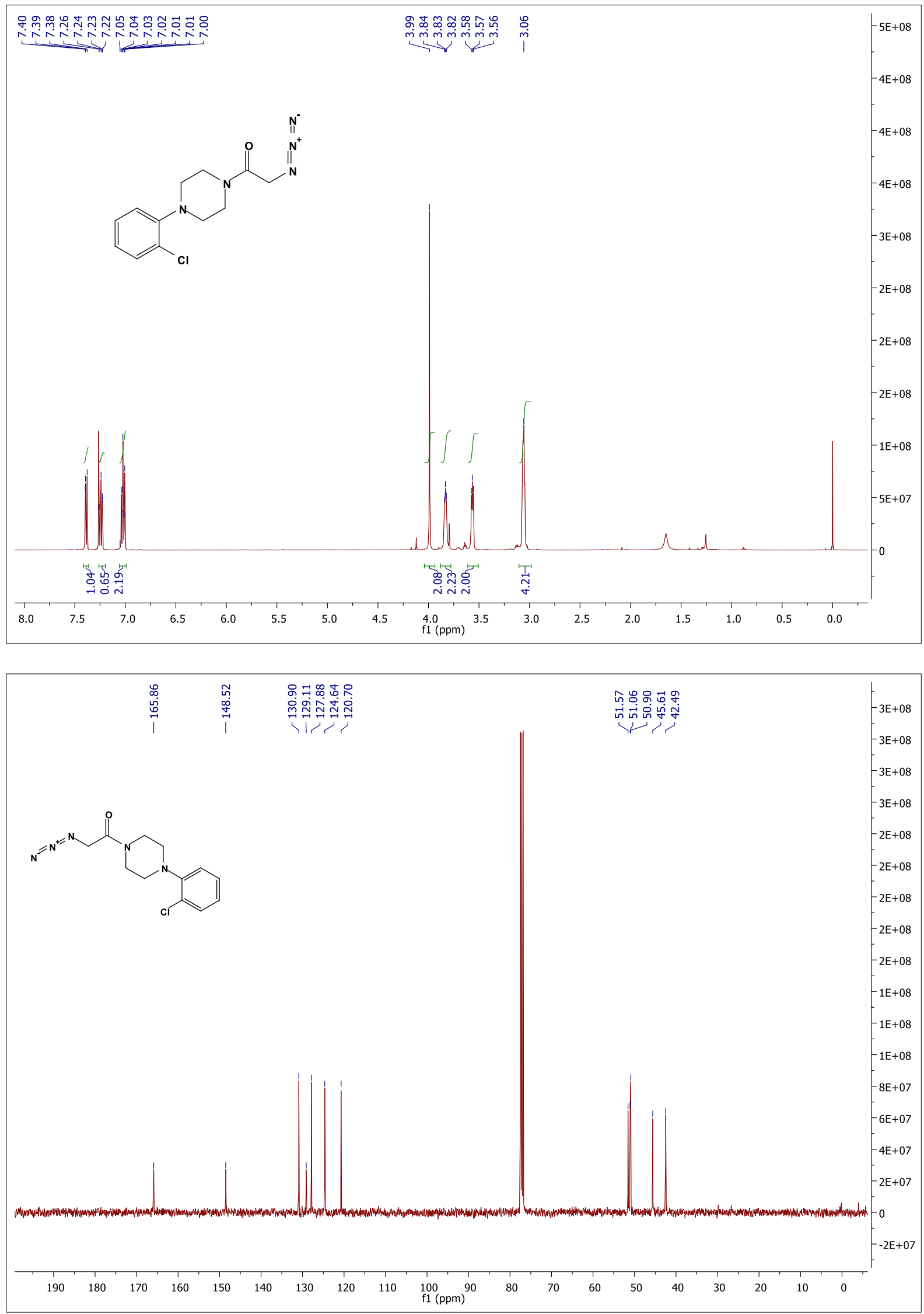
${ }^{1} \mathrm{H} \&^{13} \mathrm{C}$ NMR for Azide A32
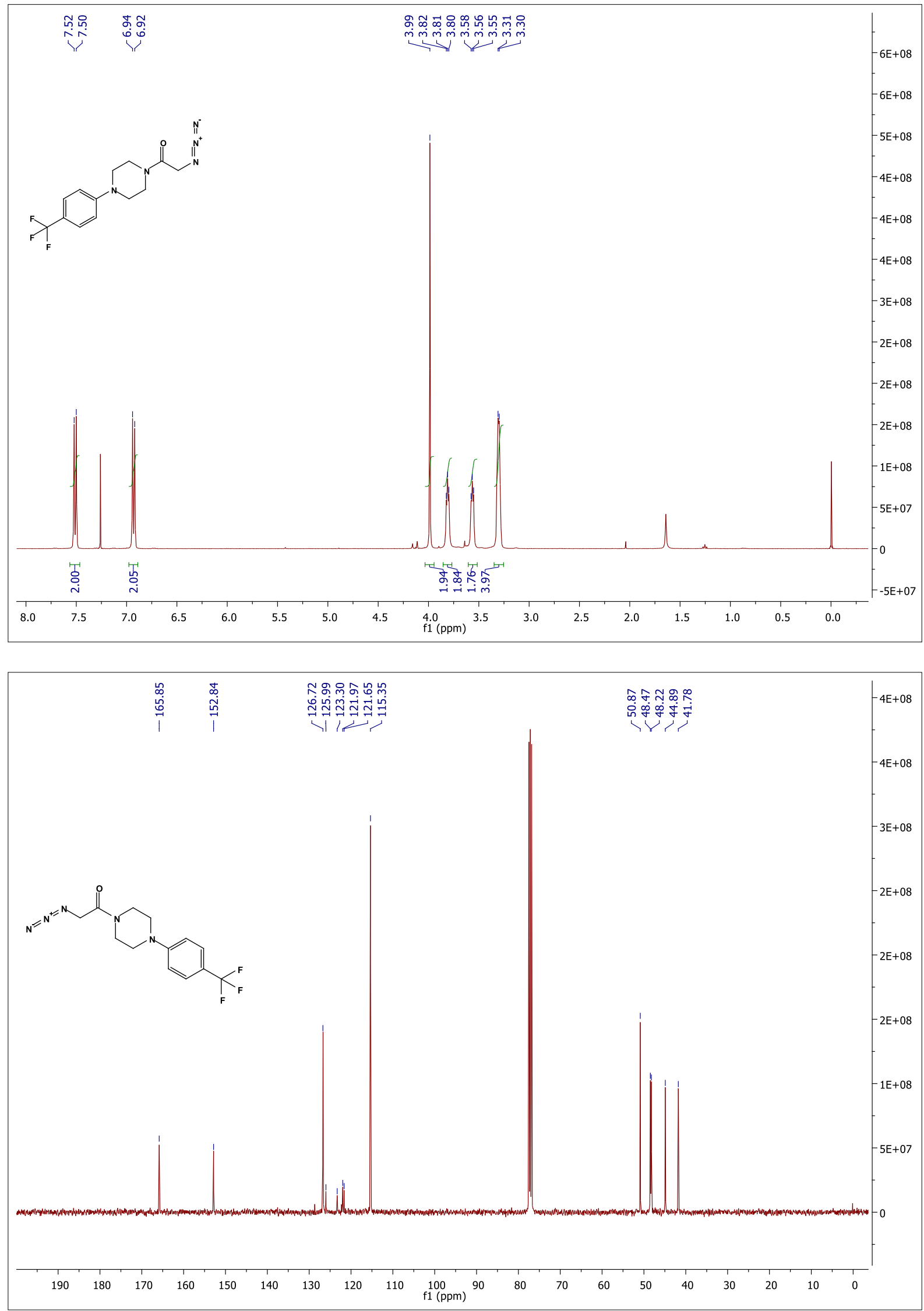
${ }^{1} \mathrm{H} \&{ }^{13} \mathrm{C}$ NMR for Azide A33
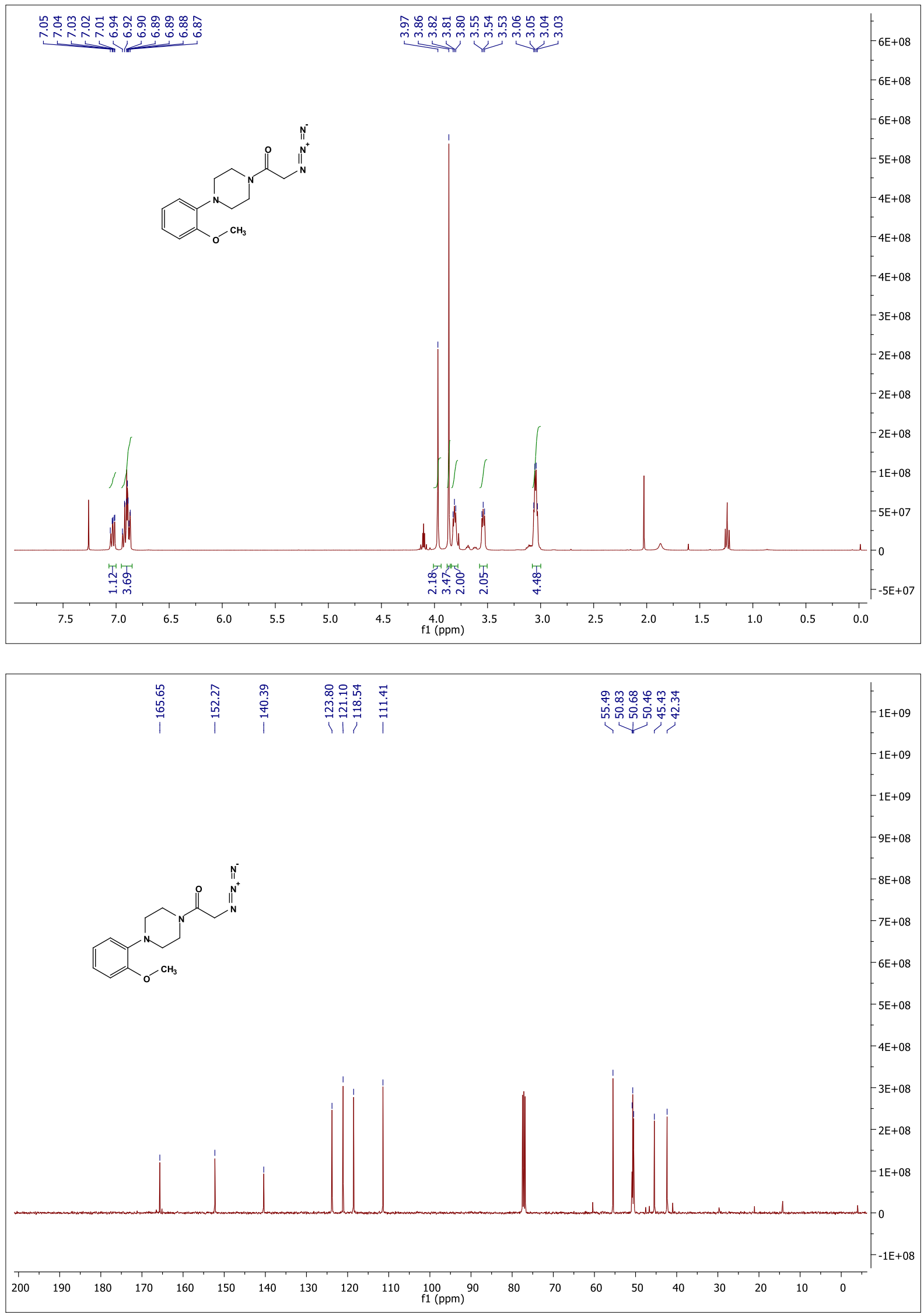
${ }^{1} \mathrm{H} \&{ }^{13} \mathrm{C}$ NMR for Azide A34
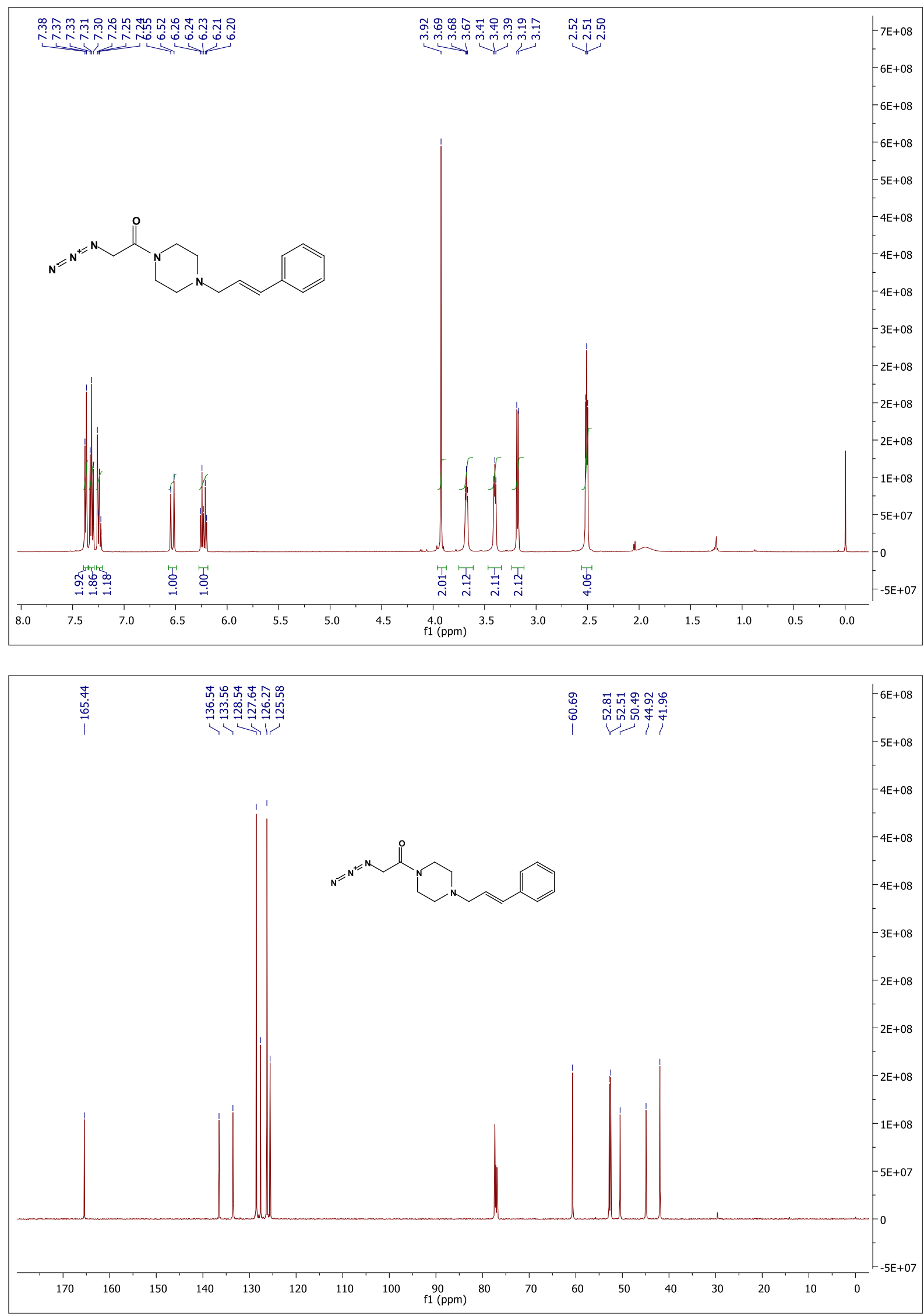
${ }^{1} \mathrm{H} \&{ }^{13} \mathrm{C}$ NMR for Azide A35
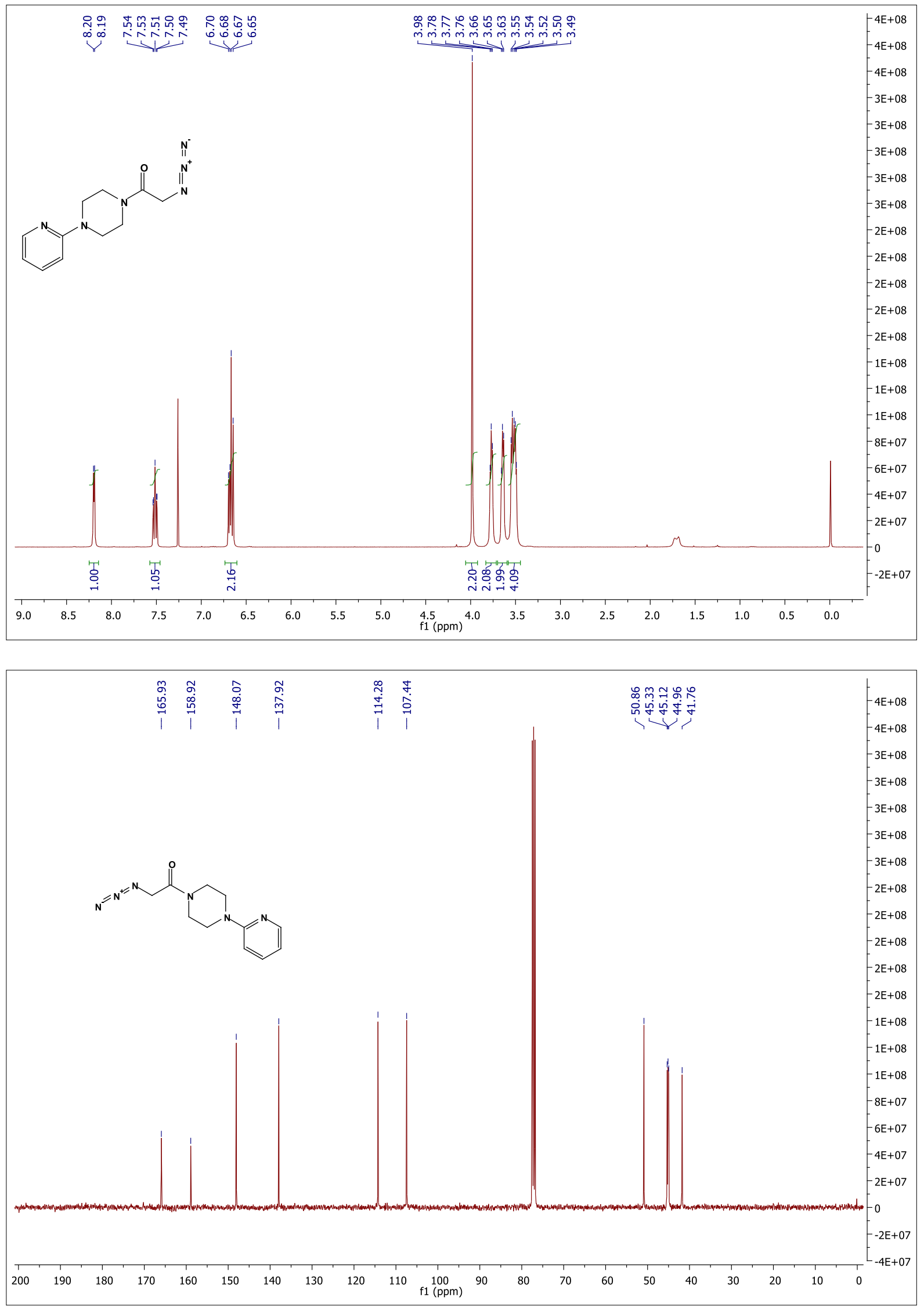
${ }^{1} \mathrm{H} \&{ }^{13} \mathrm{C}$ NMR for compound 8
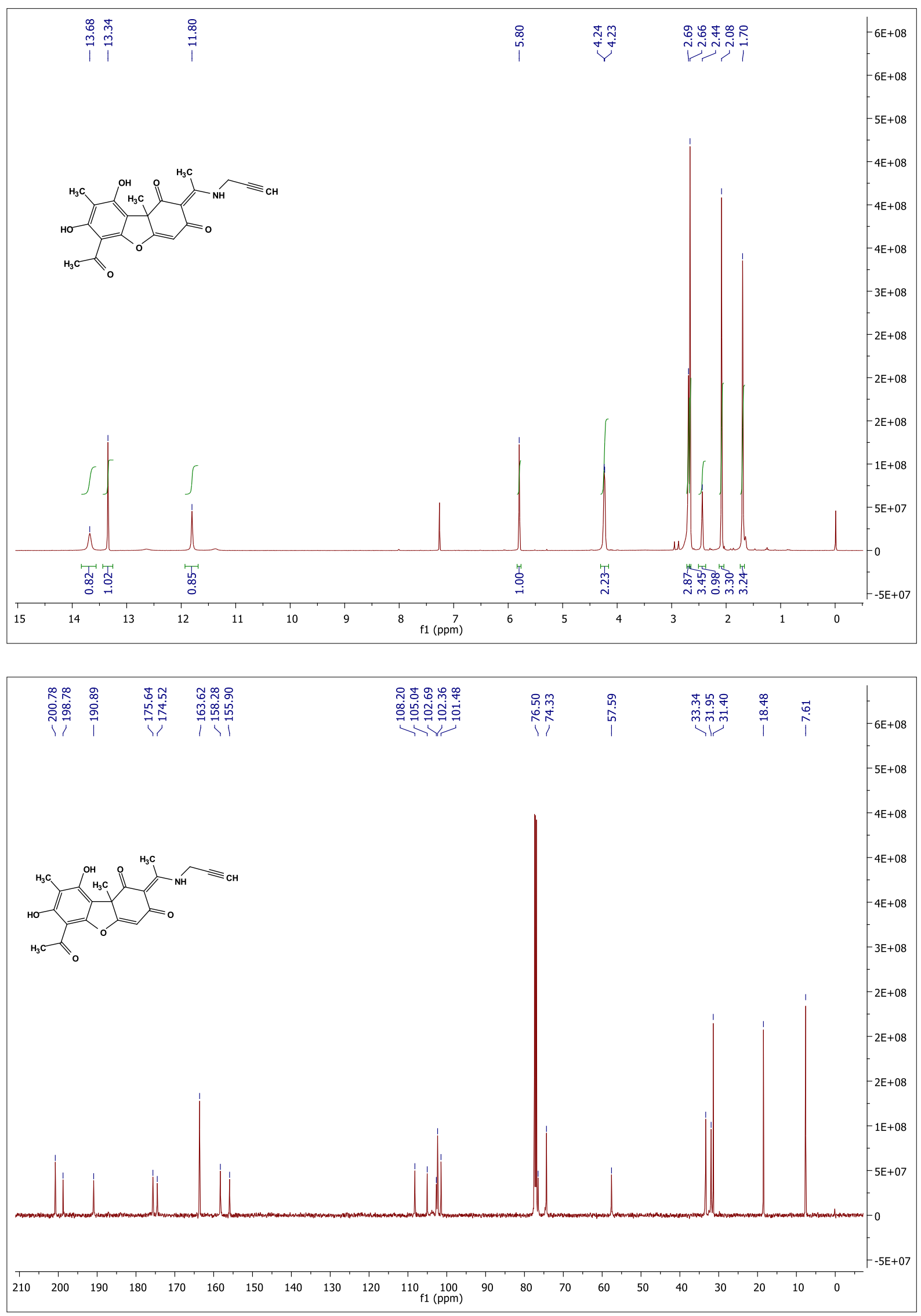
${ }^{1} \mathrm{H} \&{ }^{13} \mathrm{C}$ NMR for compound 10
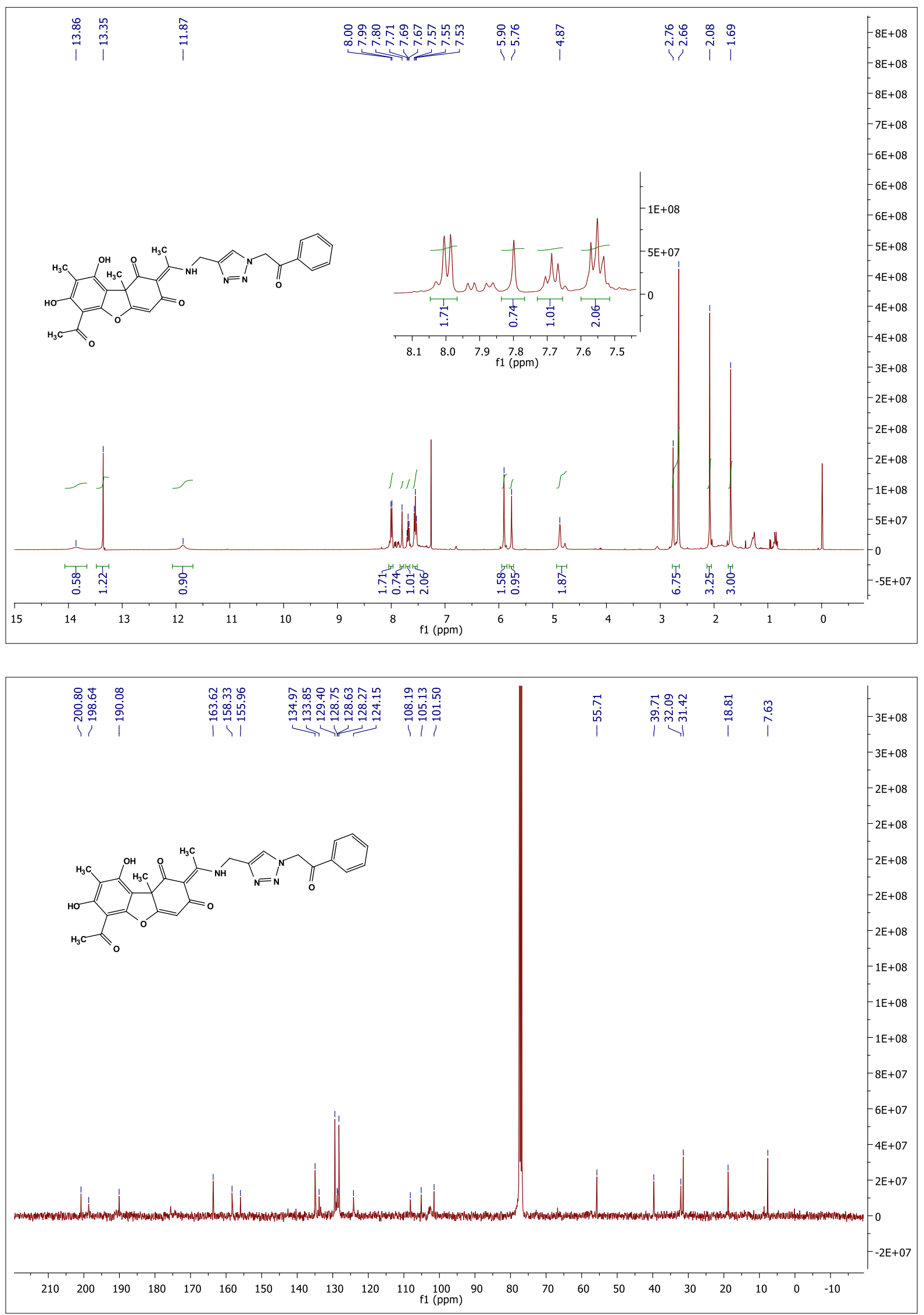
${ }^{1} \mathrm{H} \&{ }^{13} \mathrm{C}$ NMR for compound 11
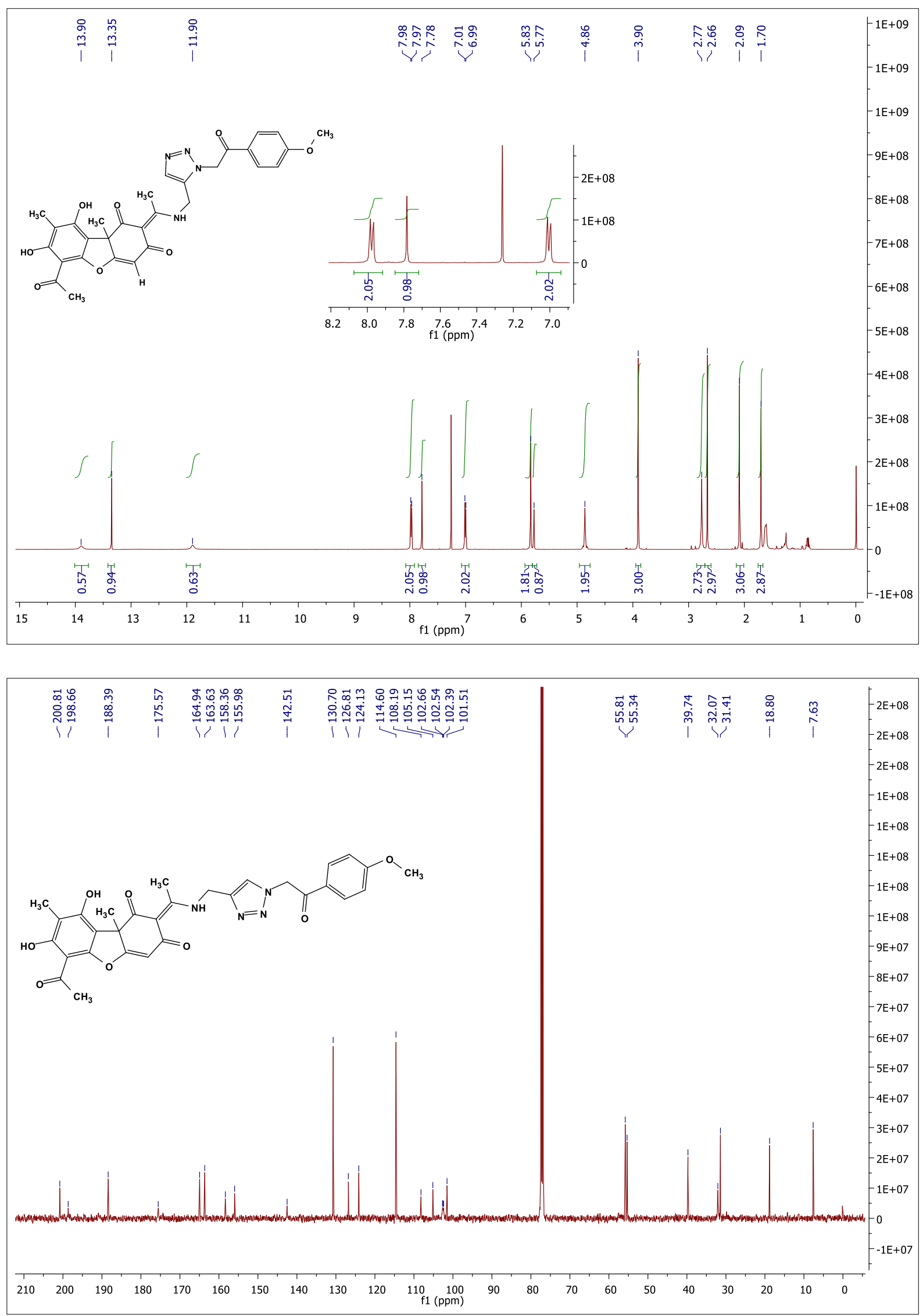
${ }^{1} \mathrm{H} \&{ }^{13} \mathrm{C}$ NMR for compound 12
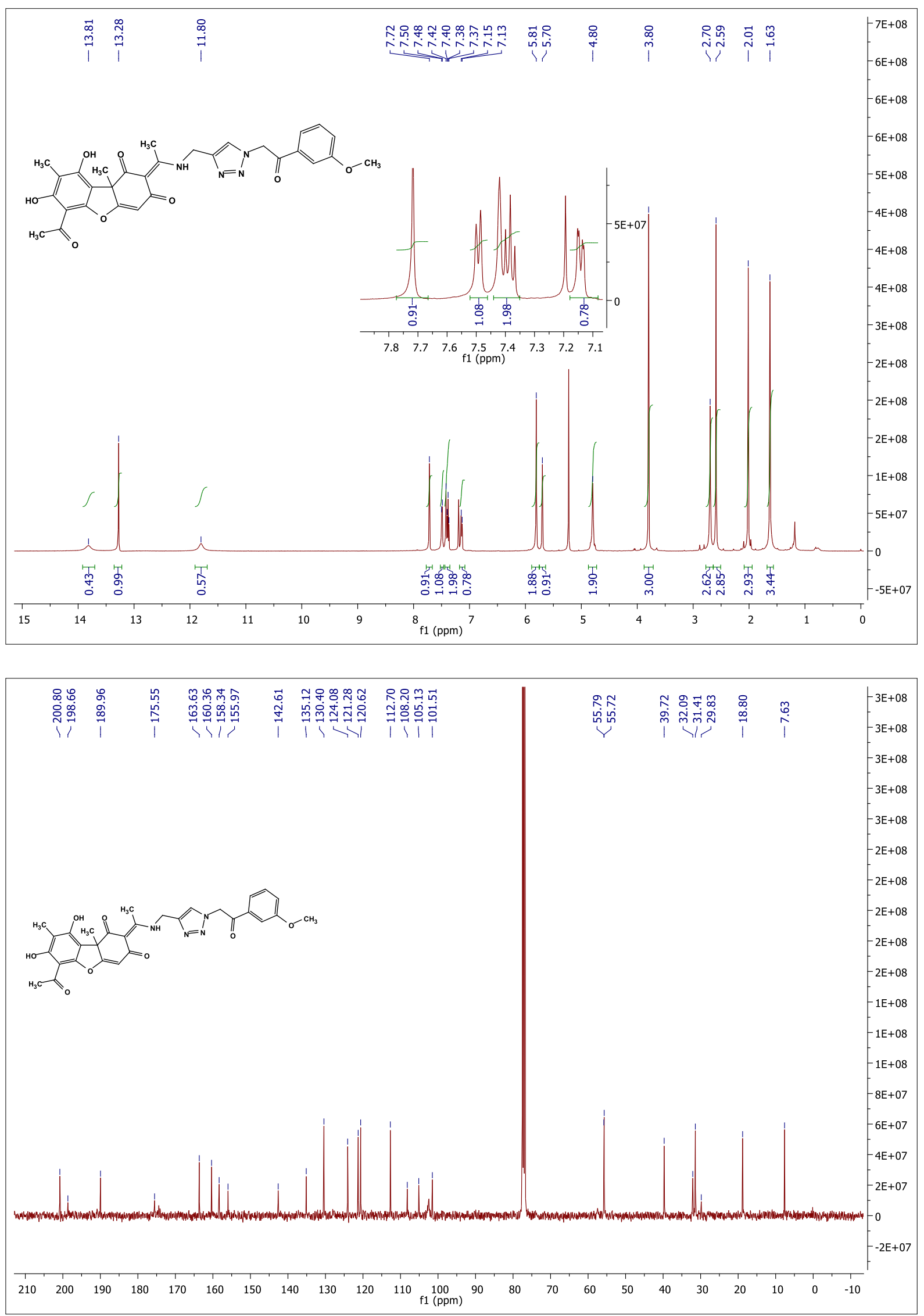
${ }^{1} \mathrm{H} \&{ }^{13} \mathrm{C}$ NMR for compound 13
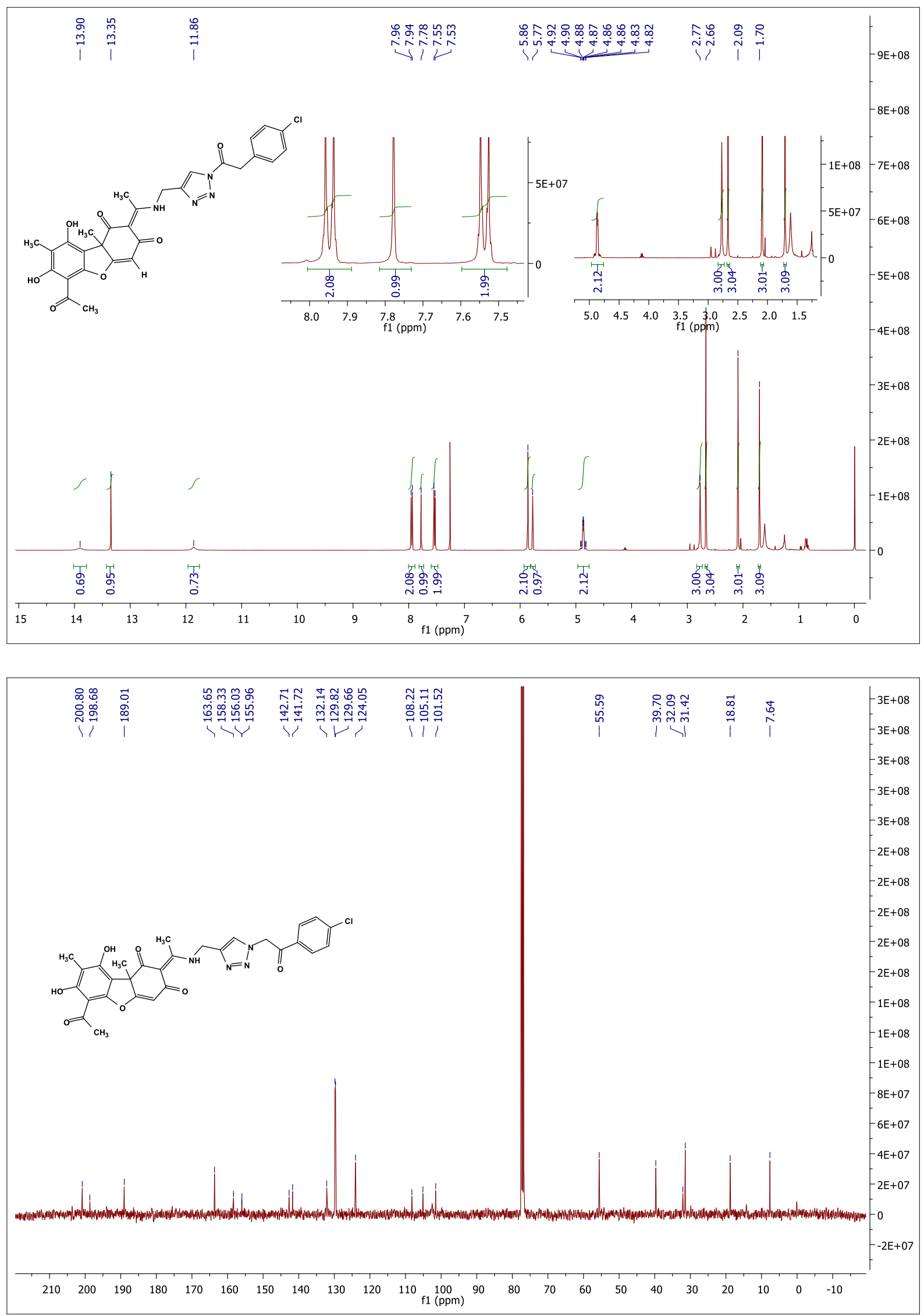
${ }^{1} \mathrm{H} \&{ }^{13} \mathrm{C}$ NMR for compound 14
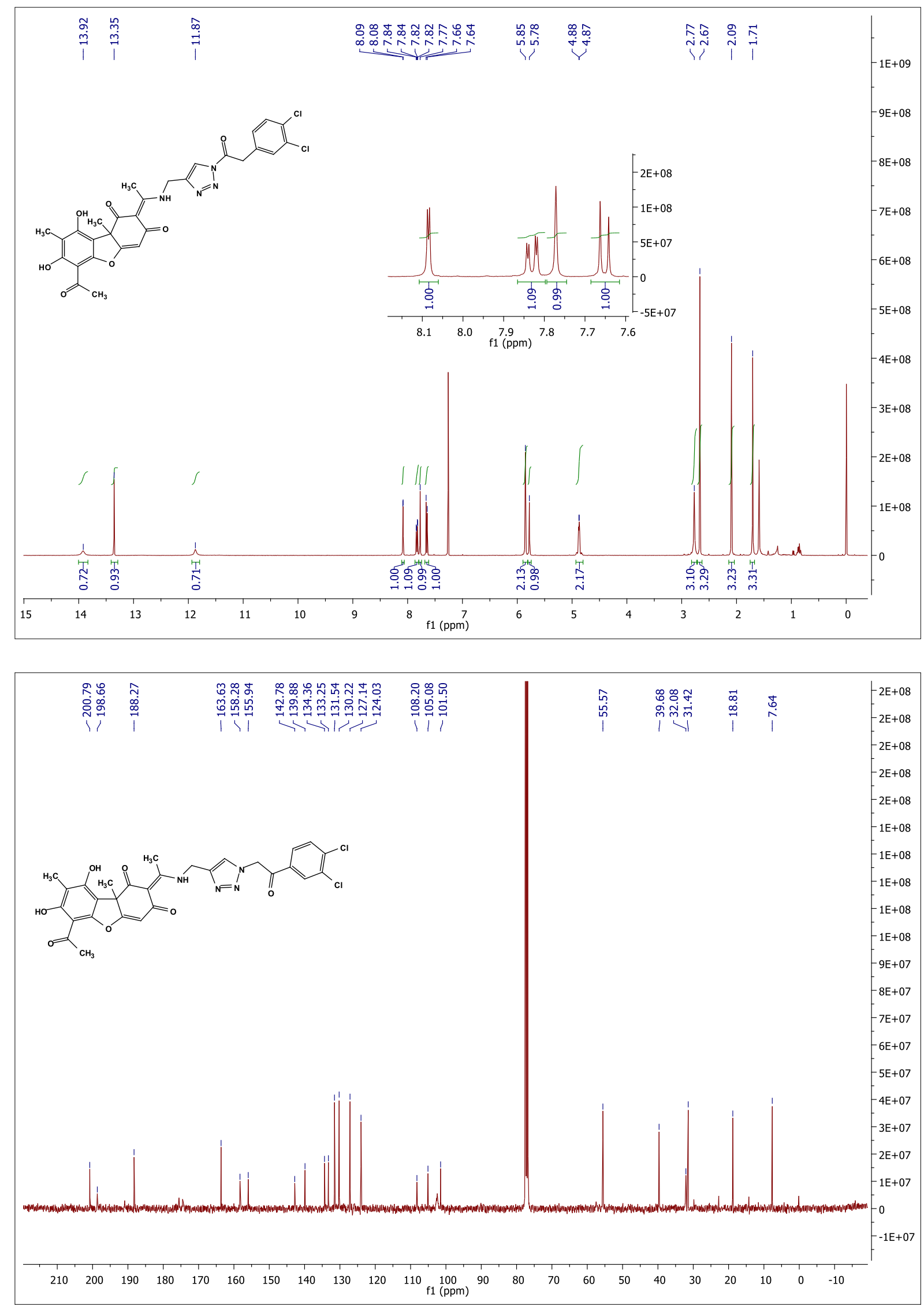
${ }^{1} \mathrm{H} \&{ }^{13} \mathrm{C}$ NMR for compound 15
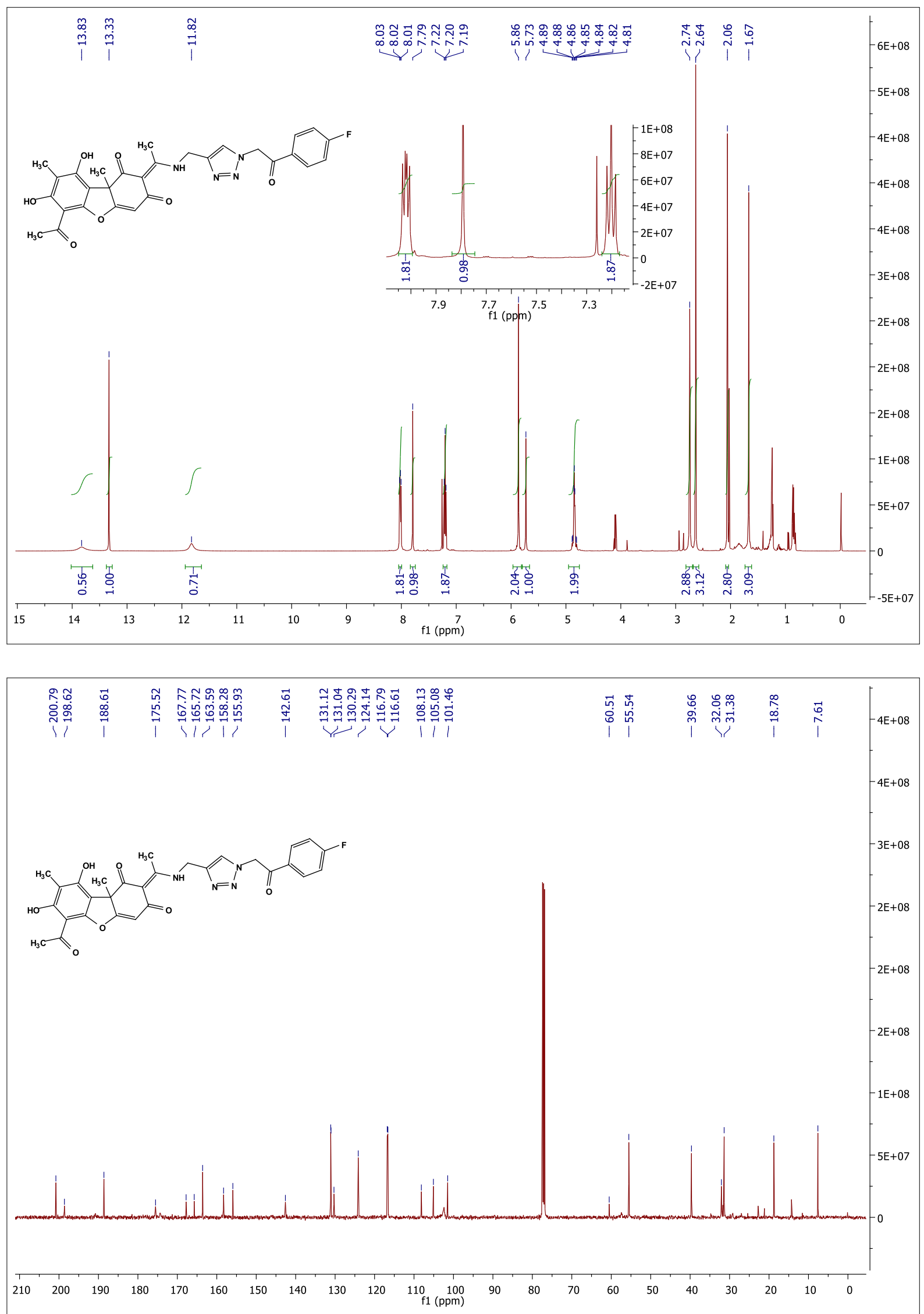
${ }^{1} \mathrm{H} \&{ }^{13} \mathrm{C}$ NMR for compound 16
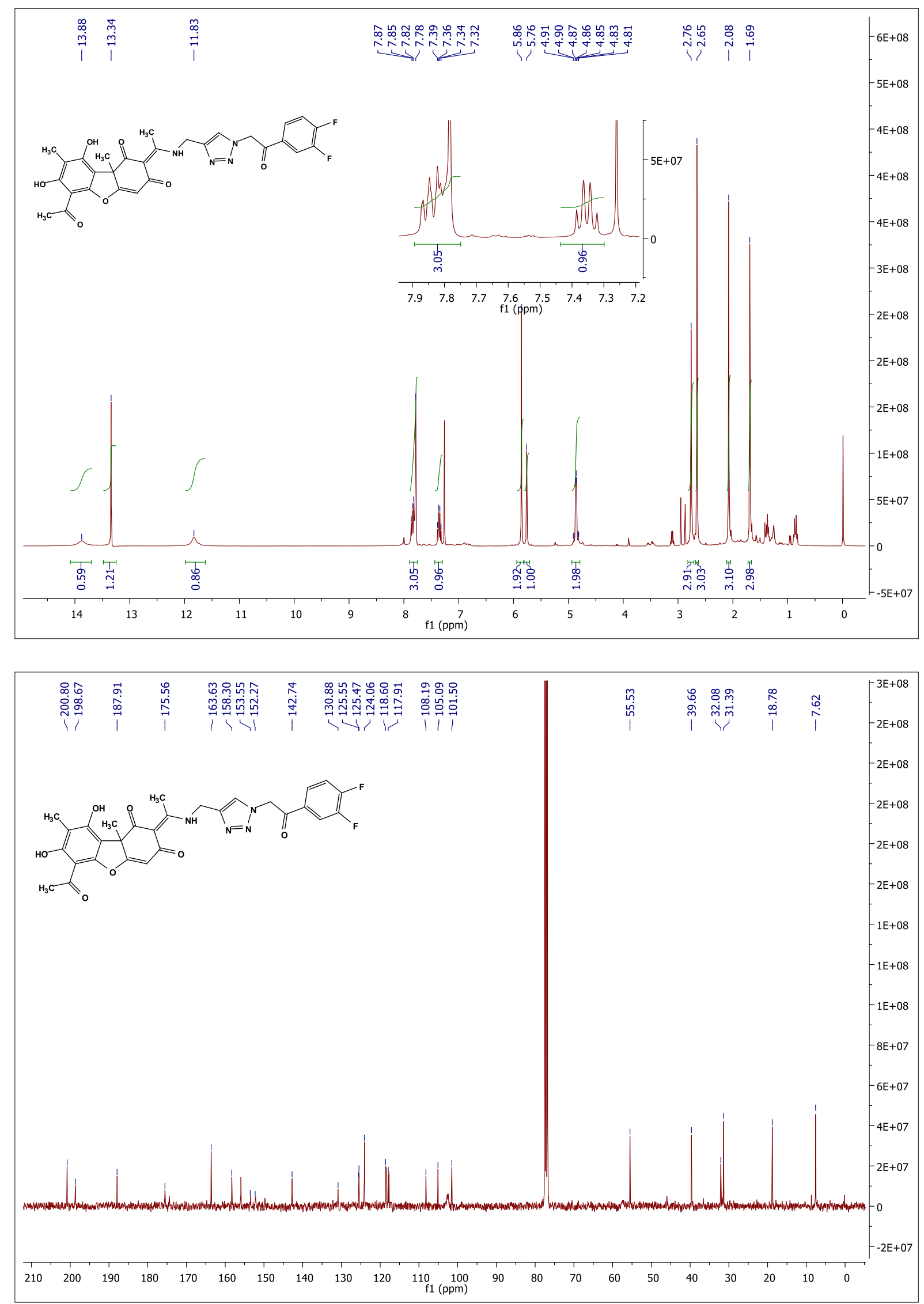
${ }^{1} \mathrm{H} \&{ }^{13} \mathrm{C}$ NMR for compound 17
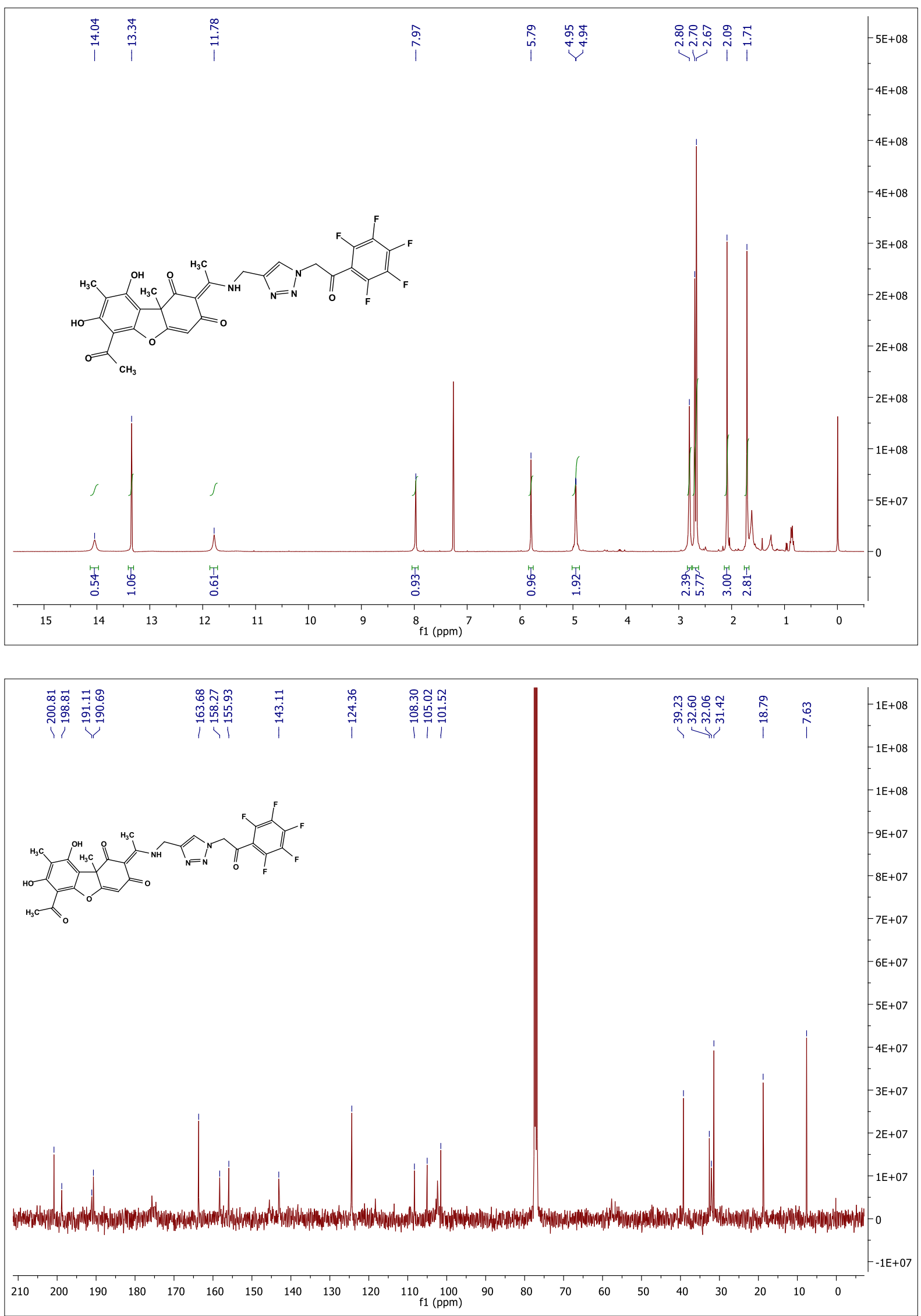
${ }^{1} \mathrm{H} \&{ }^{13} \mathrm{C}$ NMR for compound 18
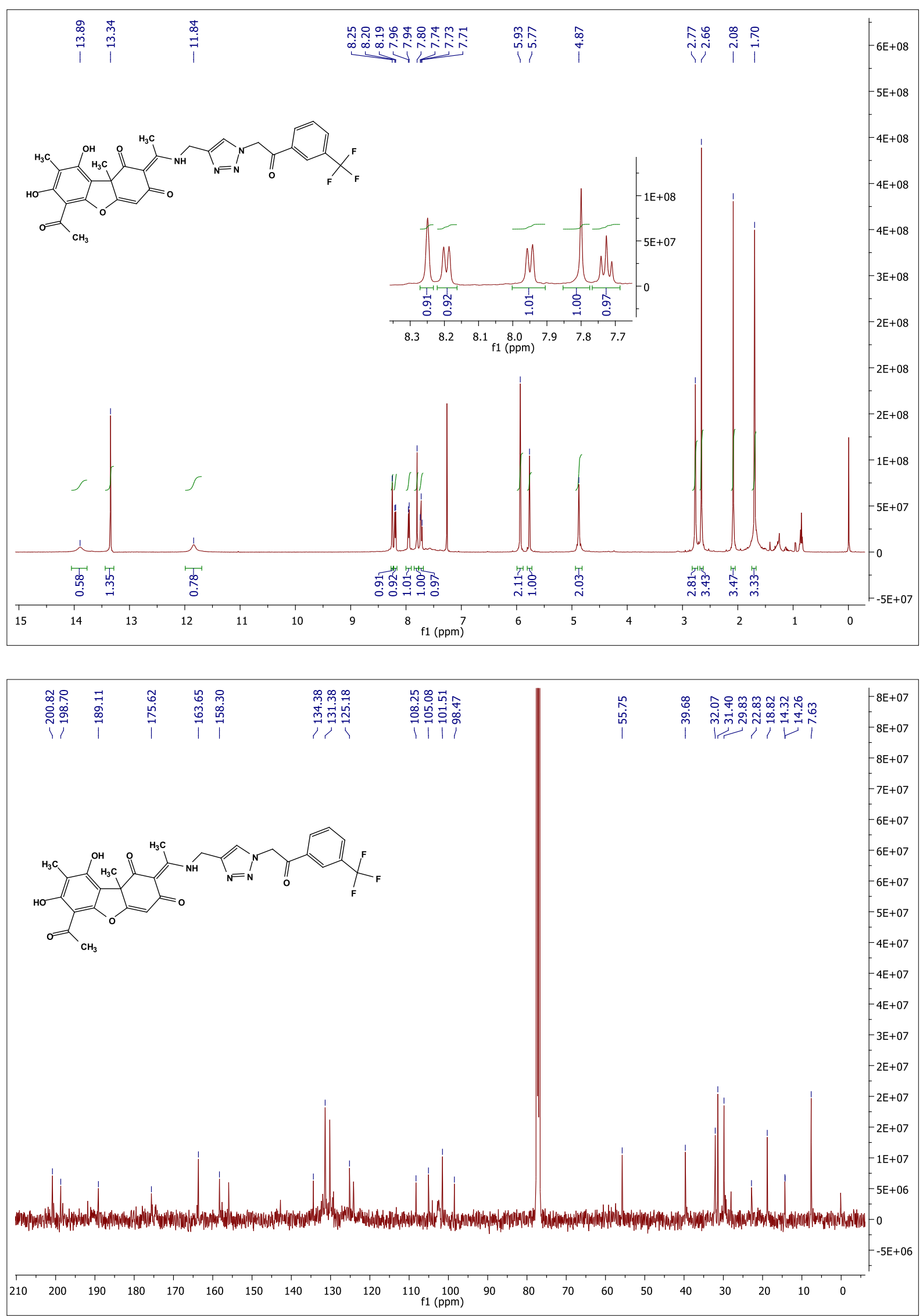
${ }^{1} \mathrm{H} \&{ }^{13} \mathrm{C}$ NMR for compound 19
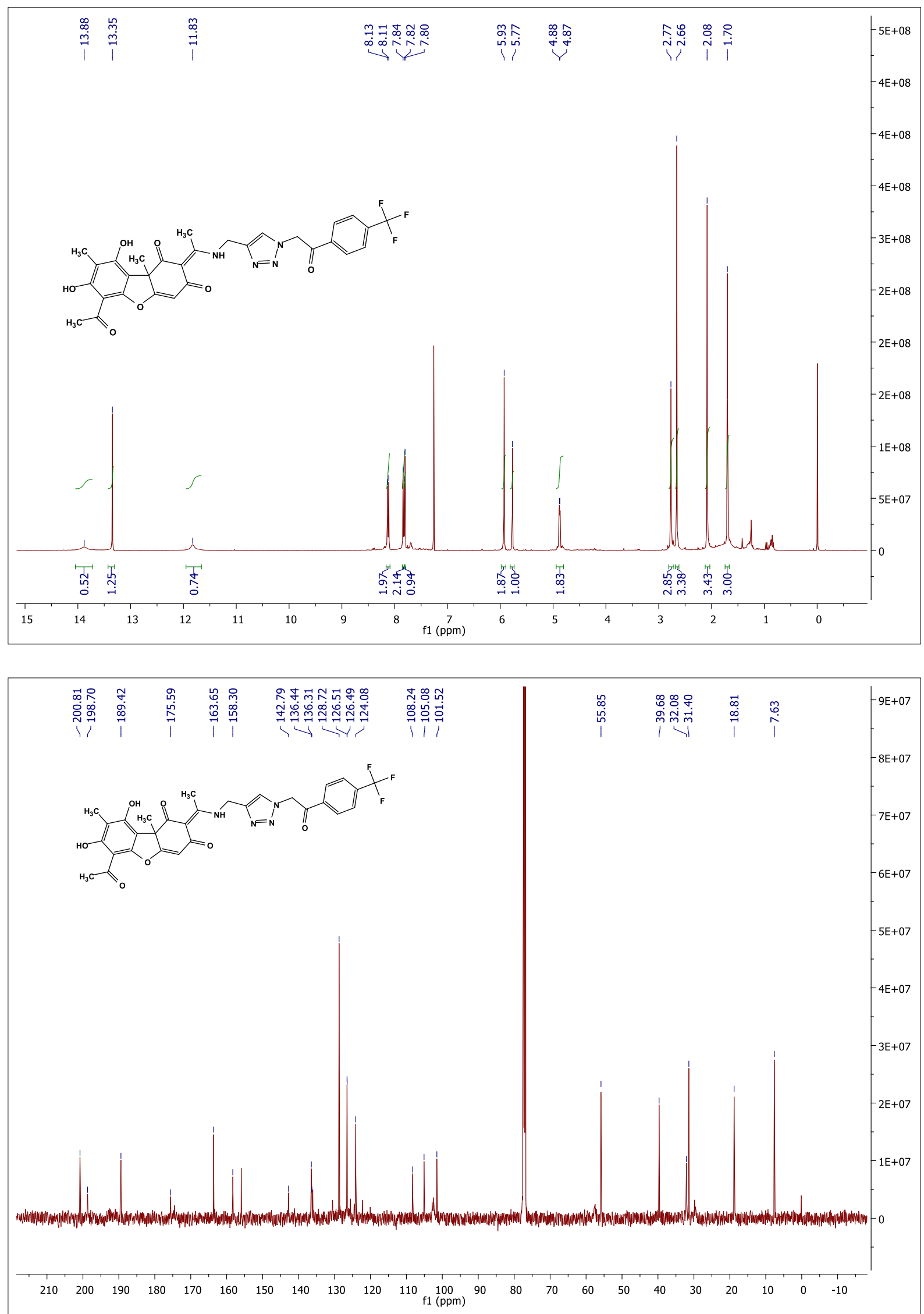
${ }^{1} \mathrm{H} \&{ }^{13} \mathrm{C}$ NMR for compound 20
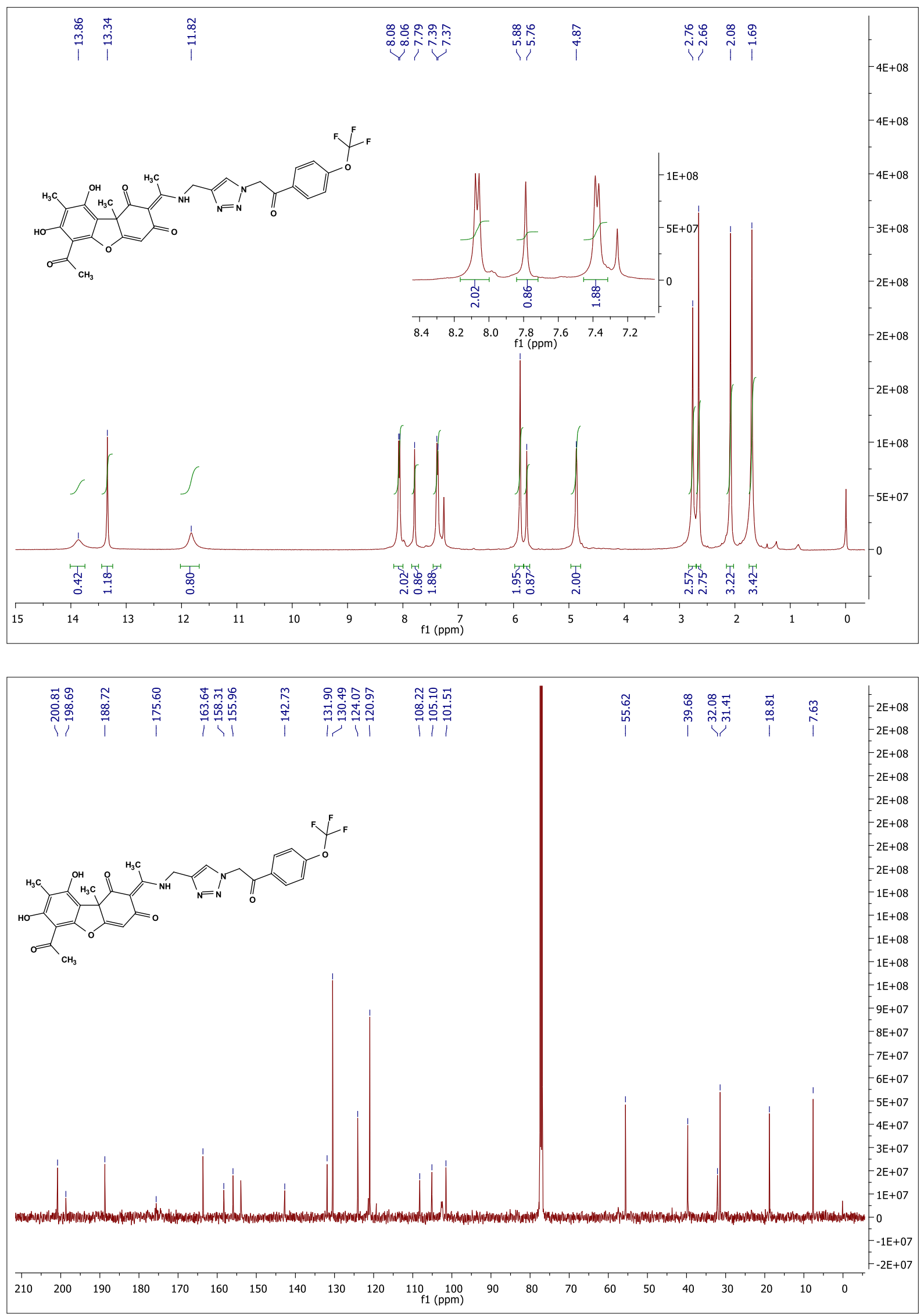
${ }^{1} \mathrm{H} \&{ }^{13} \mathrm{C}$ NMR for compound 21
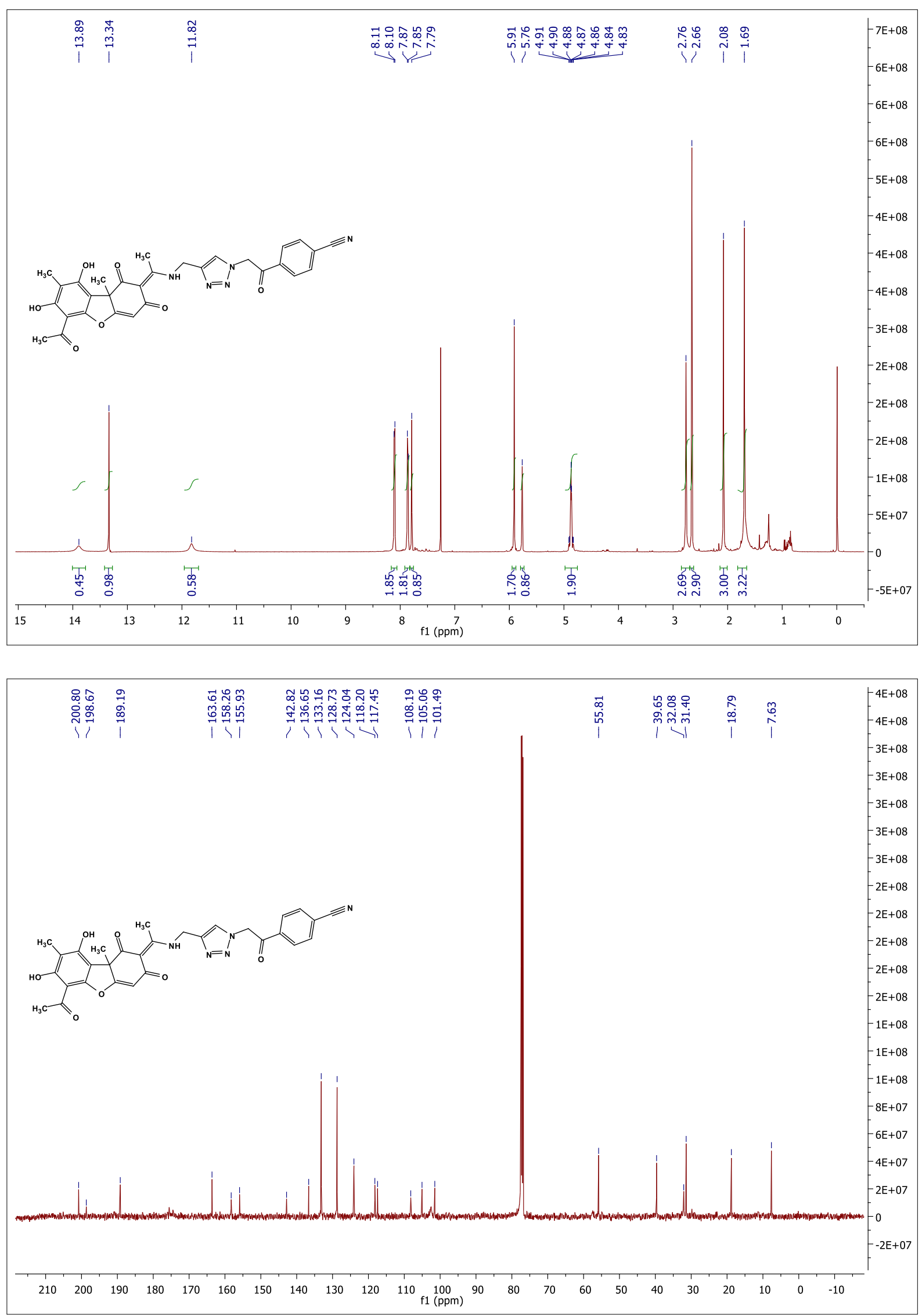
${ }^{1} \mathrm{H} \&{ }^{13} \mathrm{C}$ NMR for compound 22
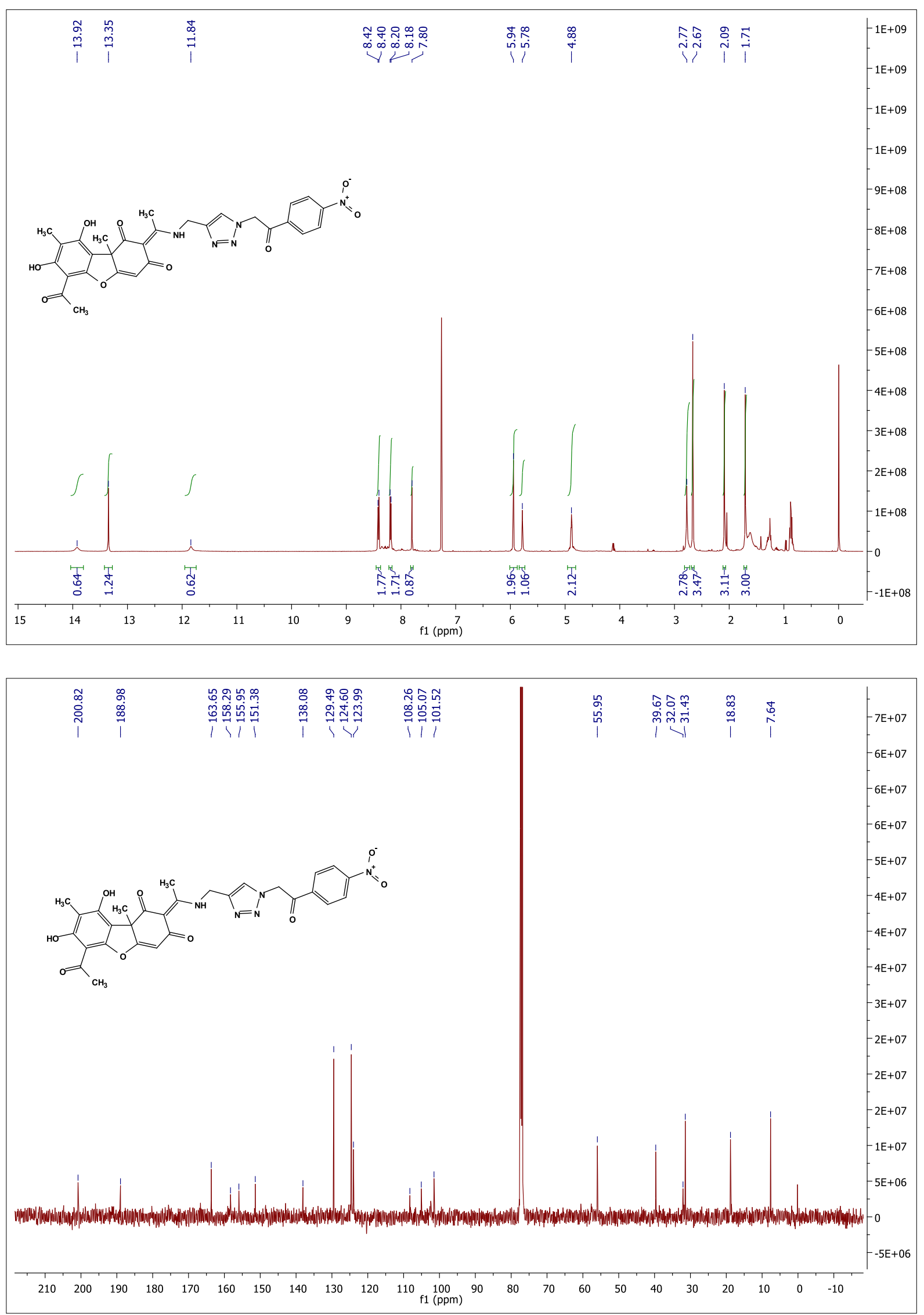
${ }^{1} \mathrm{H} \&{ }^{13} \mathrm{C}$ NMR for compound 23
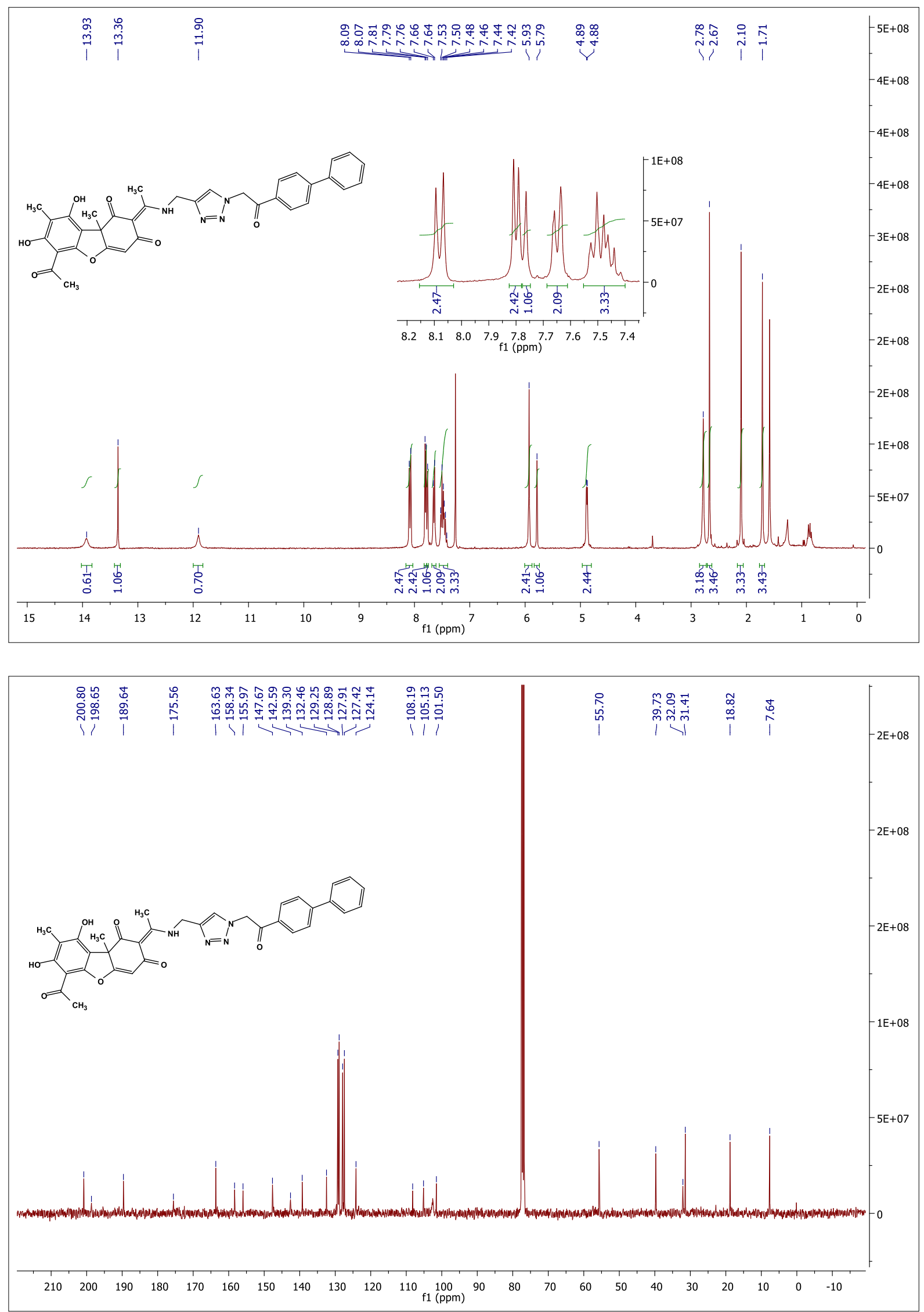
${ }^{1} \mathrm{H} \&{ }^{13} \mathrm{C}$ NMR for compound 24
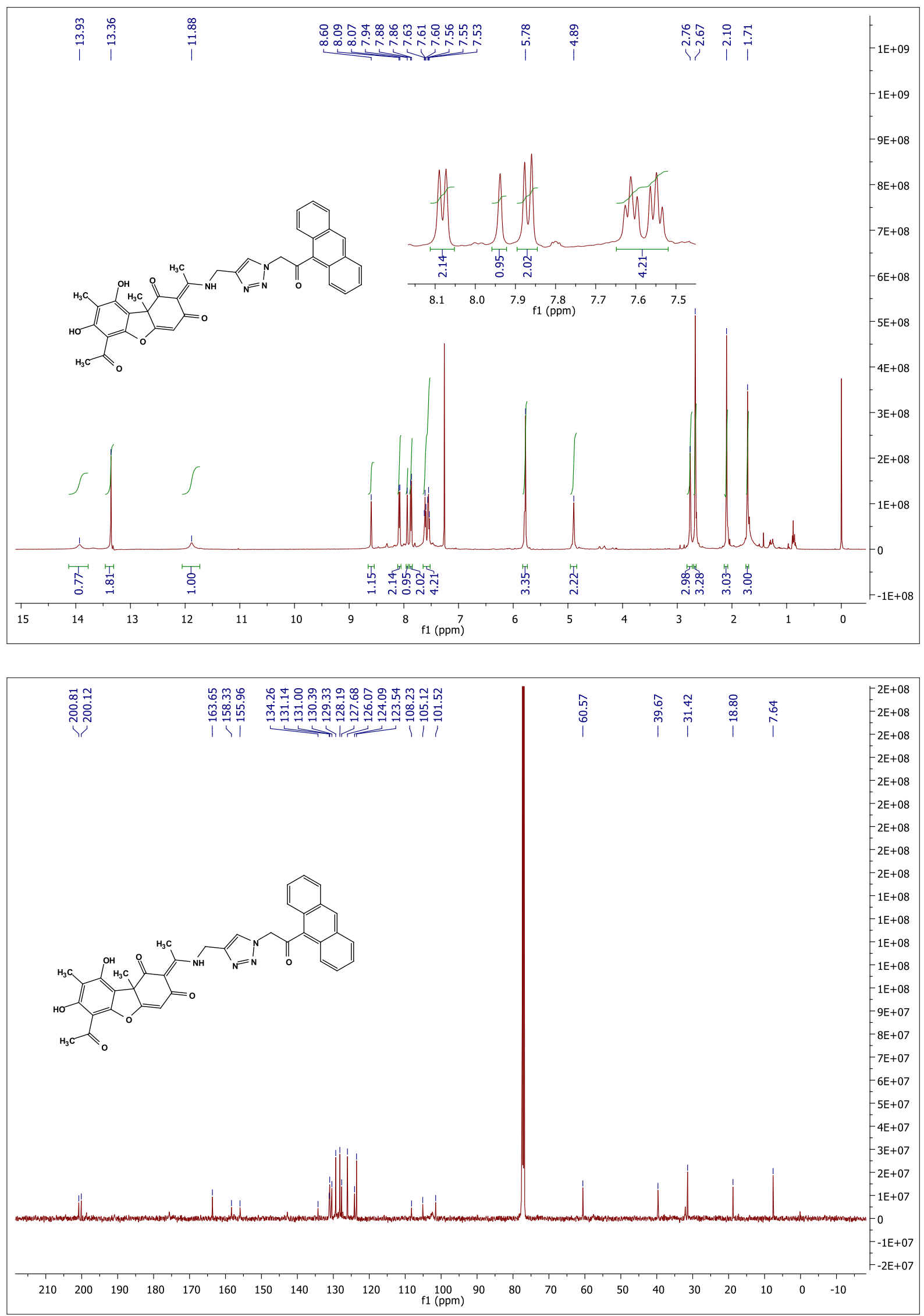
${ }^{1} \mathrm{H} \&{ }^{13} \mathrm{C}$ NMR for compound 25
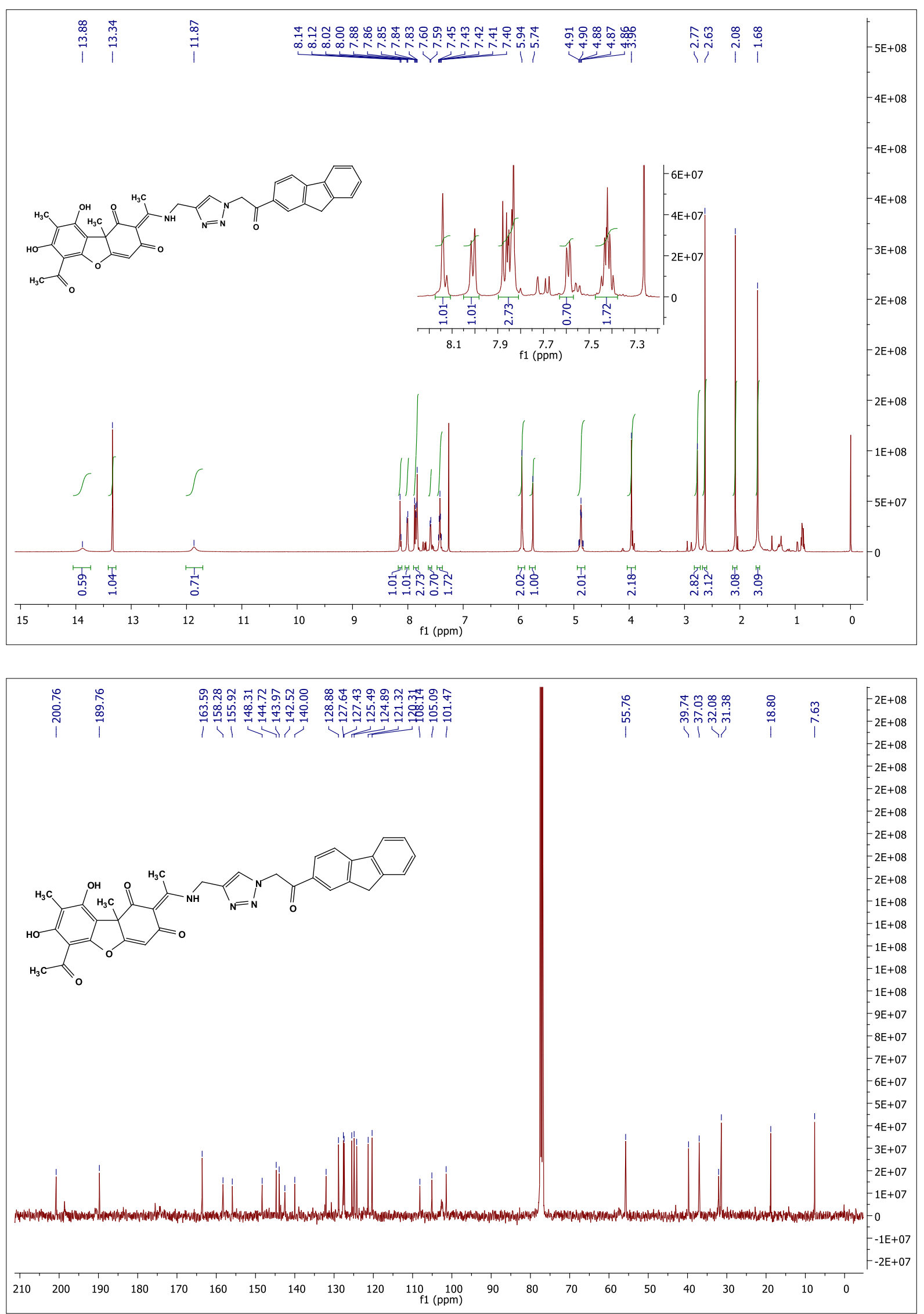
${ }^{1} \mathrm{H} \&{ }^{13} \mathrm{C}$ NMR for compound 26
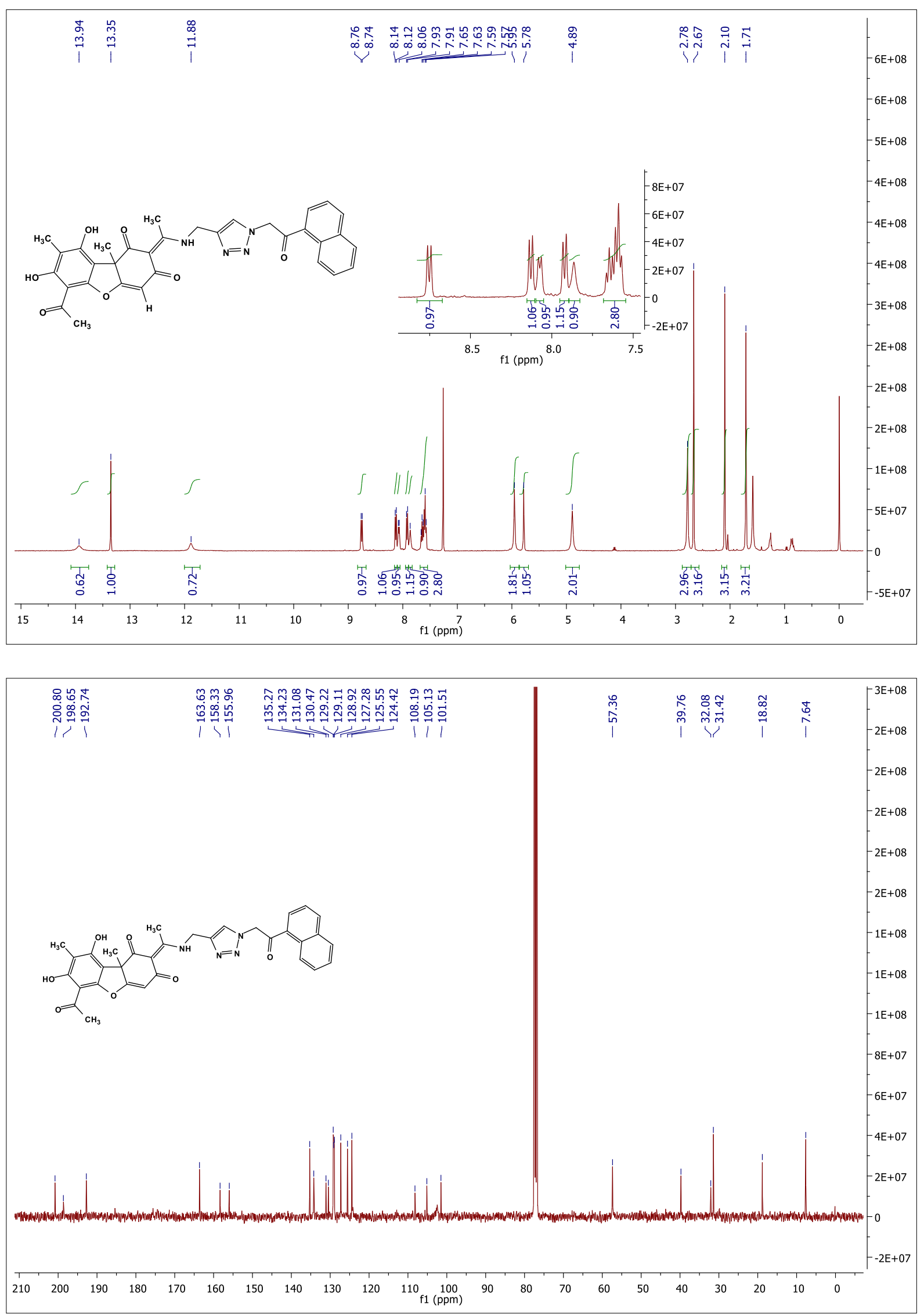
${ }^{1} \mathrm{H} \&{ }^{13} \mathrm{C}$ NMR for compound 27
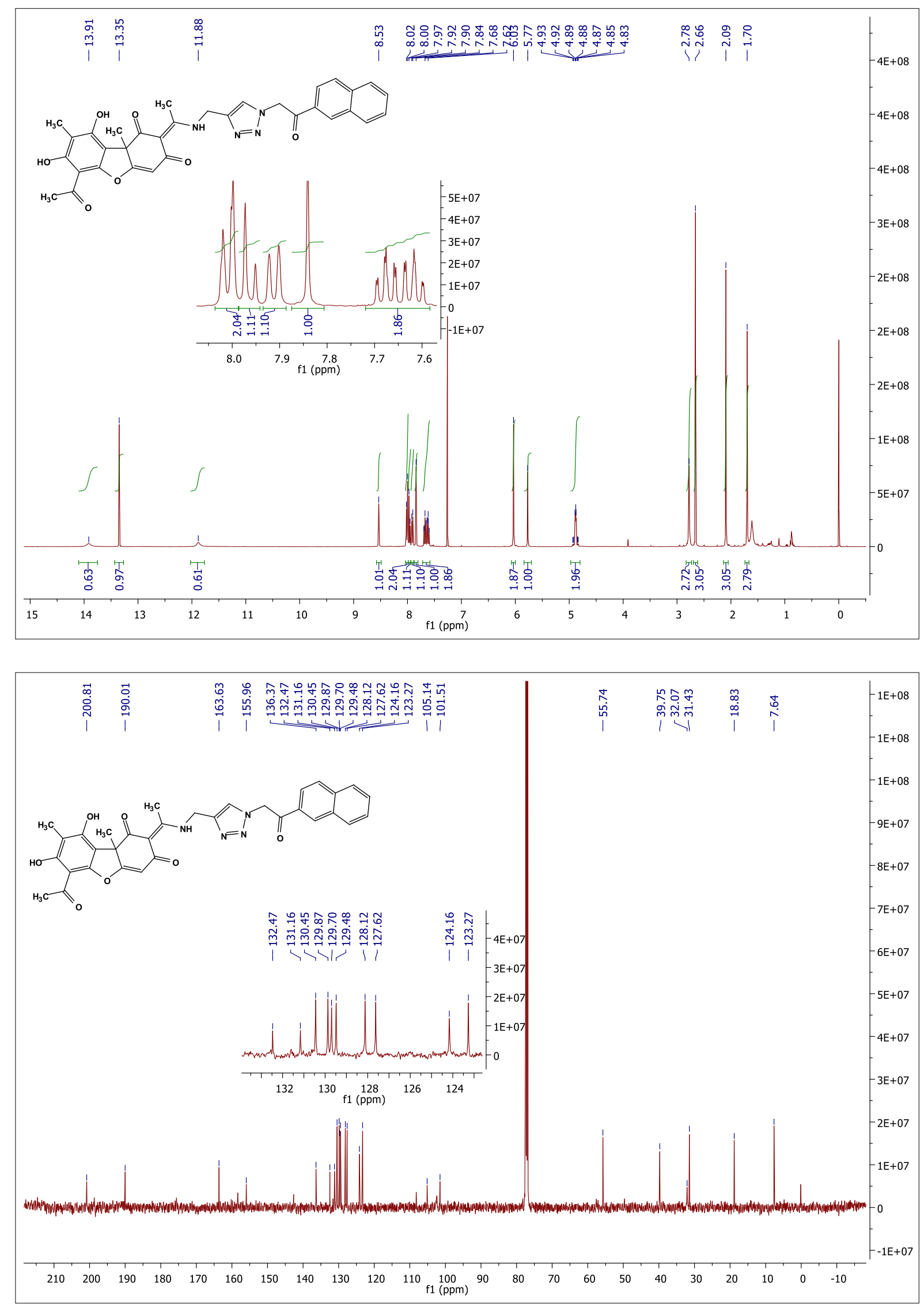
${ }^{1} \mathrm{H} \&{ }^{13} \mathrm{C}$ NMR for compound 28
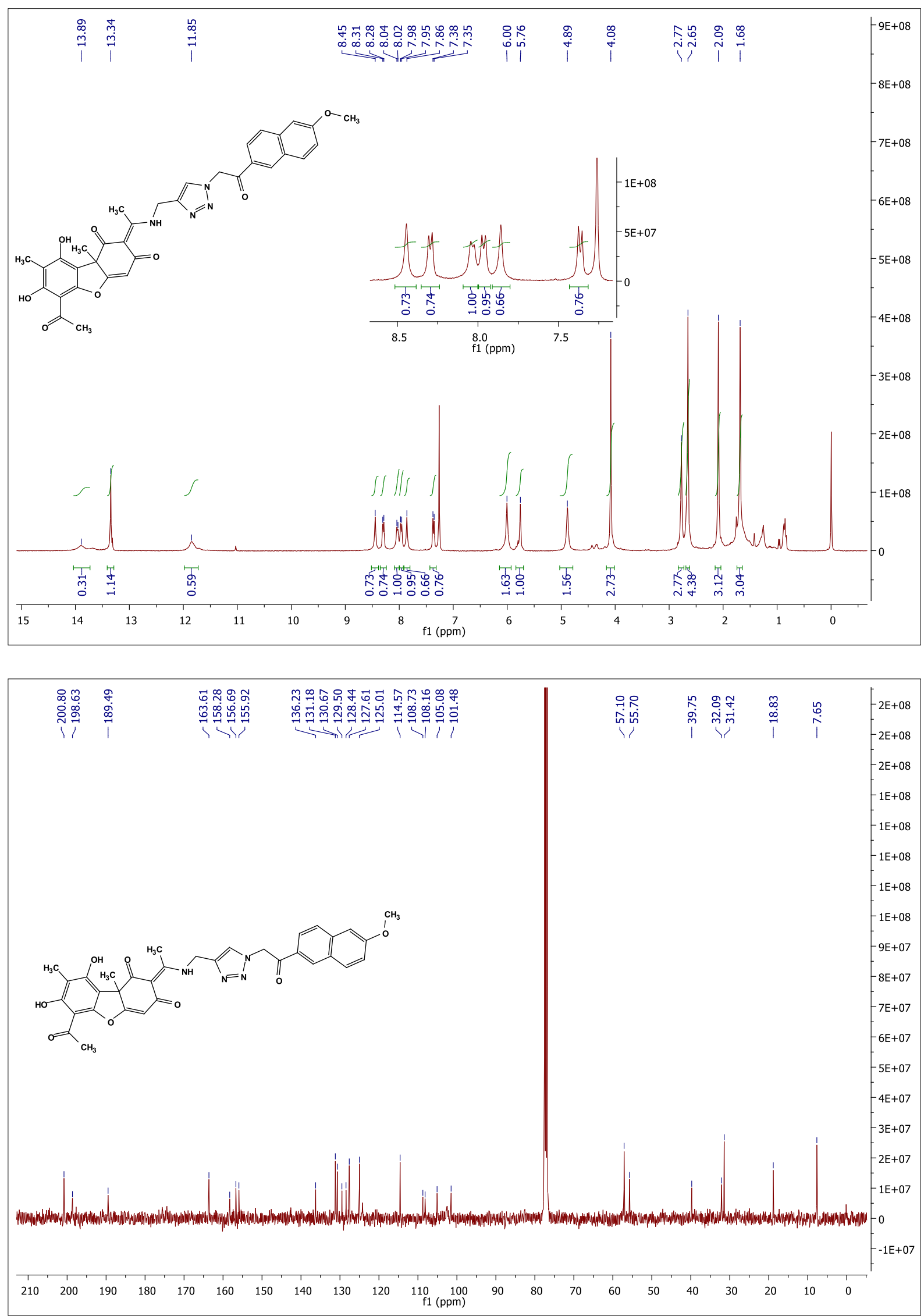
${ }^{1} \mathrm{H} \&{ }^{13} \mathrm{C}$ NMR for compound 29
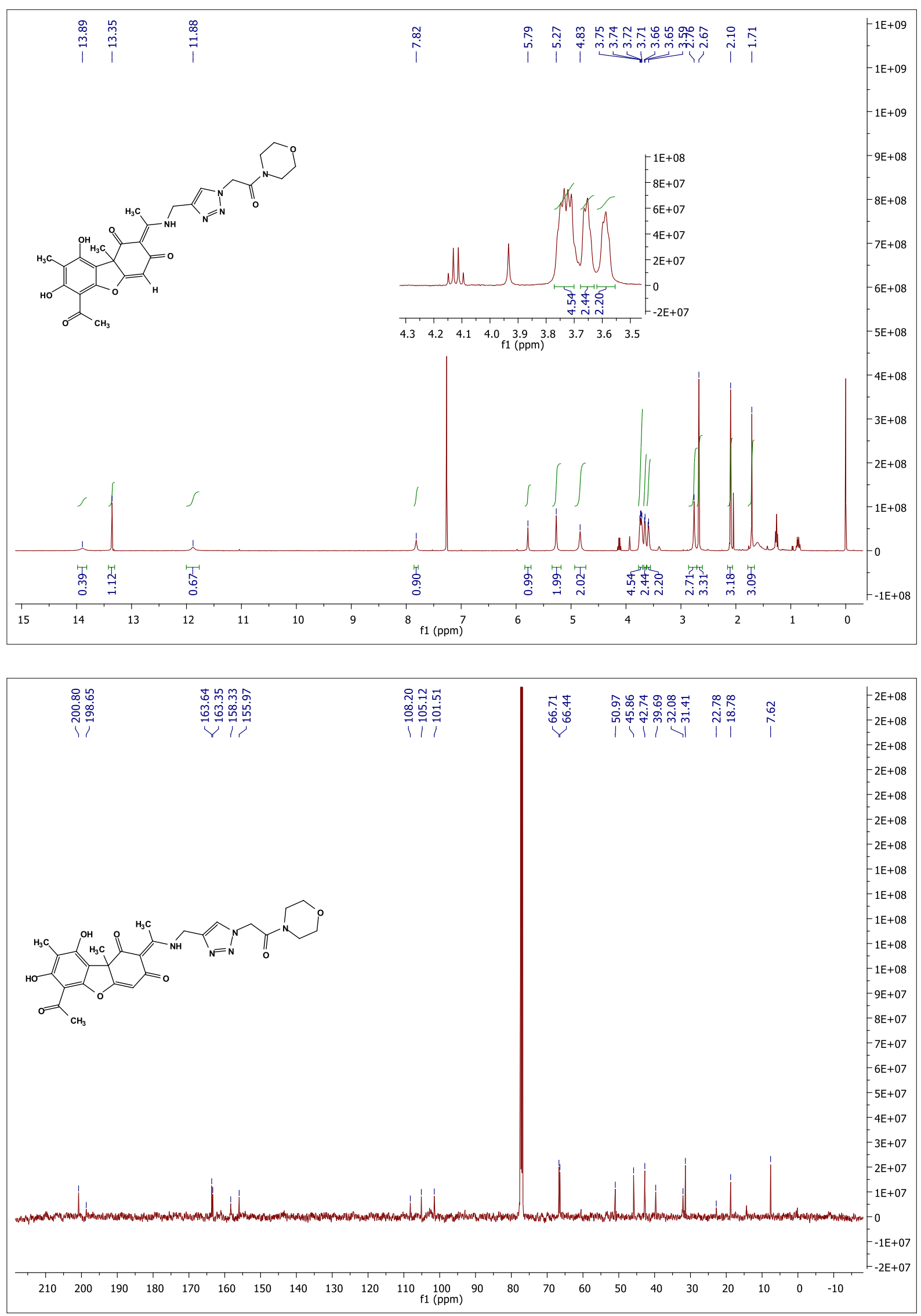
${ }^{1} \mathrm{H} \&{ }^{13} \mathrm{C}$ NMR for compound 30
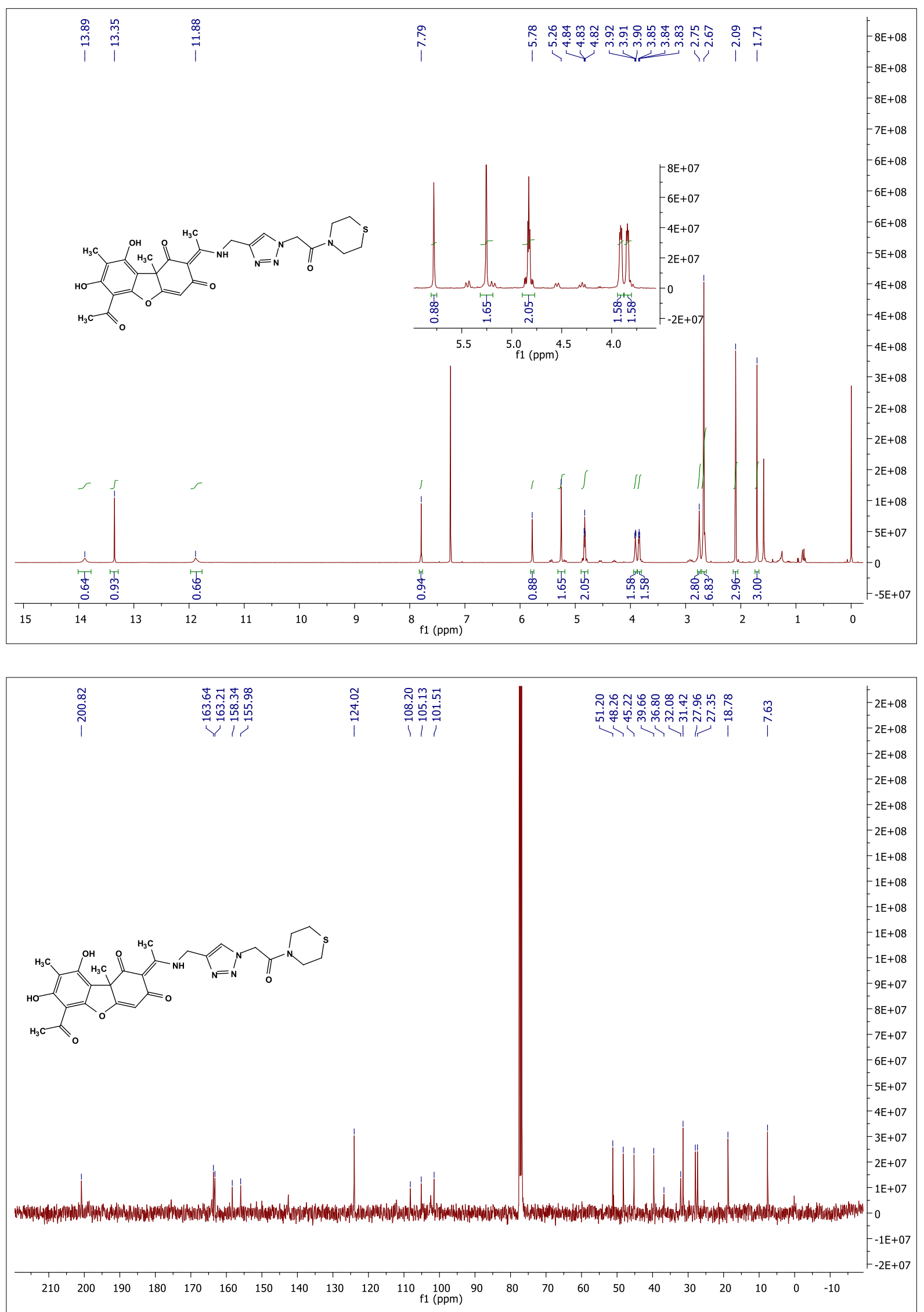
${ }^{1} \mathrm{H} \&{ }^{13} \mathrm{C}$ NMR for compound 31
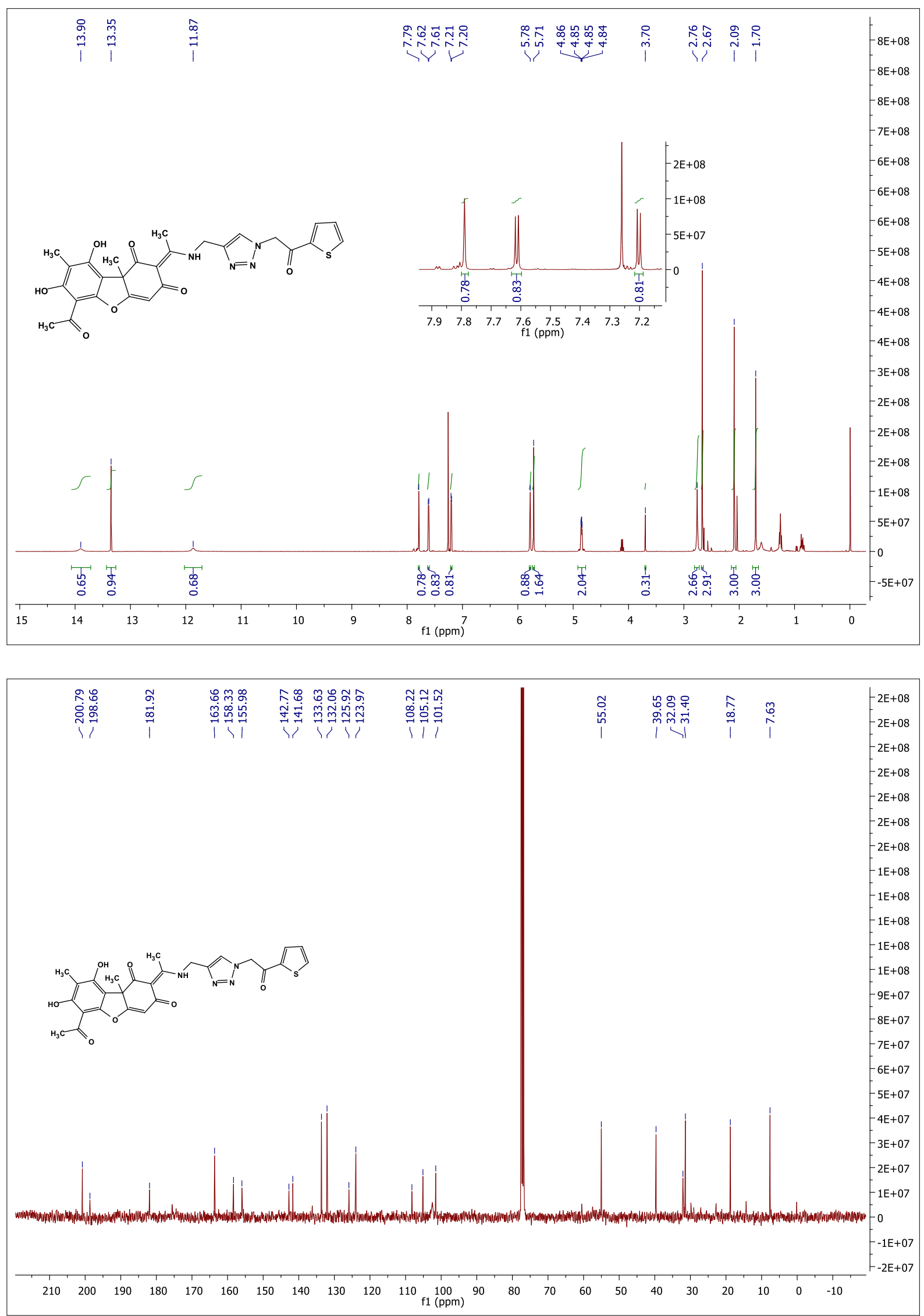
${ }^{1} \mathrm{H} \&{ }^{13} \mathrm{C}$ NMR for compound 32
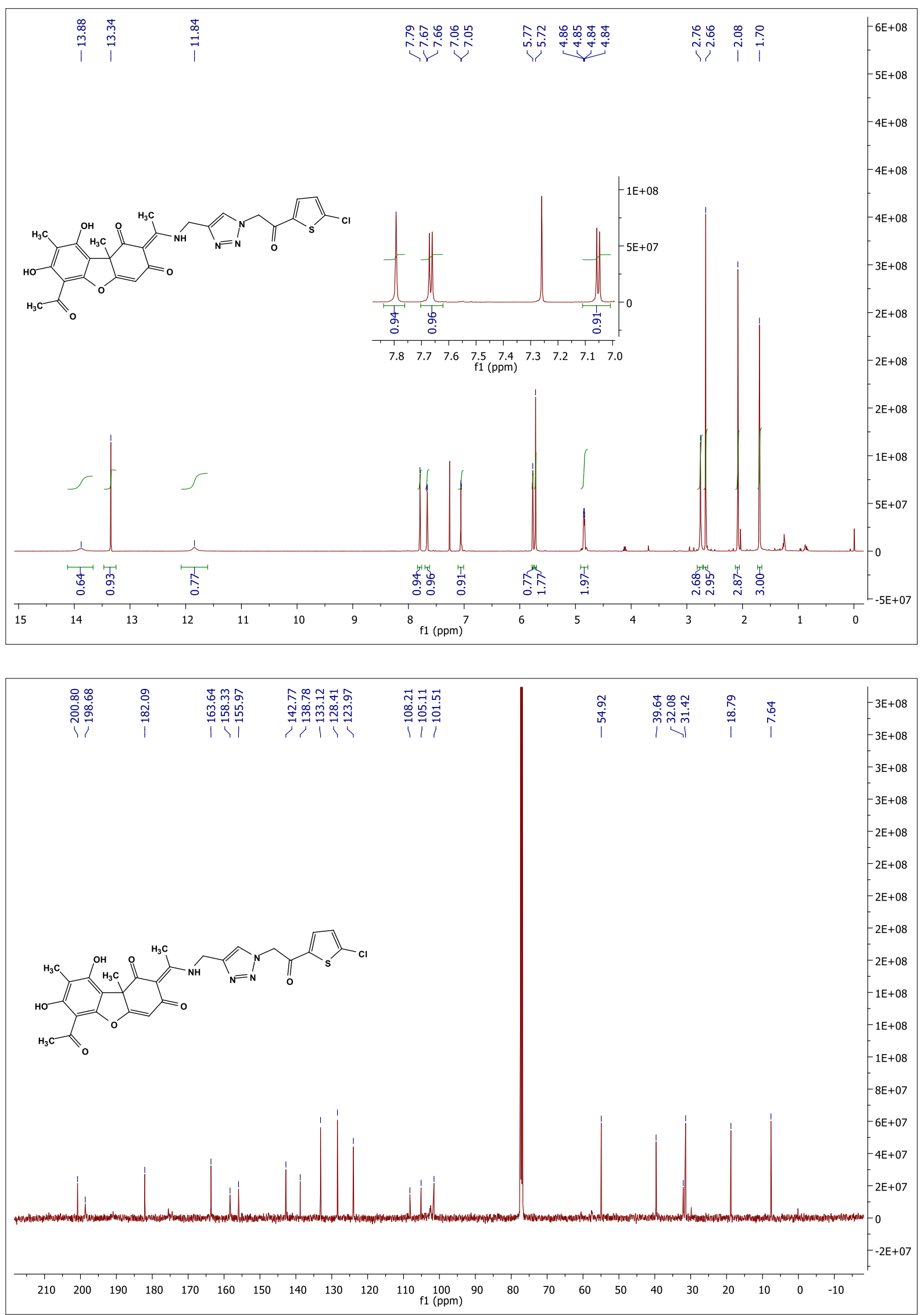
${ }^{1} \mathrm{H} \&{ }^{13} \mathrm{C}$ NMR for compound 33
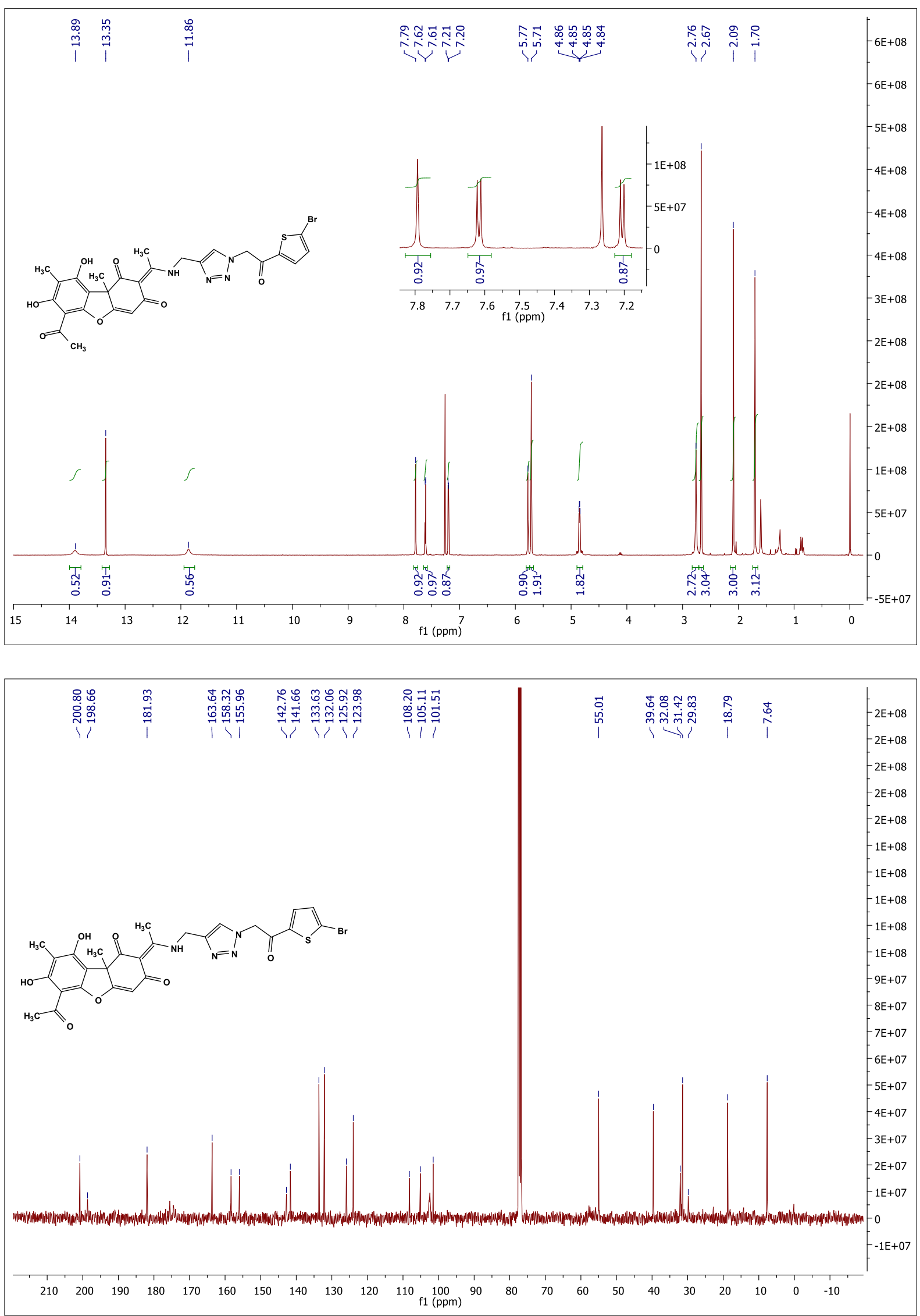
${ }^{1} \mathrm{H} \&{ }^{13} \mathrm{C}$ NMR for compound 34
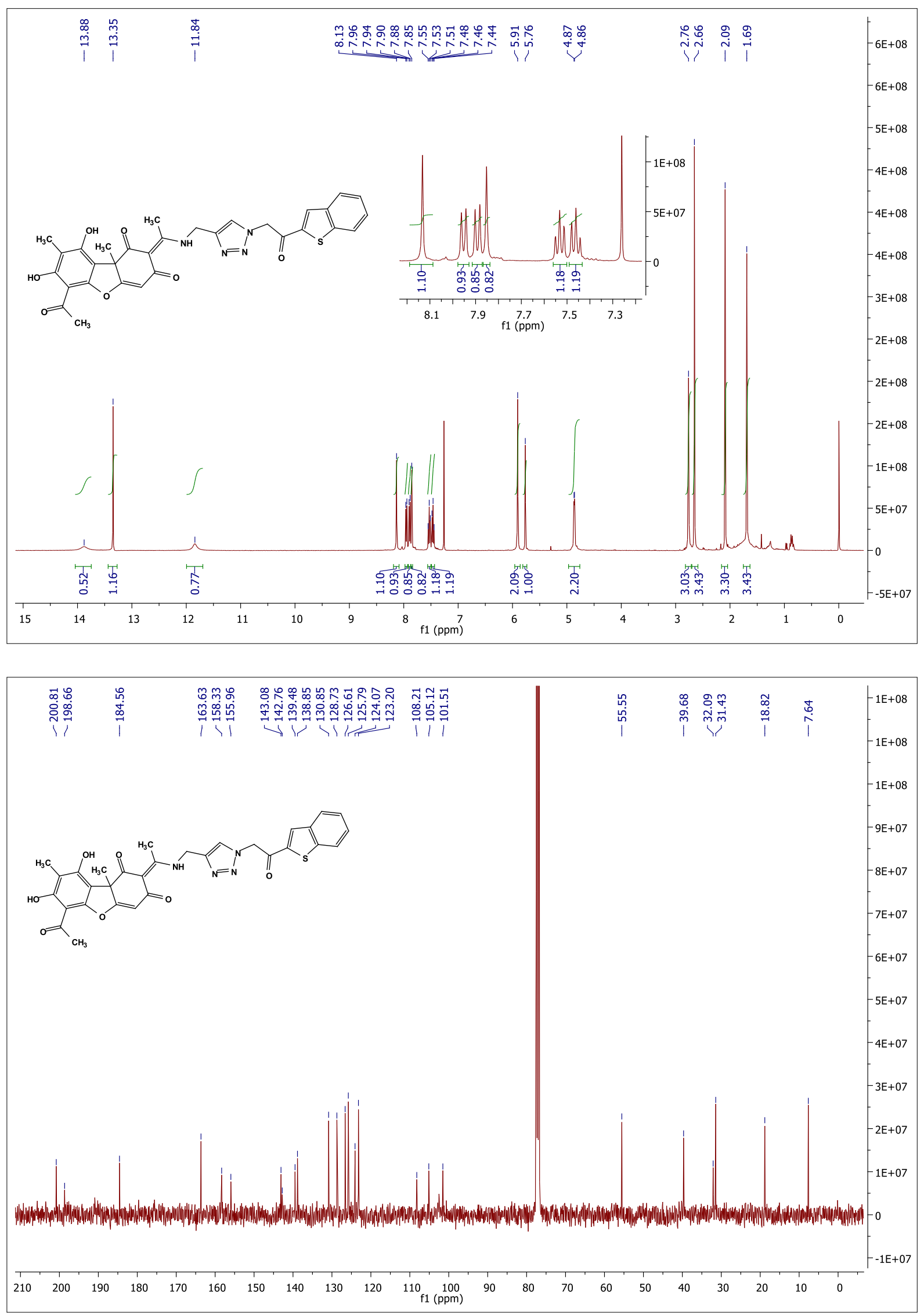
${ }^{1} \mathrm{H} \&{ }^{13} \mathrm{C}$ NMR for compound 35
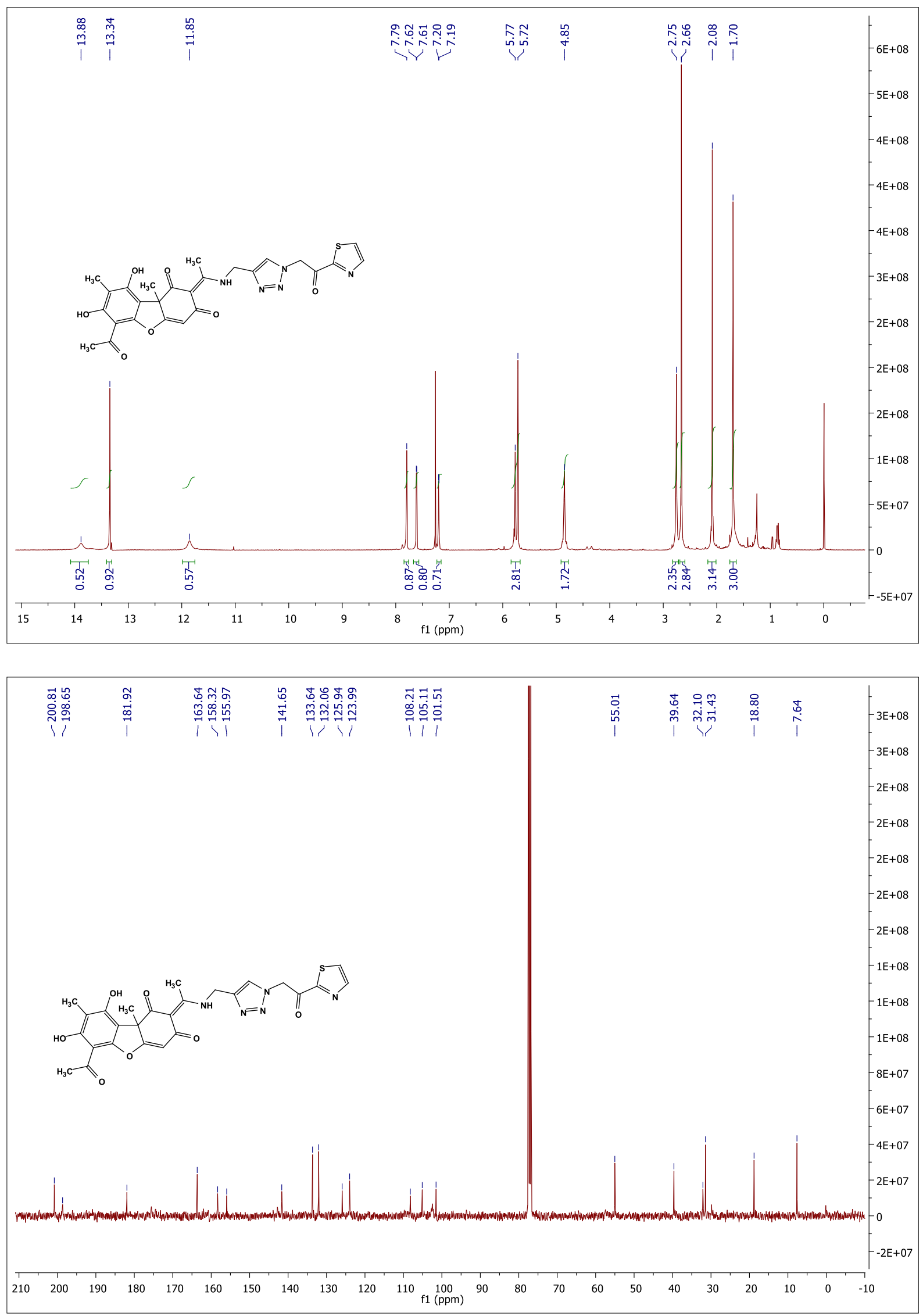
${ }^{1} \mathrm{H} \&{ }^{13} \mathrm{C}$ NMR for compound 36
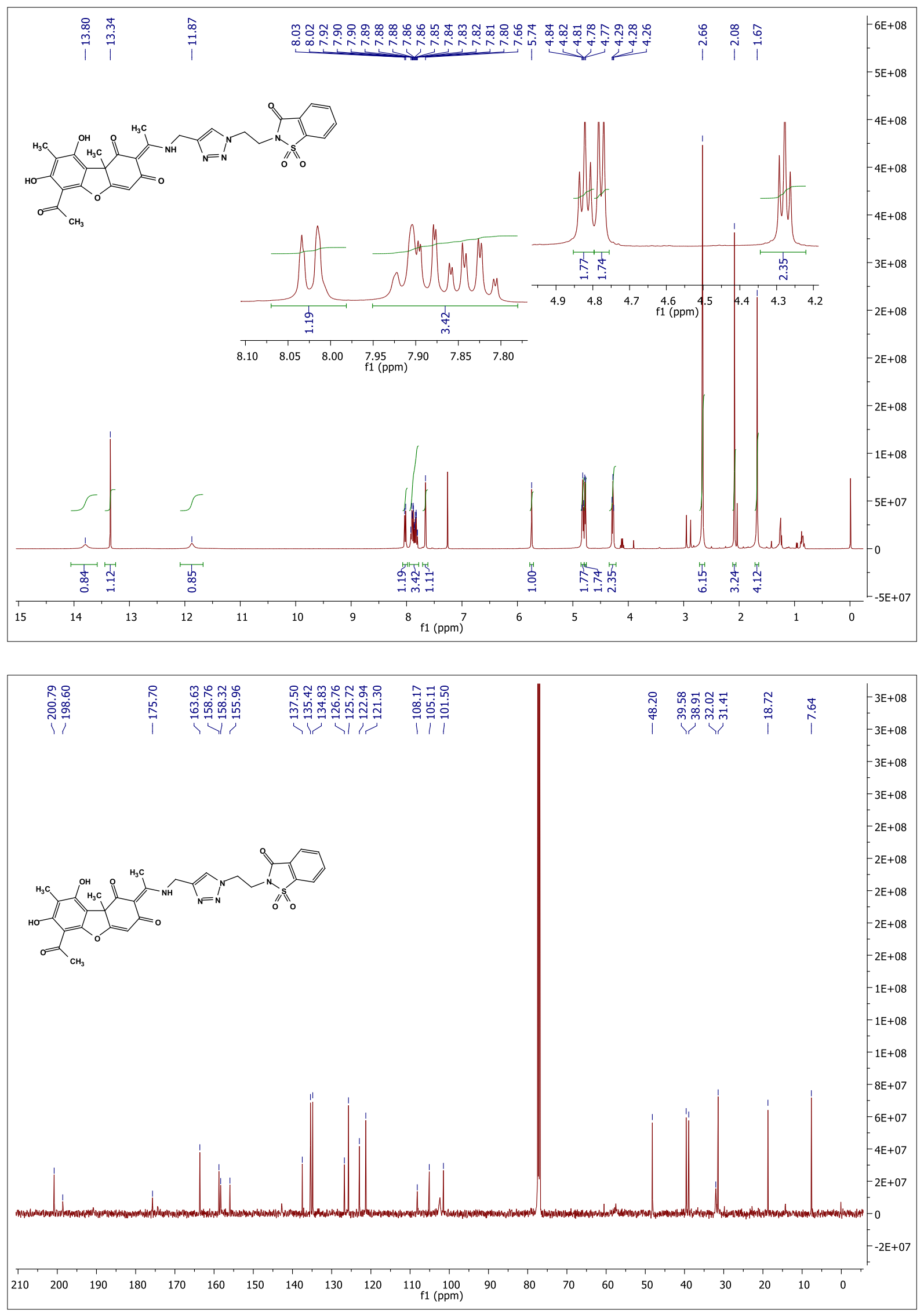
${ }^{1} \mathrm{H} \&{ }^{13} \mathrm{C}$ NMR for compound 37
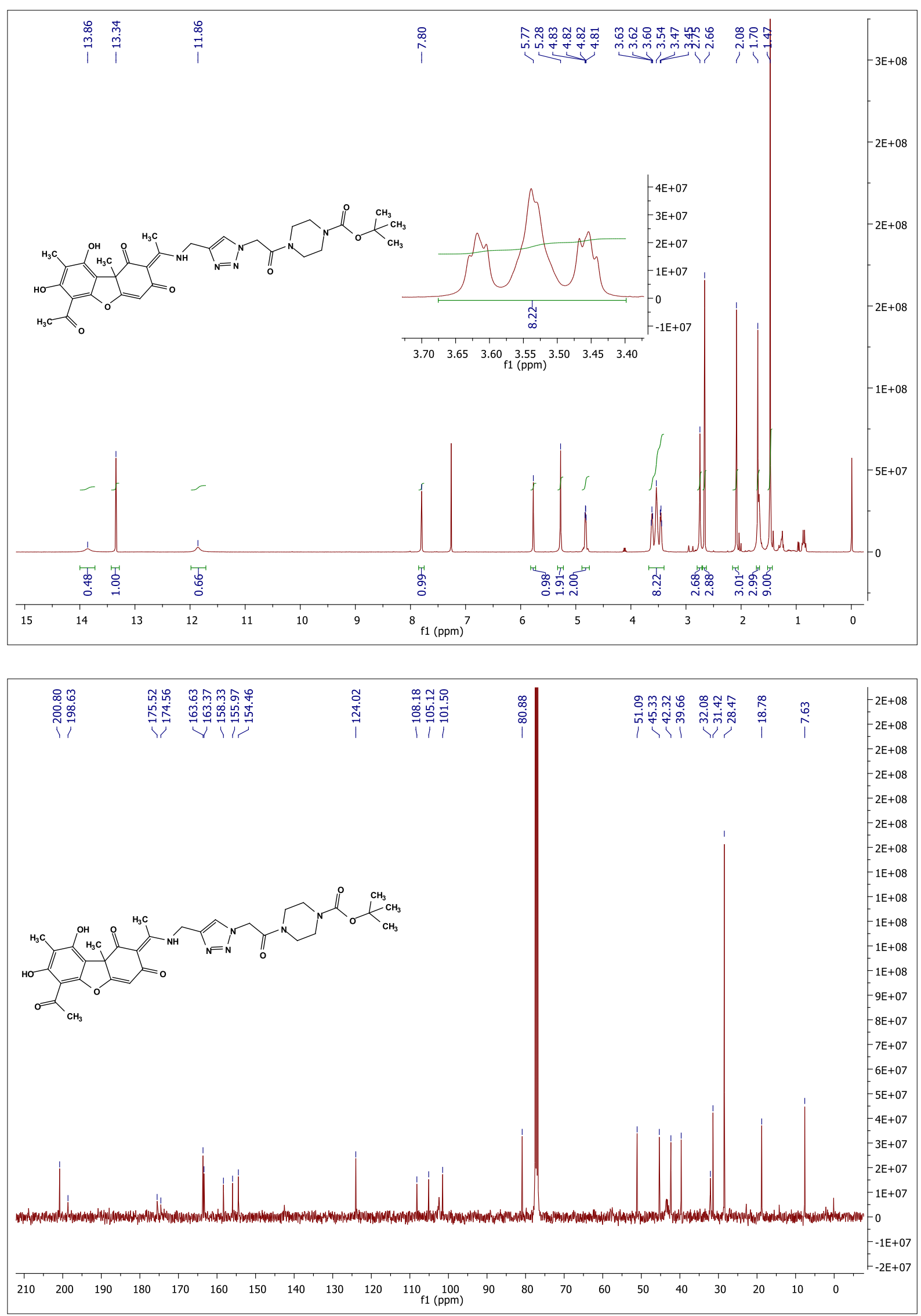
${ }^{1} \mathrm{H} \&{ }^{13} \mathrm{C}$ NMR for compound 38
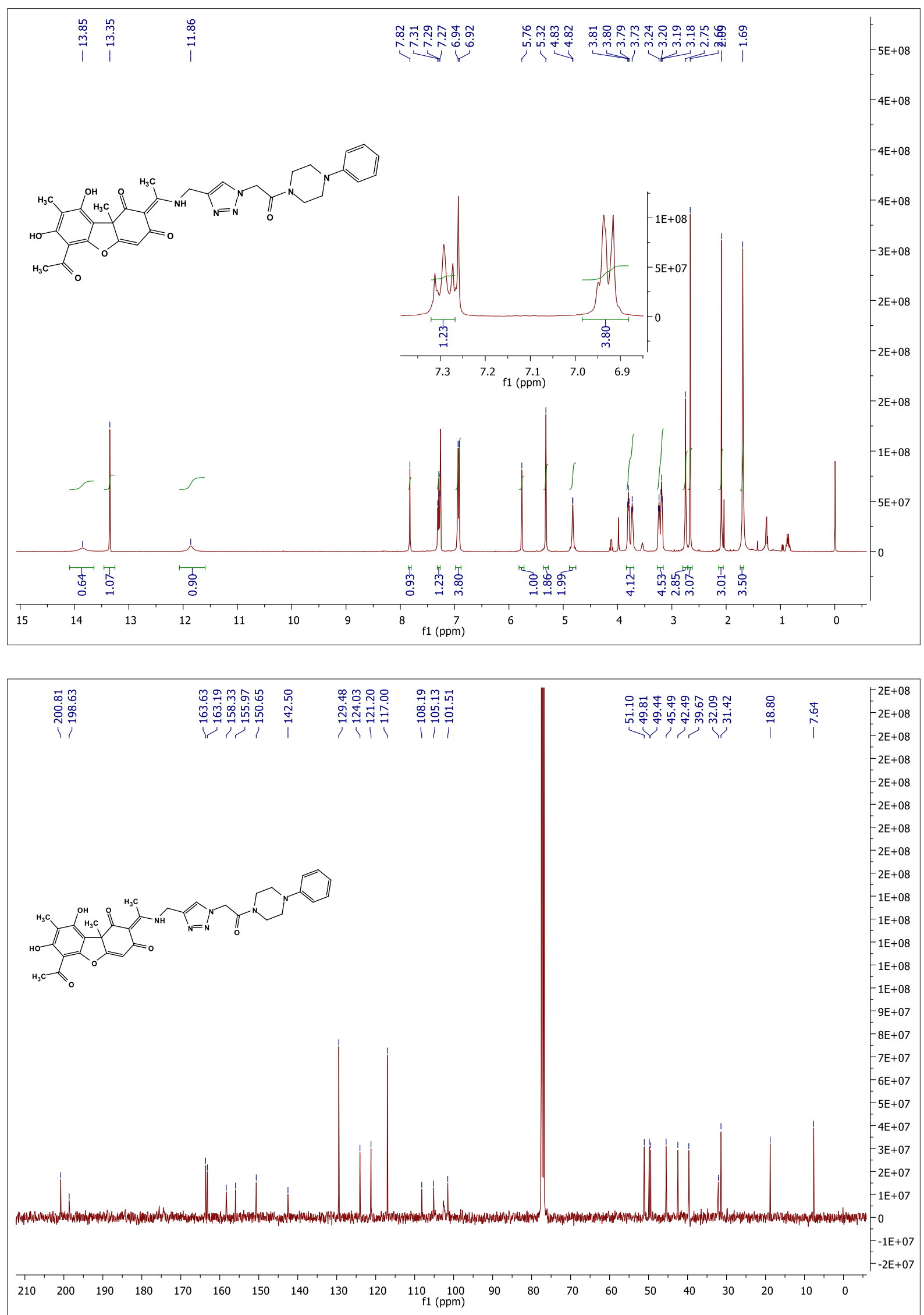
${ }^{1} \mathrm{H} \&{ }^{13} \mathrm{C}$ NMR for compound 39
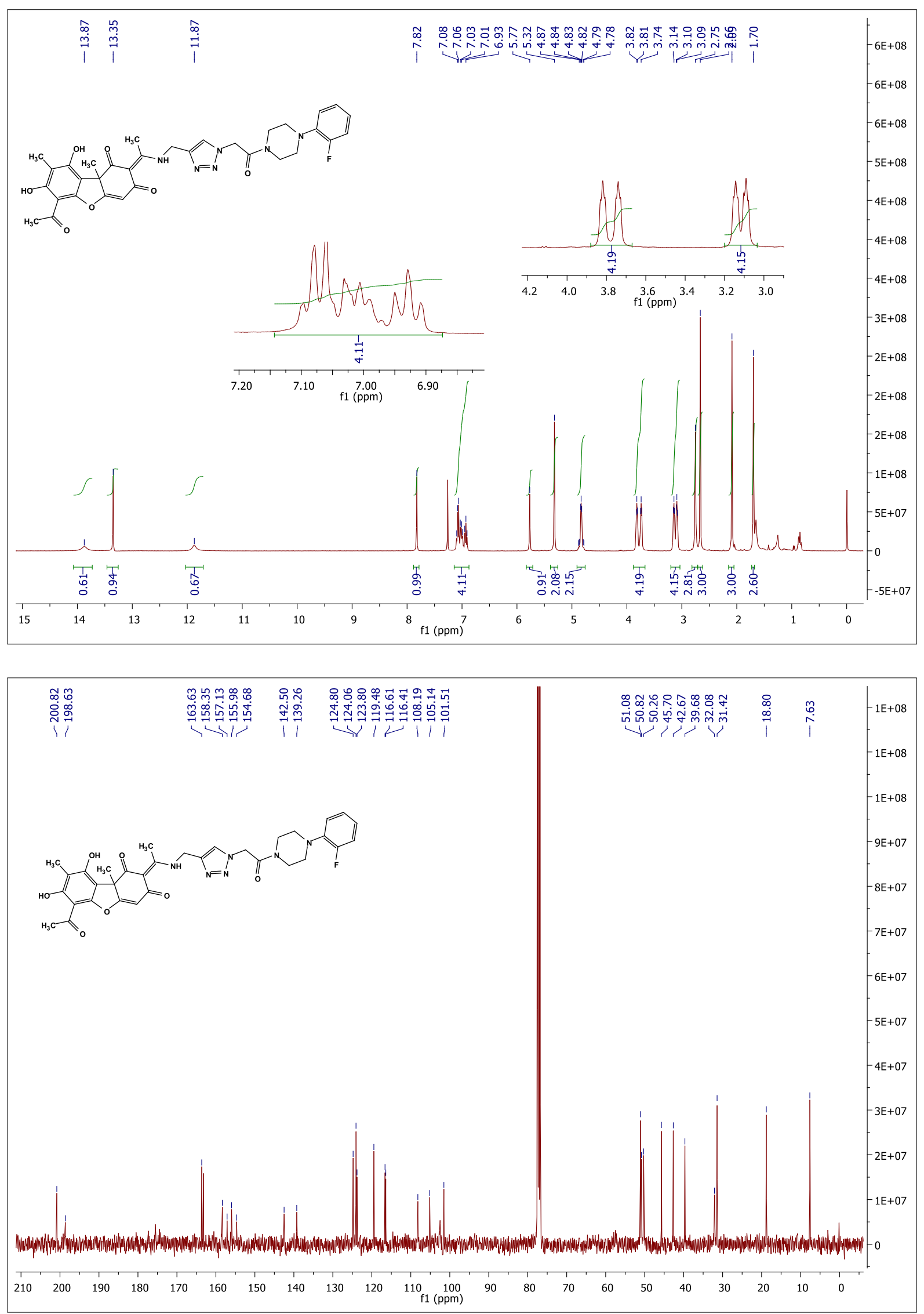
${ }^{1} \mathrm{H} \&^{13} \mathrm{C}$ NMR for compound 40
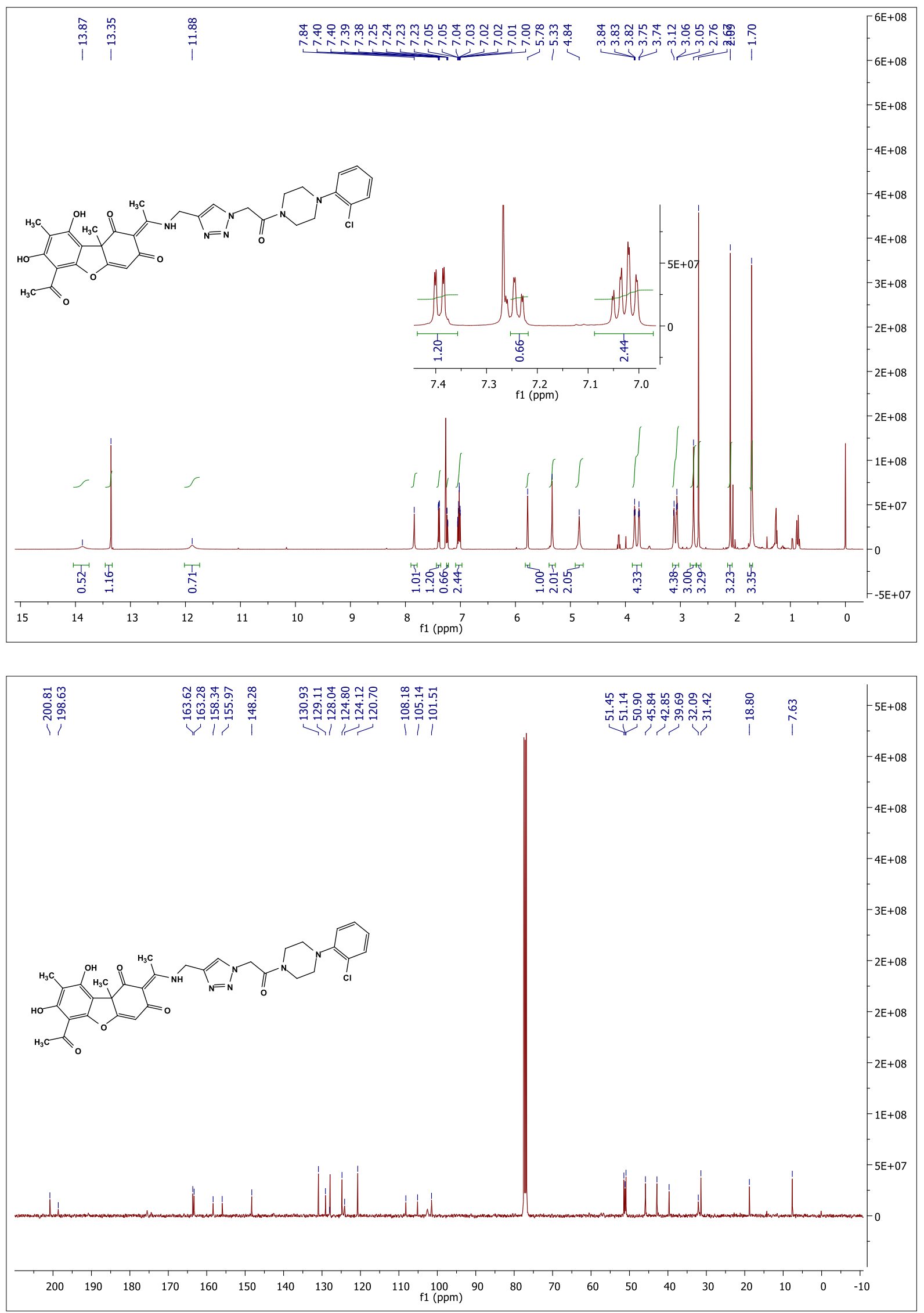
${ }^{1} \mathrm{H} \&{ }^{13} \mathrm{C}$ NMR for compound 41
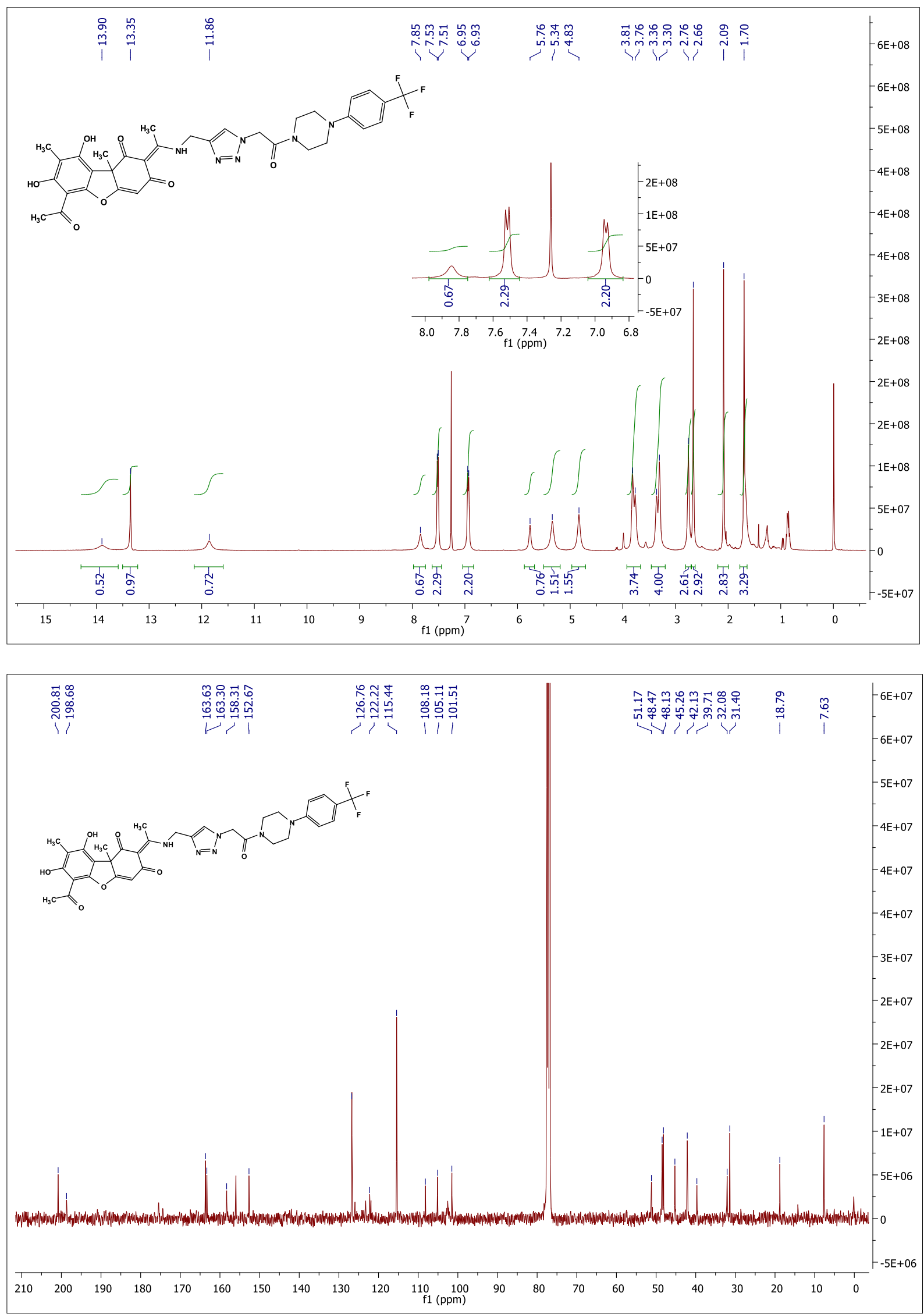
${ }^{1} \mathrm{H} \&{ }^{13} \mathrm{C}$ NMR for compound 42
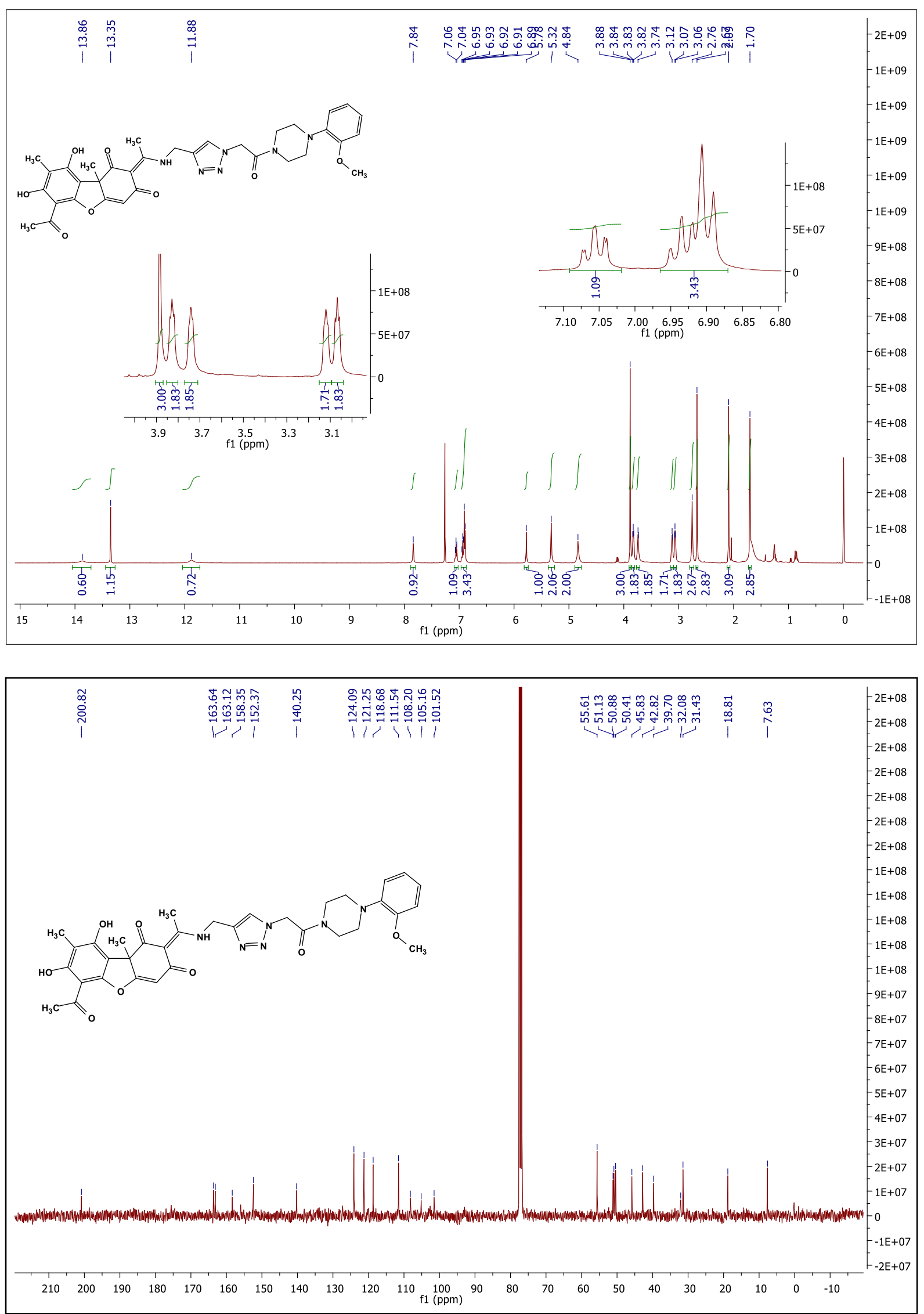
${ }^{1} \mathrm{H} \&{ }^{13} \mathrm{C}$ NMR for compound 43
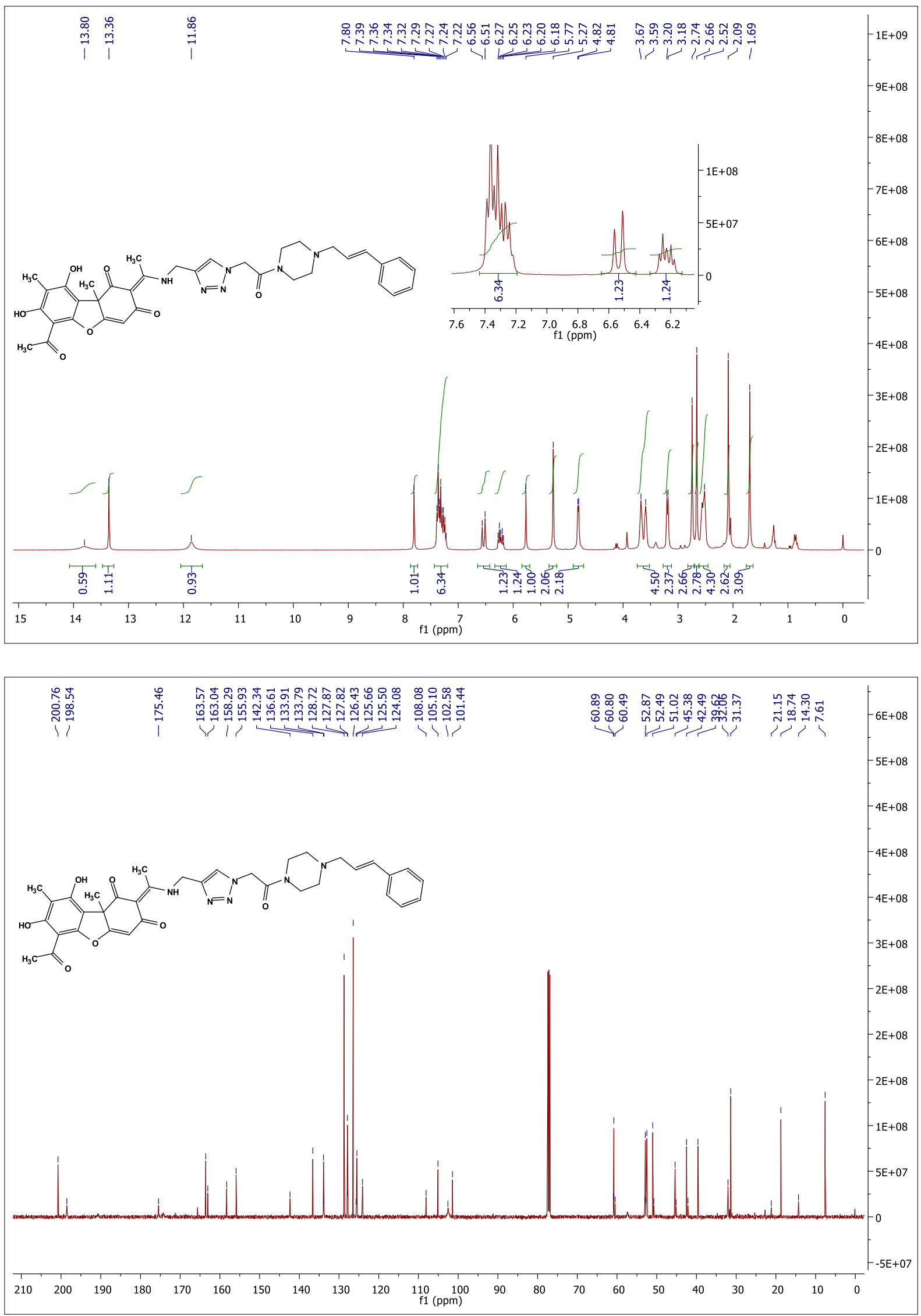
${ }^{1} \mathrm{H} \&{ }^{13} \mathrm{C}$ NMR for compound 44
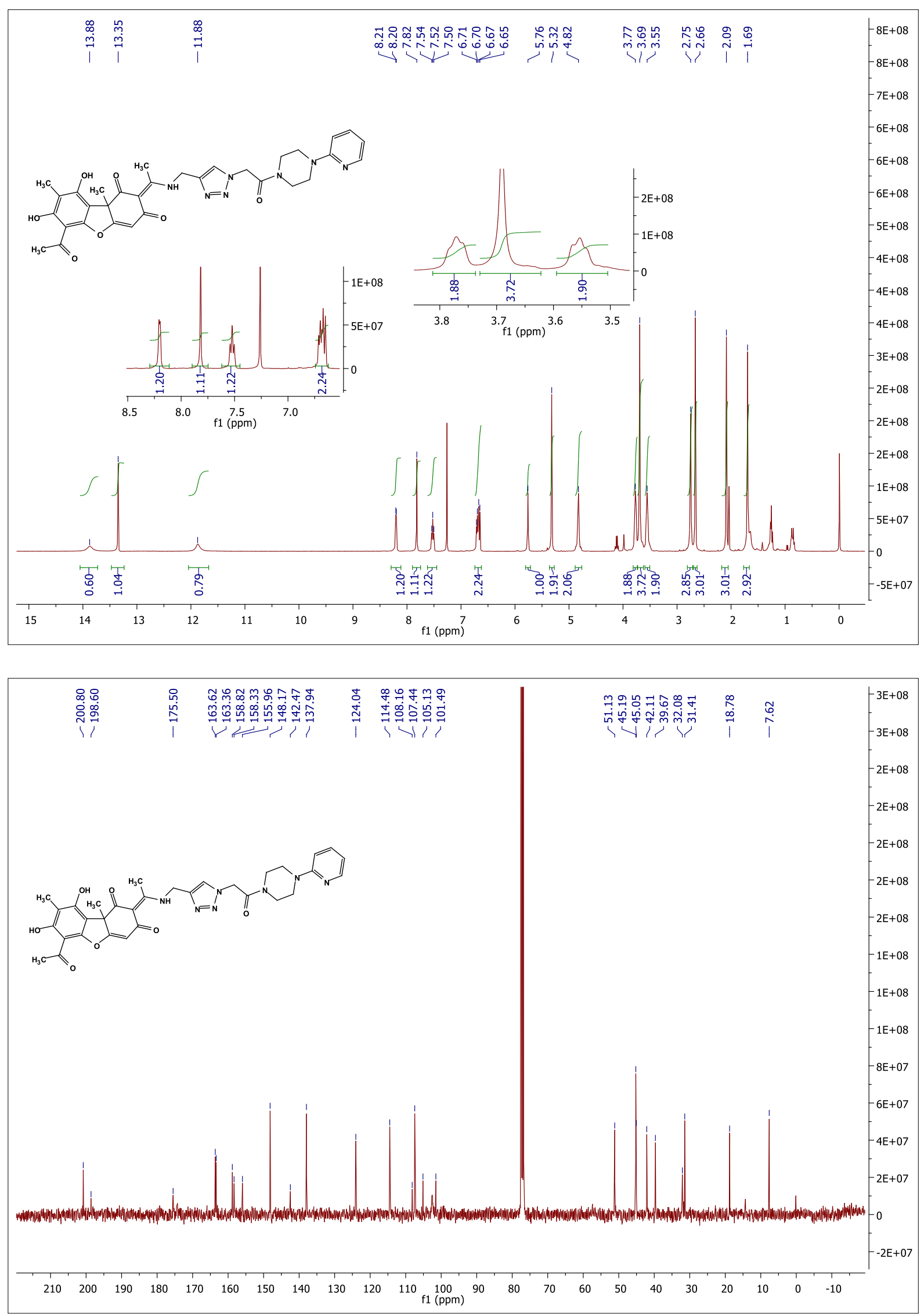


\section{- References}

1. (a) Bruker (2016) APEX3, SAINT and SADABS. Bruker AXS, Inc., Madison, Wisconsin, USA. (b) Sheldrick, G. M. Acta Crystallogr. 2015, C71, 3-8.

2. Hossain, A.; Vidyasagar, A.; Eichinger, C.; Lankes, C.; Phan, J.; Rehbein, J.; Reiser, O. Angew. Chem. Int. Ed Engl. 2018, 57, 8288-8292.

3. Singh P. N. D.; Mandel, S. M.; Robinson, R. M.; Zhu, Z.; Franz, R.; Ault, B. S.; Gudmundsdottir, A. D. J. Org. Chem. 2003, 68, 7951-7960.

4. Weidner, K.; Sun, Z.; Kumagai, N.; Shibasaki, M. Angew. Chem. 2015, 54, 6236-6240.

5. Yu, B.; Wang, S. -Q.; Qi, P. -P.; Yang, D. -X.; Tang, K.; Liu, H. -M. Eur. J. Med. Chem. 2016, 124, 350-360.

6. Chandra, A.; Parida, K. N.; Moorthy, J. N. Tetrahedron. 2017, 73, 5827-5832.

7. Murar, M.; Dobias, J.; Sramel, P.; Addova, G.; Hanquet, G.; Bohac, A. Eur. J. Med. Chem. 2017, 126, 754-761.

8. Wei, W.; Cui, H.; Yue, H.; Yang, D. Green Chem. 2018, 20, 3197-3202.

9. Collins L. A.; Franzblau S. G. Antimicrob. Agents Chemother. 1997, 41, 1004-1009.

10. (a) Betts, J. C.; Lukey, P. T.; Robb, L. C.; McAdam, R. A.; Duncan, K. Mol. Microbiol. 2002, 43, 717-31. (b) Malapati, P.; Krishna, V. S.; Nallangi, R.; Srilakshmi, R. R.; Sriram, D. Eur. J. Med. Chem. 2018, 145, 23-34.

11. Linday, M.E. 1962. Practical Introduction to Microbiology. E and F.N. Spon Ltd., United Kingdom, p. 177.

12. Clinical and Laboratory Standards Institute. Performance Standards for Antimicrobial Susceptibility Tests; Eighteen informational supplements M100-S18 (2008).

13. Javvaji, K.; Begum, G.; Deshpande, S. S.; Rana, R. K.; Misra, S. Chem. Res. Toxicol. 2018, 16, 629-636. 
14. Makane, V. B.; Krishna, V.S.; Krishna, E. V.; Shukla, M.;Mahizhaveni, B.;Misra, S.; Chopra, S.; Sriram, D.; Dusthackeer, V.A.; Rode, H.B. Eur.J.Med.Chem. 2019, 164, 665-677.

15. Kumar, G.; Krishna, V. S.; Sriram, D. Jachak, S. M. Eur. J. Med. Chem. 2018, 156, 871-884.

16. (a) Sastry, G.M.; Adzhigirey, M.; Day, T.; Annabhimoju, R.; Sherman, W. J. Comput. Aid. Mol. Des. 2013, 27, 221-234. (b) Schrödinger Release 2019-3: LigPrep, Schrödinger, LLC, New York, NY, 2019. (c) Friesner, R. A.; Banks, J. L.; Murphy, R. B.; Halgren, T. A.; Klicic, J. J.; Mainz, D. T.; Repasky, M. P.; Knoll, E. H.; Shaw, D. E.; Shelley, M.; Perry, J. K.; Francis, P.; Shenkin, P. S. J. Med. Chem. 2004, 47, 1739 1749. 